Biodiversity Conservation Governance on the Tibetan Plateau:

Cross-scale Linkages and Bridging Organizations

by

Katharina Schlick

\begin{abstract}
A thesis submitted to the School of Geography,
Environment and Earth Sciences,

Victoria University of Wellington, New Zealand
\end{abstract}

In partial fulfilment of the degree of Master of Science in Development Studies

Victoria University of Wellington

[March, 2011] 


\title{
Biodiversity Conservation Governance on the Tibetan Plateau: \\ Cross-scale linkages and bridging organizations
}

\begin{abstract}
The 'roof of the world' is one of the most ecologically diverse and vulnerable regions on earth. Tibetan pastoralists have developed an institutionalized system of checks and balances to regulate access to and conservation of natural resources. However, traditional resource management institutions are greatly transformed by government driven development and conservation projects. Recent studies on adaptive co-management have emphasized the importance of linking institutional and organizational structures at different scales for sustaining socio-ecological resilience and managing cross-scale problems of conservation. Extraordinary conservation accomplishments have been made in cases where government and local communities engage in partnerships for collaborative conservation management.

The methodology for this study incorporated a social network approach that presents a unique analysis of the role of non-governmental organizations (NGOs) in enabling more collaborative conservation governance arrangements in the Tibetan Plateau region. The study is based on a field research in China where I used semi-structured interviews to investigate NGOs perspectives about the main triggers and barriers to more collaborative conservation approaches on the Tibetan Plateau and their role in facilitating the communication among different stakeholder groups. My main findings are that NGOs have significant influence on enabling more collaborative conservation management initiatives. The creation of crossinstitutional partnerships, flexibility in conservation approaches, mutual learning, and trust building processes are seen as the most effective means to more collaborative conservation approaches. Conversely, unequal power relationships, different understandings of how to approach conservation issues and a diversity of partially conflicting interests and priorities are identified as the main barriers.
\end{abstract}

Keywords: Adaptive governance, co-management, multi-stakeholder relationships, NGOs, China 


\section{Acknowledgements}

I would like to thank many people for their help and support during my studies at Victoria University.

First, I would like to thank my supervisor Dr. Michael Gavin for assisting me during the ups and downs of my thesis. His constant encouragement, guidance and critical feedback have been of extreme value for this research.

I am especially grateful to all the interview participants in this research not only for the information you provided to me, but also for the true interest you showed in my research. Without your support, my research would not have been possible.

I would like to thank all my lecturers at Victoria University for their inspirations and academic guidance. Being a student is as much about learning as about making a lifetime experience. Many thanks also go to my friends at Victoria University and in Wellington for sharing their opinions, laughs and worries with me. You made New Zealand a second home to me.

Special thanks go to my parents who even though being far away, always supported me and encouraged me to do what I love. Habibi, I thank you for all your love and support.

Finally, my gratitude goes to the people in China and on the Tibetan Plateau for welcoming me with generous hospitality and friendliness.

This thesis is dedicated to the people of Yushu.

Despite having lost so much through the earthquake, you showed me how important it is to always look ahead in life. 


\section{$\underline{\text { Table of Contents }}$}

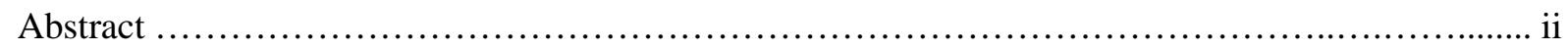

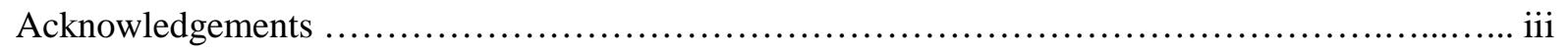

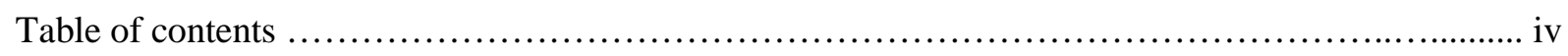

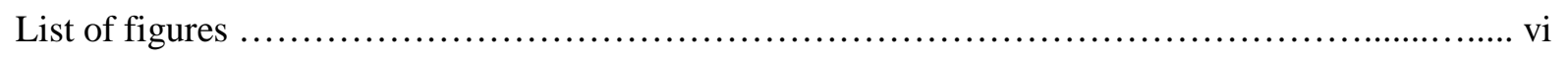

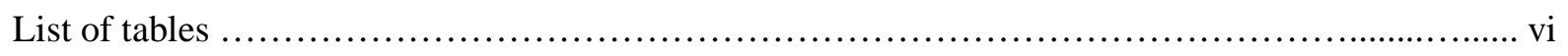

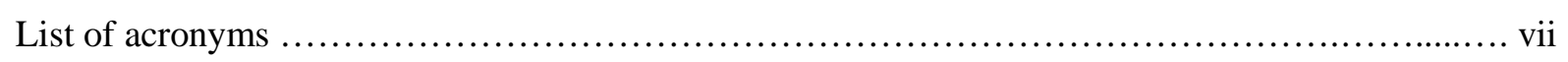

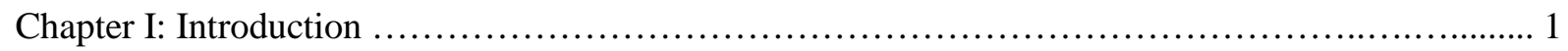

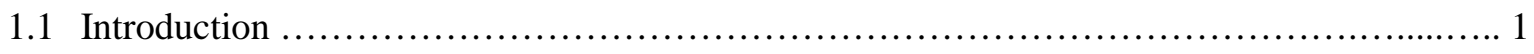

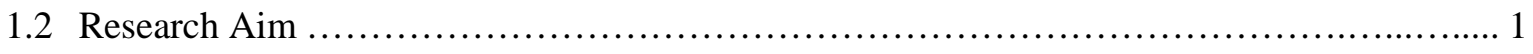

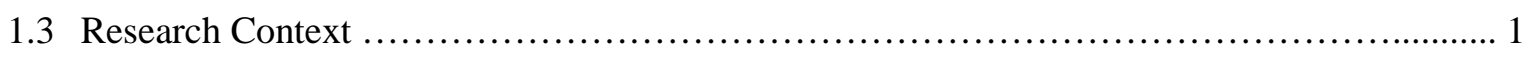

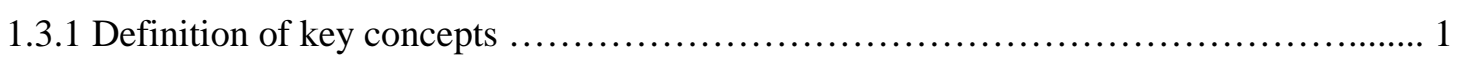

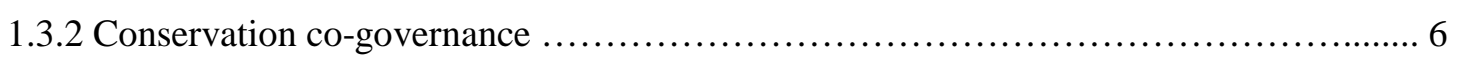

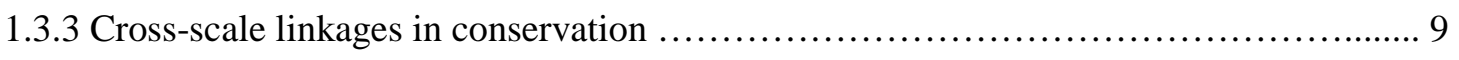

1.3.4 Socio-ecological resilience and adaptive governance ............................ 10

1.3.5 Social networks and the role of bridging organizations in conservation governance.... 13

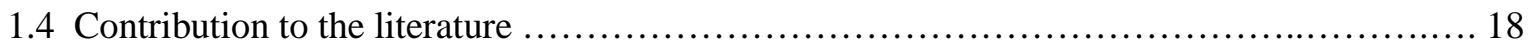

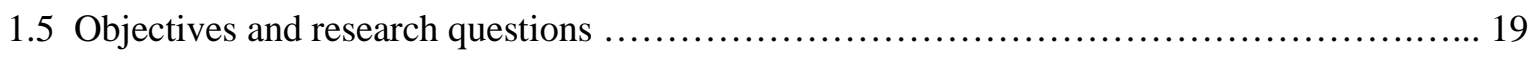

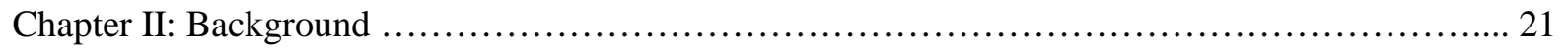

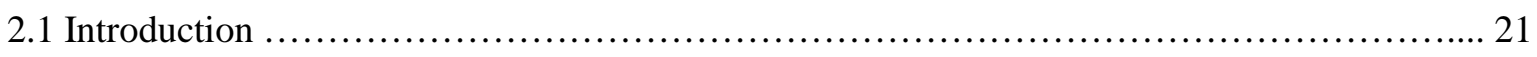

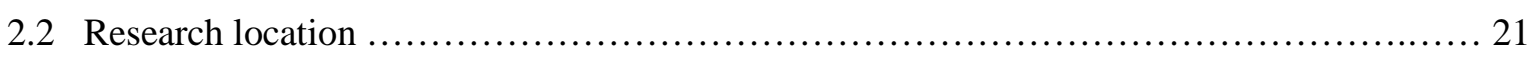

2.2.1 Natural geographic characteristics ....................................... 21

2.2.2 Socio-demographic characteristics and Tibetan Nomad Culture ................... 23

2.2.3 Socio-cultural change and regional developments ............................... 26

2.3 Biodiversity conservation in China and the Tibetan Plateau region ......................... 30

2.3.1 State institutions for biodiversity conservation ................................... 30

2.3.2 Biodiversity conservation on the Tibetan Plateau ................................ 32

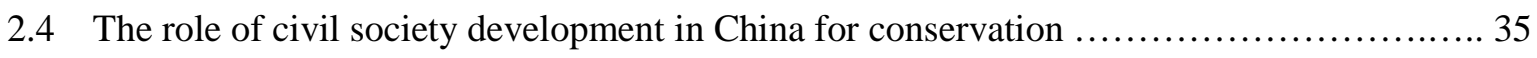

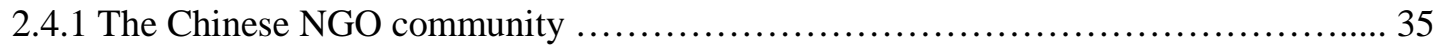

2.4.2 Potential influence of NGOs on conservation governance ........................ 37

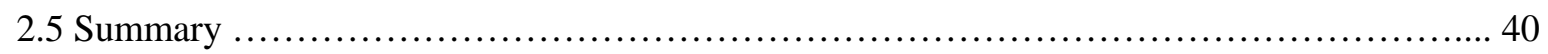

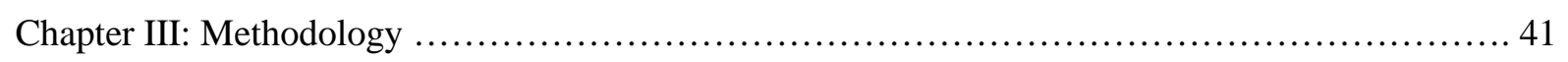

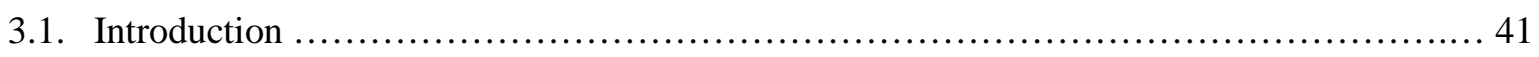

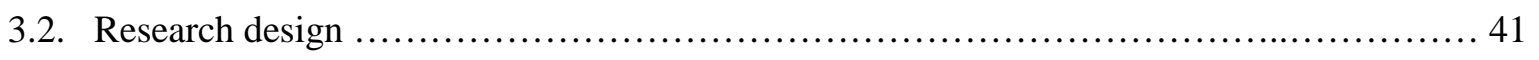

3.2.1 Site selection for case study ........................................... 41

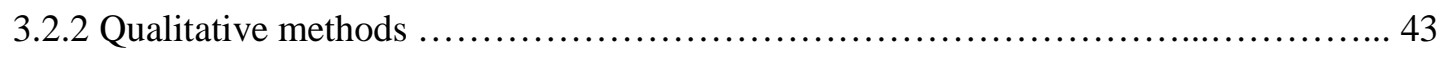

Review of academic literature .............................................. 43 


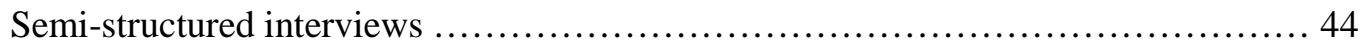

Selection of NGOs to interview ............................................... 46

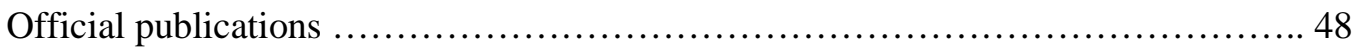

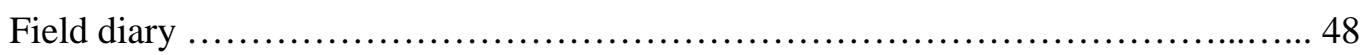

3.3 Ethical considerations .............................................................. 49

3.4. Data Analysis ........................................................................ 50

3.4.1 Pattern coding .............................................................. 50

3.4.2 Micro-analysis of social networks ........................................ 51

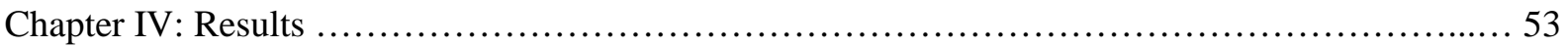

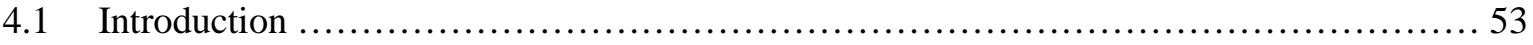

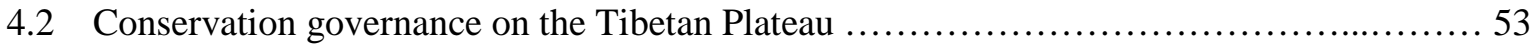

4.2.1 Government approaches towards conservation and NGOs' responses ............... 54

4.2.2 NGO approaches towards negotiating conservation governance arrangements ........ 59

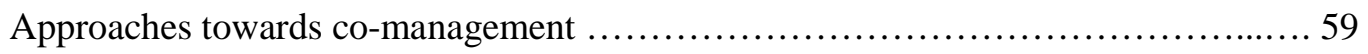

Approaches towards traditional community institutions ...........................6 66

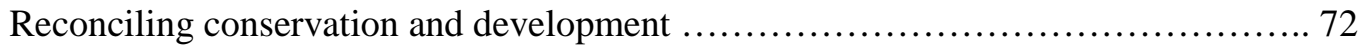

4.3 Conservation networks and stakeholder communication ............................... 76

4.3.1 Cooperation between government and NGOs ................................. 76

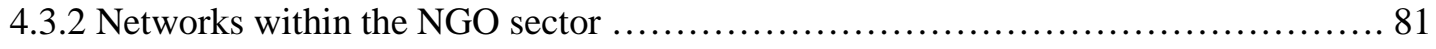

4.3.3 Cooperation between NGOs and local communities .......................... 85

4.3.4 Financial dimensions of conservation and relationships with the donor society ..... 90

4.3.5 Summary of key triggers and barriers...................................... 95

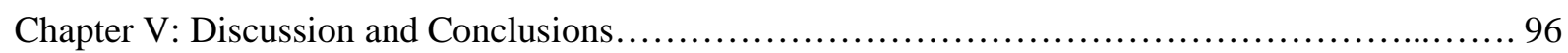

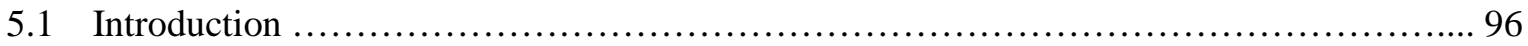

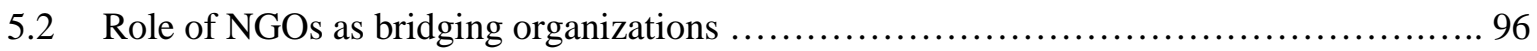

5.2.1 Conservation governance arrangements on the Tibetan Plateau .................... 97

5.2.2 NGOs' role in negotiating conservation governance ............................. 98

5.2.3 Key triggers and barriers to collaborative conservation partnerships ................ 101

5.3 Research limitations and suggestions for future research ............................ 106

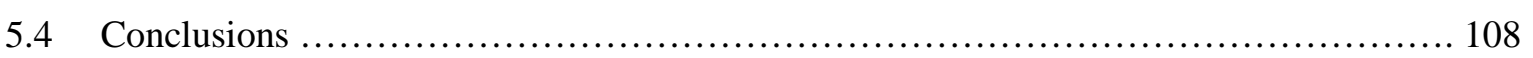

Appendix 1: Key triggers and barriers to collaborative conservation approaches ................. 111

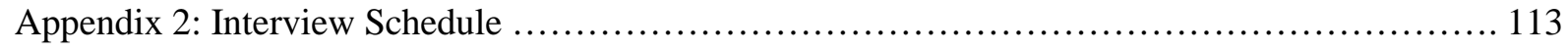

Appendix 3: Human Ethics Approval ............................................... 114

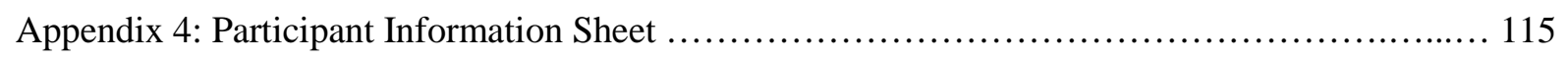

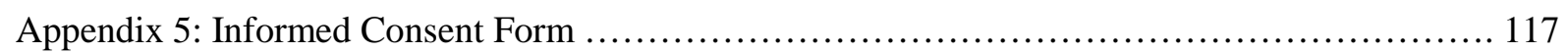

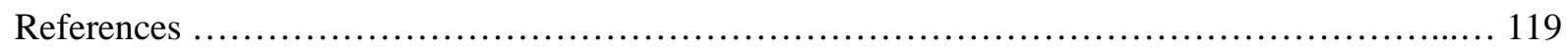




\section{List of Figures}

Figure 1.1: The possible roles of bridging organizations in co-management ................ 15

Figure 1.2: Bridging organizations and constituent networks ........................ 17

Figure 2.1: The location of the Tibetan Plateau ........................................ 22

Figure 3.1: Tibetan Plateau and adjoining areas terrain map ......................... 42

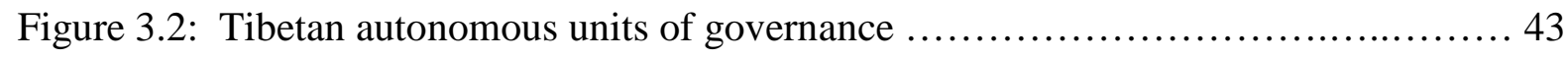

\section{List of Tables}

Table 2.1 Comparison of resource tenure management arrangements $\ldots \ldots \ldots \ldots \ldots \ldots \ldots . \ldots 27$

Table 3.1 List of interview participants .................................... 47 


\section{List of Acronyms}

$\begin{array}{ll}\text { CANGO } & \text { China Association for NGO Cooperation } \\ \text { CCP } & \text { Chinese Communist Party } \\ \text { CERS } & \text { China Exploration and Research Society } \\ \text { EPB } & \text { Environmental Protection Bureau } \\ \text { FFI } & \text { Fauna \& Flora International } \\ \text { FRCD } & \text { Friend of Rural Community Development } \\ \text { GONGO } & \text { Government organized non-governmental organization } \\ \text { MEP } & \text { Ministry of Environmental Protection of the People's Republic of China } \\ \text { NGO } & \text { Non-governmental organization } \\ \text { PRC } & \text { People's Republic of China } \\ \text { QNNP } & \text { Qomolangma National Nature Preserve } \\ \text { SFA } & \text { State Forestry Administration of the People's Republic of China } \\ \text { SFB } & \text { State Forestry Bureau } \\ \text { SNNR } & \text { Sanjiangyuan National Nature Reserve } \\ \text { TAP } & \text { Tibetan Autonomous Prefecture } \\ \text { TAR } & \text { Tibetan Autonomous Region } \\ \text { UYO } & \text { Upper Yangtze Organisation }\end{array}$




\section{Chapter I: Introduction}

\section{$\underline{1.1 \text { Introduction }}$}

This chapter introduces my research aim, followed by a summary of the literature on the major theoretical background to my research. In particular the underlying concepts of collaborative (co-) management of natural resources, cross-scale linkages in conservation and adaptive governance are established. After discussing these concepts, particular attention is drawn to the role of bridging organizations in the governance of biodiversity conservation. The chapter concludes by outlining the contribution of my research to the literature. The research objectives and major research questions are then presented.

\section{$\underline{1.2 \text { Research Aim }}$}

The theoretical purpose of this study is to investigate the relationship between the network position of non-governmental organizations (NGOs) and their influence on institutional transformation of local conservation regimes on the Tibetan Plateau towards more cooperative approaches. There are multiple institutions of control behind biodiversity conservation governance on the Tibetan Plateau. In addition, a range of conservation scenarios from topdown to bottom up can be found in the region. By focusing on the role of NGOs in the conservation process, I will examine how strategic public-private synergies affect the relationship between different stakeholder groups. Through looking at the organizational and institutional dynamics behind conservation governance in the Tibetan Plateau, the research will also provide information on the opportunities and challenges that lie ahead for the development of long-term conservation strategies in cooperation with local communities in the wider regional context.

\subsection{Research Context}

\subsubsection{Definition of key concepts}

Before I will move forward with the major underlying theories of my research, I will define the key concepts involved in the discussion of conservation governance. These include a definition of biodiversity conservation, governance, good governance processes and the concept of co-management. 


\section{Biodiversity and its conservation}

The loss of biological diversity is a problem of global significance that has been widely acknowledged by nation states and civil society. Several multilateral conservation agreements have been signed and revised and an immense amount of money has been channelled to address this ecological crisis. To call global attention to the urgency of the problem, 2010 was declared by the United Nations to be the International Year of Biodiversity. Biodiversity is generally understood as the diversity of living organisms, the genes that they contain and the ecosystems in which they exist (Common \& Stagl, 2005). Conservation seeks to protect the various components of biodiversity for the future.

Increasing environmental problems such as the loss of biodiversity triggered a search for new ways of relating to nature by shifting the focus on the complex interactions and feedback mechanisms between social and ecological systems (Liu et al., 2007; Rammel et al., 2007). Biodiversity plays a substantial role for people through the provision of diverse ecosystem services. At the same time, humans have a considerable impact on biodiversity. Consequently, conservation is as well a social and political process, and therefore not only requires a course of action based on ecological criteria but also an assessment of social, cultural and political institutions (Barrett et al., 2001; Brechin et al., 2002).

\section{Conservation and governance}

A major challenge of the global environment is the challenge of effective governance (Speth \& Haas, 2006). Policy decisions must deal with environmental problems in a way that respects the preservation of both the physical and social constructs for sustainable development. Many problems related to natural resource management can be associated with governance failures, including centralized decision making with insufficient participation of civil society (Pahl-Wostl, 2009). To guarantee ecological security and human well-being there is an urgent need to design institutions that safeguard the dynamic capacity of ecosystem services and that deal with complex governance systems (Common \& Stagl, 2005; Ostrom, 2005).

The literature provides a range of different approaches to conceptualize governance related to natural resources. First of all, the distinction between resource management and governance relating to conservation is sometimes not very clear. Resource management refers to the activities of analysing, developing, implementing and monitoring measures to keep the 
state of a resource within desirable bounds (Pahl-Wostl, 2009). The concept of governance refers in general to arrangements for decision making and power sharing (Brechin et al., 2002). It describes the interaction of different actors and networks that help formulate and implement conservation policy and/or policy instruments and the exercise of power, formally expressed through institutions and articulated by norms (Common \& Stagl, 2005; Fennel et al., 2008).

Institutions are understood as the human devised rules, norms, and strategies used by a set of individuals to organize repetitive activities that produce outcomes affecting those individuals and others that govern human interaction (North, 1990; Ostrom, 1990). Scholars distinguish between regulative, normative, and cultural-cognitive institutions (Scott, 2001: Pahl-Wostl, 2009). Regulative institutions are understood as what is formally allowed by legal structures or regulatory frameworks. Change of regulative institutions is based on negotiation and agreements. Normative institutions can be described informal societal norms, shared but not codified rules of what is right and what is wrong. Cultural-cognitive institutions can be identified with paradigms that strongly influence system understanding of what is thinkable and what is unthinkable (Pahl-Wostl, 2009). Change of normative and cultural-cognitive institutions is not negotiated and occurs through a gradual process.

Treibl et al. (2005) differentiate the major forms of governance according to whether governance is seen as belonging primarily to the realms of politics, polity or policy. The political dimensions of governance calls attention to the way of policy making, how different preferences are translated to effective policy choices and different interests are transformed to unitary action. Governance approaches that are more related to the polity dimension consider governance as a system of rules and institutions that form the action of actors (Ostrom, 2005). Finally, governance may also be defined as modes of political steering and refer thus primarily to the policy dimension (Pahl-Wostl, 2009).

Most fundamentally, governance is a means to an end, not an end in itself. The concept of 'governance' takes into account the increasing importance of modes of governing. Kooiman (2003) differentiates three models of governance: hierarchical governance characterized by state intervention, self-governance, and co-governance consisting of collaboration and interaction among different actors. Consequently, the concept of governance looks beyond the government as the single decision making authority and gives attention to partnerships between actors from public, private and civil society (Common \& Stagl, 2005; Berkes, 2009). However, the actions of other actors in the network are often part of the problem (Brown, 
2001). The literature suggests that both social conflict among a diverse group of participants and environmentally harmful practices are minimized in cases that feature strong governance institutions, strong self-enforcement mechanisms, strong local organizations, and a supportive policy environment (Brechin et al., 2002).

\section{Good governance processes}

Good governance processes are characterized by participation, representation, empowerment, social justice, and organizational features such as being multilayered and polycentric where non-state and private corporate actors participate in the formulation and implementation of public policy or policy instruments that coexist with existing government policy processes (Rhodes, 1997; Fennel et al., 2008). The past decades have seen a shift of authority away from the central state and more attention has been given to the multi-level nature of governance regimes and governance processes (Pahl-Wostl, 2009). In polycentric systems, citizens are able to organize not just one but multiple governing authorities at different scales. Without one central authority dominating all of the other units, polycentric systems are complex, adaptive systems that can generate considerable conflict among the multiple levels as part of the dynamics in social and natural systems (Ostrom, 2005). However, a certain degree of conflict can also help to generate more information about the resource problem and may even be necessary before the state and other stakeholders are willing to enter into negotiations for collaboration on conservation issues (Castro \& Nielsen, 2001; Ostrom, 2005).

While investigating social and political processes related to governance, it has to be questioned how decision making authority is distributed among different stakeholders, how decisions are made, who participates in it, and in what form (Brechin et al., 2002). Good governance processes further distinguish themselves by the degree of local community involvement in natural resource management. Good governance must empower marginalized groups to ensure equitable access to decision making about natural resources. The rationale behind this argument is that people whose livelihoods are affected by management decisions should have a say in how those decisions are made (Berkes, 2009).

However, the concept of community in conservation is vague and leaves room for debate. Agrawal \& Gibson (1999, p.636) argue that greater attention needs to be paid to three critical aspects to be more accurate in depicting communities and their relationship with their natural environment: the multiple actors with multiple interests that make up communities, the 
processes through which these actors interrelate, and, especially, the institutional arrangements that structure their interactions.

There are considerable arguments in common property research that communities may be more appropriate and sustainable alternatives to state and private rule-making and management of natural resources (Agrawal \& Gibson, 1999; Ostrom, 2005). Ostrom (1990) shows that users of a common pool resource ${ }^{1}$ have the capacity for self-organization and selfgovernance and that there are many different viable combinations between public and private. Smaller-scale, self-organized resource governance systems are built on a social system that includes property rights, land and resource tenure systems, and the diverse systems of knowledge pertinent to the natural environment and resources, world views and ethics (Ostrom, 1990; Berkes \& Folke, 2000; Castro \& Nielsen, 2001).

\section{Co-management of natural resources}

Many natural systems are too complex to be governed effectively by a single agency (Berkes, 2009). Co-management has the underlying rationale that by involving different actors in the governing process, the socio-ecological complexities that surround natural resource management can be more adequately addressed (Fennel et al., 2008). The definition of co-management itself varies both in the literature and in practice (Berkse, 2009) ${ }^{2}$. However, it is in general conceptualized as a joint decision making process among the state and local resource users or other key stakeholders about one or more aspects of natural resource access or use (Castro \& Nielsen, 2001, p.230). Central to the idea of co-management is the sharing of both power and responsibility across organizational levels (Plummer \& Fitzgibbon, 2004; Carlsson \& Berkes, 2005). Since there are often multiple local and external interests and multiple government agencies at play, co-management in conservation is as much about managing complex relationships as it is about managing resources (Berkes, 2003). In this context, co-management is a dynamic network activity that is constantly constructed and rebuilt (Carlsson \& Berkes, 2005; Ostrom 2007; Berkes, 2009).

\footnotetext{
${ }^{1}$ Common pool resources refer to "a natural or man-made resource system that is sufficiently large as to make it costly (but not impossible) to exclude potential beneficiaries from obtaining benefits from its use" (Ostrom 1990, p. 30).

2 According to Berkes' (2009) definition, co-management can be understood as power sharing, institution building, trust and social capital, process, problem solving and as governance
} 


\subsubsection{Conservation co-governance}

Collaboration and multi-stakeholder partnerships have increasingly become central components in natural resource management approaches throughout the world (Prager, 2010). Co-management agreements between different stakeholders provide a range of benefits for conservation. Carlsson \& Berkes (2005) point out the benefits of co-management for dealing with natural resource conflicts and define co-management as a continuous problem-solving process. In countries such as China where state politics play a key role in the process of biodiversity conservation, the introduction of more collaborative conservation models can provide more transparency and accountability to the governance of environmental resources (Vernooy et al., 2006). A further justification for co-management is the assumption that increased stakeholder participation will enhance the efficiency and possibly the equity of the linked common pool resource management and social systems (Castro \& Nielsen, 2001). It is argued that communities have incentives to use resources unsustainably when they are not involved in resource management and if their development interests are marginalized (Rao et al., 2003). If communities are involved in conservation, the benefits they receive will create incentives for them to actively engage in sustainable resource management (Agrawal \& Gibson, 1999).

Co-management can also be considered a knowledge partnership. Co-management offers major opportunities for incorporating local knowledge, skills, and practices into formal resource management regimes. The different levels of organization, from local to international, have comparative advantages in the generation and mobilization of knowledge acquired at different scales (Berkes, 2009). Thus, including a broader set of stakeholders facilitates access to different knowledge systems which may be essential for a full evaluation of a resource governance problem and for finding innovative solutions to deal with it (Berkes \& Folke, 2002). Local knowledge for example, provides information on how the resource system may respond to changes in actions of appropriators.

It is argued that systems that are composed by single administrative units and that are based on unilateral decision-making processes are more vulnerable than polycentric arrangements and redundancy (Low et al., 2003). The establishment of parallel systems of rule making, interpretation, and enforcement reduces the probability of failure (Carlsson \& Berkes, 2005). In this sense, co-management is a risk sharing strategy. Further, comanagement is supposed to have lower enforcement costs as local resource users bear the 
costs of monitoring and create legitimate rules to increases local rule conformance (Carlsson \& Berkes, 2005).

On the other hand, a highly decentralized system also bears its limits such as a limited access to scientific information or the inability to cope with larger-scale common pool resources (Ostrom, 2005). Increasing trends in conservation governance towards decentralization and economic liberalization are shrinking the authority and the budgets of authorities (Castro \& Nielsen, 2001). Understaffed and underfunded authorities are overwhelmed by their lack of resources, unable to provide adequate logistical support for basic conservation activities (Castro \& Nielsen, 2001).

However, co-management has been criticized for the degree to which power sharing is actually achieved. Implications of participatory methods depend on if local participation is perceived as an end or a means. Participation as an end in natural resource governance focuses on strengthening the capacity of local resource users and empowers them in decisionmaking processes. By contrast, participation as a means, gives attention to the active involvement of local people in the conservation management process (Carlsson \& Berkes, 2005).Participation as a means is more politically neutral and leaves traditional top-down relations untouched (Parfitt, 2004). Castro \& Nielsen (2001) criticize those that apply comanagement to any situation where local people or other stakeholders engage in partnership, though not necessarily in power sharing with the state. In practice the result may further marginalize communities and resource users and strengthen the state's control over resource policy, management, and allocation (Castro \& Nielsen, 2001). Thus, it is argued that powersharing should be the result and not the starting point of the co-management process (Carlsson \& Berkes, 2005).

While it is generally understood that community participation can play a vital role for conservation (Brosius et al., 2005), participatory methods can also be a source of (new) tensions among different stakeholders (Castro \& Nielsen, 2001; Parfitt, 2004). If the local system is dominated by a local power elite, participatory approaches can be a source of local political dispute. Therefore, multiple voices must be encouraged to expose the power relations in the participatory management process (Ellen, 2002). To increase the capacity for organizational coordination and collaboration, project designers and other practitioners have to take into account questions of structure, performance, culture, and commitment at various organizational levels (Brechin et al., 2002, p.55). 
Co-management agreements should bring about numerous benefits to the stakeholders to get people to negotiate, to reach consensus, and to continue participating (Castro \& Nielsen, 2001). Besides being criticized for its achievements in empowerment of marginalized groups, co-management is said to have a weak impact on the reduction of poverty among resource users (Berkes, 2009). The appropriate design of conservation interventions depends heavily on the biophysical and socio-economic context of a particular setting (Gjertsen \& Barrett, 2004). As many of the earth's biodiversity hotspots overlap with the world's poorest regions, the reconciliation of conservation and development objectives to balance the needs of wildlife populations and human communities are considered to be a main challenge for conservation in developing countries (Fisher et al., 2008, Brosius et al., 2005).

A justification of conservation approaches should not only be focussed on an ecological and economic scale but must also address different ethical imperatives for biodiversity conservation expressed in a respectful attitude towards indigenous people and their traditional resource management institutions (Berkes, 2008). For this reason, the extent to which local knowledge and expertise are valued, and the stability of norms, values, and worldviews must also be carefully examined (Kapoor, 2002; Armitage, 2007). However, the significance and legitimacy of local interests and expertise in natural resource management is a highly polarizing issue that often depends on wider structures of power and the political ecology of the region (Waddell \& Connell, 2007). Even if participatory approaches have become a major pillar in environmental resources management (Pahl-Wostl, 2009), there are many global conservation case studies where the government has delegated little, if any, decision making authority to local communities (Barrett et al., 2001; Zimmerer, 2006).

Several studies suggest that joint management and governance processes are often difficult, if not impossible, to achieve in practice (e.g. Ostrom,1990; Hahn et al., 2006). Therefore, to address contemporary natural resource problems, it is imperative to better understand how barriers to more collaboration can be overcome. Seeing that co-management is understood as a system of relationships and networks (Fennel et al., 2008), the sustainability of projects depends on creating and sustaining linkages with stakeholders at different organizational levels (Brown, 2001). In addition, it is argued that the process of analysing cross-scale linkages can eliminate the power element which is embedded in network relations of people as both individuals and groups (Ellen, 2002). 


\subsubsection{Cross-scale linkages in conservation}

Biodiversity conservation is a complex process that requires multi-lateral action by a diverse range of stakeholders. The need for a better understanding of cross-scale interactions across different levels of organization in natural resource governance is put forward by many scholars (e.g. Berkes, 2008; Ostrom, 2005). Cross-scale linkages are conceptualized as institutional arrangements, formed through the interacting stakeholder groups, which link different levels of governance horizontally across space and vertically across levels of organization (Steinberg, 2001; Berkes, 2003). Cross-scale linkages traditionally did not receive enough attention from common-pool resource scholars because of the field's historic focus on the study of local level institutions (Pahl-Wostl, 2009).

Different scales have different capacities and provide different functions in the system. However, the different levels of organization are not acting in isolation; they continuously feed back to each other (Bodin \& Crona, 2009). The literature argues that cross-scale linkages can improve collaborative governance processes by facilitating: (i) the generation, acquisition and exchange of different types of knowledge and information about the systems under management; (ii) mobilization and allocation of key resources for effective governance; (iii) commitment to common rules among actors fostering willingness to engage in monitoring and sanctioning programs; and (iv) conflict resolution processes (e.g. Carlsson \& Berkes, 2005; Hahn et al., 2006; Scholz \& Wang, 2006; Bodin \& Croona, 2009; Berkes, 2009).

Cross-scale interactions can also affect the influence of social networks on governance processes. It is suggested that the presence of local leaders and a more diverse network of cross-scale linkages are more likely to help achieve local autonomy than the presence of local leaders and a less diverse network of cross-scale linkages (Ostrom, 2005; Berkes, 2009). Local resource extractors are increasingly linked to global networks (large scale). However, it is the structure of the local social networks (small scale) that largely determines who gets to participate and under what conditions (Bodin \& Crona, 2009).

Berkes (2009) outlines three major themes for adaptive co-management in practice: horizontal interaction among stakeholders, vertical interaction of communities with actors at other levels, and iterative learning. Horizontal interaction for example take place in form of linkages across groups functioning at a similar scale such as regional level NGOs. Horizontal linkages are needed to maximize the long-term effectiveness of improvements generated by projects implemented by external agencies by mobilizing local resources (Barrett et al., 2001; 
Brown, 2001). Vertical linkages across different organizational levels are for instance important for enabling grassroots influences on regional and national policies (Brown, 2001). Important processes connecting levels and improving vertical coordination are when actors from one level (e.g. the local level) participate in decision processes at another level (e.g. national level). As institutions that are produced at one level influences processes at another level, actors may thus become actively involved in the production of the rules that influence them (Pahl-Wostl, 2009).

However, Folke et al. (2005) criticize that the real challenge lies in dealing with systems that are not only cross-scale but also dynamic. In addition, coupled human-natural systems constantly change over time (Liu et al., 2007). Addressing the various challenges related to resource governance is hampered by the lack of understanding processes of change and adaptation in multilevel governance regimes (Young, 2008). For this reason, there is a need for adaptive co-governance processes in which cross-scale linkages and socio-ecological systems are assessed regularly.

\subsubsection{Socio-ecological resilience and adaptive governance}

Governance of ecosystems is inherently difficult since most environmental problems that matter to society involve complex and dynamic human organizations and ecological relationships (Berkes et al., 2003; Liu et al., 2007). The ability of governance systems to deal with uncertainty and surprise is an essential requirement for their sustainability in times of increasing uncertainty due to environmental change (Pahl-Wostl, 2009). To protect biodiversity, conservation requires a reorientation towards the protection of ecosystem functions and safeguarding socio-ecological resilience. Resilience reflects the degree to which a complex adaptive system is capable of self-organization and absorbing perturbations (Folke et al., 2004).

Several studies highlight the importance of context specific knowledge that members of local communities possess and the institutional arrangements they forge to achieve successful, local level resource management (e.g. Berkes et al., 2000; Brosius et al. 2005; Berkes et al., 2006; Shakeroof \& Campell, 2007). Indigenous peoples occupy a substantial share of the world's little disturbed ecosystems and each culture constructs its own standards for socialecological adjustment formulated in traditional resource management institutions (Berkes, 2008). 
Local traditional management systems often embody an adaptive management approach in which they emphasize feedback learning and address uncertainty (Berkes et al., 2003). Communities in mountain environments and high altitudes as they can be found on the Tibetan Plateau rely on biodiversity to maintain subsistence livelihoods and resilient farming systems under some of the most difficult conditions on earth (Rao et al., 2003). Agriculture under these environmental conditions has to cope with a high degree of inaccessibility, fragility, marginality, diversity, and unique production opportunities and thus requires a high degree of human adaptation to local environments based on collective leadership (Jodha, 2000). It is assumed that government agencies are unlikely to be familiar with the characteristics of local resource systems and research shows that top-down centralized management is poorly suited for specific local conservation contexts (Ostrom, 1990; Berkes $\&$ Folke, 1998;). For this reason, natural resource management would benefit if actors agree on common rules and practices, coordinating usage, engaging in conflict resolution, negotiating various tradeoffs, sharing information, and building common knowledge (Folke et al., 2005; Bodin \& Crona, 2008).

The ecological knowledge of resource-user groups is of high relevance in the context of socio-ecological resilience and co-management. But even remote environments nowadays face a rapid decline of their traditional resource management systems through internal and external driving forces. Traditional practices persist alongside government conservation initiatives but often, local capacities and strategies are not capable of coping quickly enough with rapid change and innovating institutions to suit contemporary requirements (Jodha, 2000). The innovation process needs policy support and technical backing.

Adaptive governance and social learning have been identified as essential for governing social-ecological systems during periods of abrupt change (Folke et al., 2005; Pahl-Wostl, 2009). Adaptive governance of ecosystems generally involves polycentric institutional arrangements operating at multiple scales with explicit focus on the adaptability of the joint management process in response to environmental and social change (McGinnis, 2000; Ostrom, 2005). Adaptive governance systems often self-organize as social networks with actor groups that draw on various knowledge systems and experiences for the development of a common understanding and policies (Folke et al., 2005). Janssen et al. (2000) suggest that complex adaptive systems such as nomadic pastoralism can be studied by adaptive agent models that deal with the interaction between policy, pastoralists' decisions and a diverse range of actors. Recognizing that multiple actors exist at the local level is a useful step 
forward because it forces researchers to consider their different and dynamic interests: "Institutions can change because of constant challenges to their form by the actions of individuals whose behaviour they are supposed to influence" (Agrawal \& Gibson, 1999, p.367).

Adaptive co-management is a way to operationalize adaptive governance and a recent expansion of the co-management concept (e.g. Olsson et al., 2004; Armitage et al., 2008; Fennel et al., 2008). The concept of adaptive co-management combines the dynamic learning characteristic of adaptive management with the linkage characteristic of cooperative management (Olsson et al., 2004). It is understood as a process by which institutional arrangements and ecological knowledge are tested and revised in a dynamic, ongoing, selforganized process of learning-by-doing (Folke et al., 2002). Armitage et al. (2008) point out the learning aspect of adaptive co-management as a reaction to social-ecological change, with participants recognizing that new management strategies, rules or procedures are required because resource depletion and livelihood disruptions are likely.

Socio-ecological resilience not only requires an understanding of contemporary patterns of human-environment interactions but also sensitivity to the needs of local communities. Adaptive co-management pays more attention to the needs of resource users than adaptive management, and is more aware of the importance of learning and adapting than comanagement (Berkes, 2009). Thus, the instrumental rationale of adaptive co-management is primarily to solve resource problems through a collaborative process to foster ecologically sustainable livelihoods and secondarily to conserve natural resources (Plummer \& Armitage, 2007; Fennel et al., 2008).

Recent studies on adaptive co-management have emphasized the importance of institutional and organizational structures at different scales for sustaining socio-ecological resilience and managing cross-scale problems of conservation (Olsson et al., 2004, Carlsson $\&$ Berkes, 2005). However, as cross-scale linkages are dynamic in their nature their influences in the linked social-ecological system changes over time. This creates fundamental problems for division of responsibility between centralized and decentralized agents (Folke et al., 2005). An important aspect in this context is the emergence of organizations that have the ability to bridge local actors with other scales of organizations and facilitate coordinated action (Folke et al., 2005; Berkes, 2009). 


\subsubsection{Social networks and the role of bridging organizations in conservation governance}

The relationships among stakeholders in conservation are critical. Recent research has identified the existence of social networks as a common and important attribute in cases where different stakeholders have come together to deal effectively with natural resource problems and dilemmas (e.g. Folke et al.,2005; Hahn et al., 2006; Olsson et al., 2008; Scholz $\&$ Wang, 2006). Social networks can be even more important than the existence of formal institutions for effective enforcement and compliance with environmental regulations (Scholz $\&$ Wang, 2006). To address the critical question which network characteristics and learning processes foster cooperation across groups requires the examination of networks among actors that connect local mobilisations to the larger environmental movement and political systems (O'Rourke, 2004). In this section, I will use social network theory to describe and illustrate bridging organizations as an organizational form. Then, I will outline some key characteristics of bridging organizations and their role in networks. Finally, I will discuss the potential influence of bridging organizations on conservation governance.

\section{Social network theory}

The set of actors and the relationships that connect these actors can be considered a social network; and the network of relationships within which a project is embedded has important consequences for its success or failure (Kilduff \& Tsai, 2003). A structuralist approach towards social network analysis locates actors in the whole field of interdependent forces instead of isolating actors as units of analysis. The emphasis is on how actors connect to others across different scales and on an analysis of the structures that shape action (Burt, 1992). As suggested by the structuralist approach, many individual actors may be constrained by elements in the macro-environment such as government agencies or large organizations (Kilduff \& Tsai, 2003). However, smaller actors are also said to have some ability to influence the structures of constraint within which they operate.

The post-structuralist approach towards social network analysis draws on individual actors' perceptions of their position in the network and how these perceptions drive actions that change networks. The poststructuralist perspective focuses especially on the shifts and changes in subjective interpretations of norms, values and behaviours (Burt, 2004). A poststructuralist approach further puts emphasis on the significance of weak-ties for bridging 
structural holes within a network (i.e. gaps in the flow of information between individual groups within a network; Granovetter, 1973; Burt, 1992). ${ }^{3}$

An actor who sits between many other actors in the network is said to have the potential to bridge structural holes between others in the network. An actor occupies a central position in the network either by having many ties to other actors, being able to reach many other actors, connecting other actors who have no direct connections, or having connections to centrally located actors (Kilduff \& Tsai, 2003). This position grants the actor the ability to influence the flow of resources between others, and it also provides the actor with a diversity of resources provided by the bridging ties (Burt, 2004). Bridging ties (i.e. ties that link otherwise disconnected or loosely connected sets of actors) among different subgroups enhance productivity, collective action, innovative capacity, and facilitate connections with external actors and wider stakeholder groups (Bodin \& Crona, 2009).

It is often equally important to assess how actors can use their position in the network to influence the natural resource governance process. A highly centralized conservation network is only efficient in specific cases (Bodin \& Crona, 2009). If only few ties exist among actors, joint action maybe harder to achieve. Central actors can use their influence to benefit others in the network not only through encouraging but also coordinating collective action. On the other hand, too many ties can foster actor homogenization and reduce the capacity for effective collective action to deal with changing conditions (Bodin \& Crona, 2009). In addition, a central actor that holds many ties to other actors in the network can be subject to diverse, partially conflicting interests (Brown, 2001).

\section{Role of bridging organizations in conservation}

Numerous reasons justify that bridging organizations may play a critical role in comanagement arrangements (see Figure 1.1, p.15). Folke et al. (2005) emphasize that bridging organizations have the ability to strengthen social capital and the capacity for effective governance of multilevel organizations involved in ecosystem management. In addition, it is supposed that bridging organizations reduce the costs of collaboration and conflict resolution,

\footnotetext{
${ }^{3}$ The strength of a tie can be measured as a combination of the amount of time, the emotional intensity, the intimacy and the reciprocal services which characterize the tie. Strong ties are understood as being frequent, long-lasting and effect-laden social relationships, whereas weak ties are infrequent and distant (Granovetter, 1973)
} 
and enable legislation and governmental policies that support self-organization of local communities (Folke et. al., 2005).

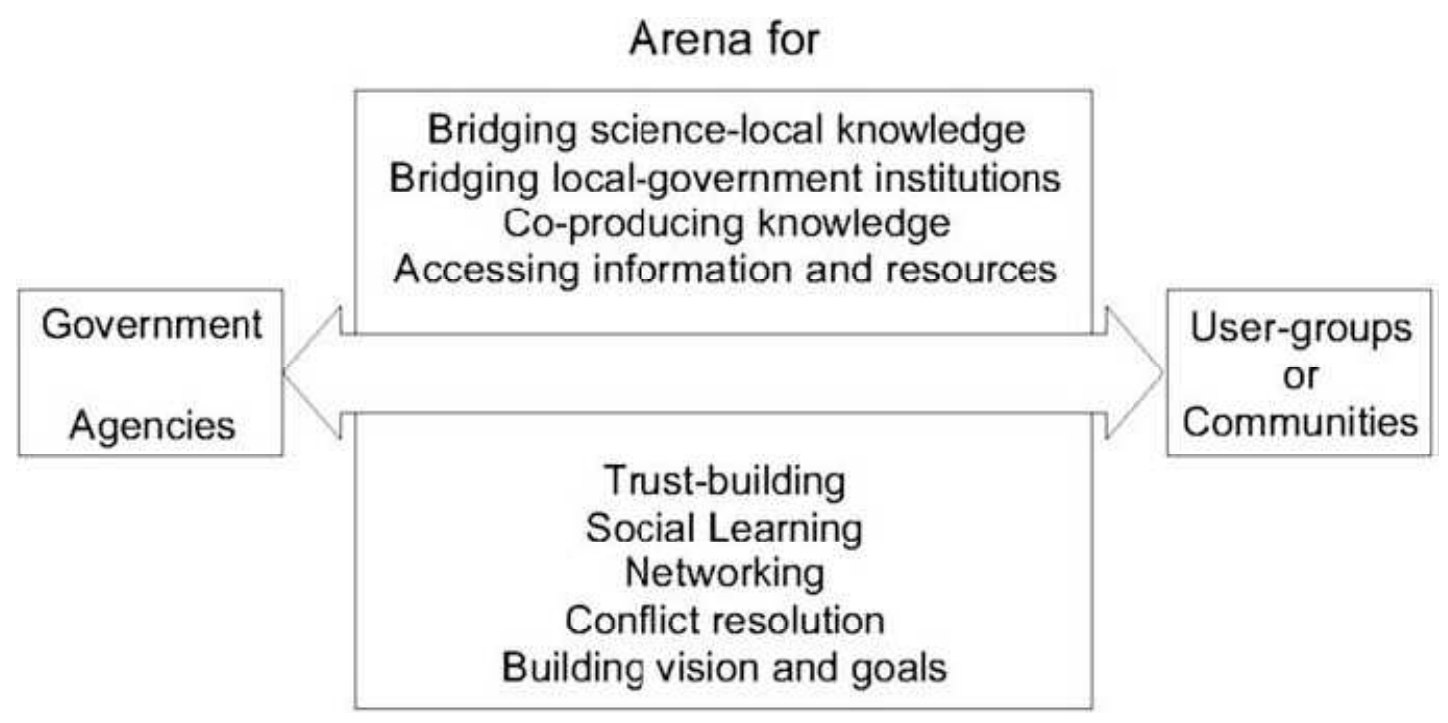

Figure 1.1: The possible roles of bridging organizations in co-management (Adapted from Berkes, 2009, Fig.2, p. 1696).

Brown (2001) argues that bridging organizations are central players in an increasingly multi-sectoral development paradigm and play a critical role in inter-organizational partnerships that enable cooperation on problems that cannot be solved by a single agency. In addition to their functional role in addressing conservation issues, conservation NGOs that act as bridging organizations can make important contributions to rural development by cooperating with development agencies or extending their project activities (Zahler, 2010). Also, bridging organizations sometimes hold together loose associations of organizations that are similar to one another in interests and concerns (Astley \& Fombrun, 1983). By developing tighter and more centralized organizational forms, these coalitions increase their potential in influencing regional or national conservation policies and facilitate social learning (Brown, 2001; Berkes, 2009).

However, bridging organizations face considerable challenges in developing countries such as differences in wealth, power, and culture among the different stakeholder groups (Brown, 2001). In this context, it is an advantage that bridging organizations thrive under open institutions, which provide flexibility and space for dealing with the ambiguity of multiple objectives and which makes them more adaptive to changes in the socio-ecological environment (Folke et al., 2005). As a consequence, bridging organizations serve as catalysts 
and facilitators between different levels of governance, and across resource and knowledge systems (Folke et al., 2005). These are important features for strengthening the adaptive capacity of local actors. International NGOs are especially supposed to have the ability to gain access to high levels of the central government and can act as facilitators between rural and often marginalized communities and central government agencies (Morton, 2008).

While it is accepted that governmental and non-governmental institutions contribute significantly to confronting global environmental changes, such as the loss of biodiversity, the role of local institutions cannot be ignored. Community-level governance structures are often built around the need to manage land. Though, these local governance institutions can be destroyed by diverse factors such as population growth, national and global market forces or global environmental change. Traditional systems of governance are rarely capable of dealing with novel stresses creating a "rural governance gap" (Zahler, 2010). One possible role of conservation NGOs is to build local institutions for the improvement of natural resource management to fill this gap. Bridging organizations bring in new ideas, but more importantly they bring in networks of contacts. These contacts can help local communities to cross the geographic, cultural, and political barriers of the national landscape in ways that would have been hard for the locals to do on their own and gain access to nonlocal institutions and resources (Jamal et al., 2007; Zahler, 2010).

Steinberg (2001) supposed that the more diversely connected activists are at the local, national, and international level, the more they will be able to achieve improvements in local biodiversity governance. By creating linkages to external actors, bridging organizations are able to transfer intellectual expertise from the international to the local level and encourage knowledge co-production and the building of social capital (Berkes, 2009). The networks of exchange themselves are the formal or informal rules that determine the actual command over technical, financial, and other resource endowments (Zahler, 2010). These include access to organizations with technical assistance and financial resources, sources of technology, donors, and alternative trading networks (Folke et al., 2005).

However, being in a central position in a network may not by itself necessarily lead to higher influence (Brodin \& Crona, 2009). On the other hand, an individual still can have significant influence without possessing a central position in the network. The literature shows that the performance of groups in an organizational context is largely influenced by empowerment, decision making processes, conflict resolution strategies, knowledge and skills, experimentation and innovation, and organizational leadership (Ostrom, 2005; Folke et al., 
2005). Figure 1.2 (p.17) demonstrates the advantages and disadvantages of acting as bridging organizations. Through their central position, bridging organizations potentially have influence on diverse stakeholders. At the same time, bridging organizations are subject to the various demands of the different stakeholders and which may lead to diverse, often conflicting interests. Hahn et al. (2006) show by a case study on adaptive co-management of a wetland landscape in Sweden the functional role of bridging organizations in trust-building and conflict resolution. The authors further highlight how the interplay of formal and informal institutions in the co-management process enabled the bridging organization to build social networks between different stakeholder groups (Hahn et al., 2006).

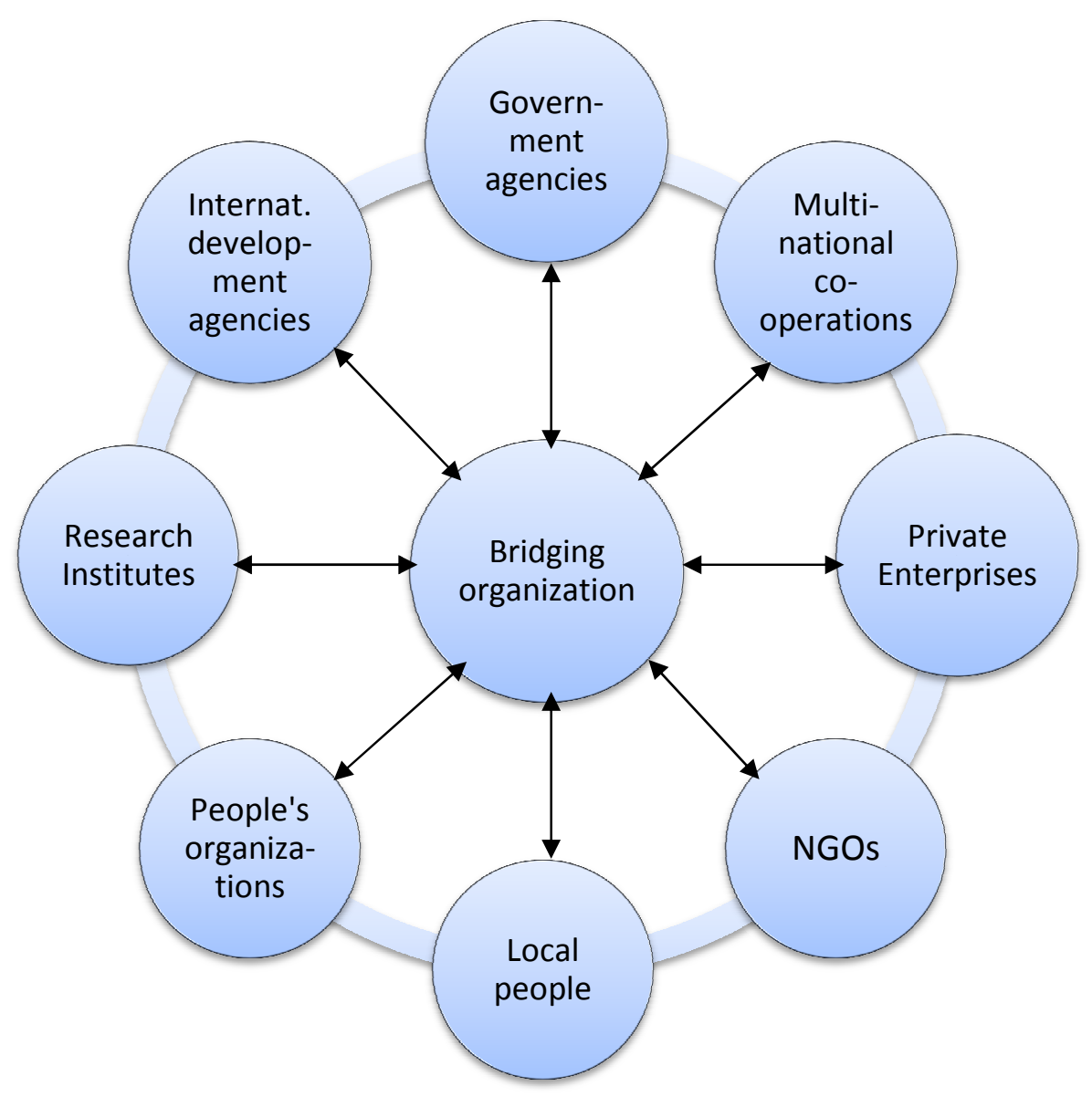

Figure 1.2: Bridging organizations and constituent networks (Adapted from Brown, 2001, Fig. 1, p. 812)

Consensus building and conflict resolution are two important prerequisites for natural resource governance but networks are difficult to establish and maintain in a sensitive 
environment (Ostrom 1990; Rootes, 2008). Conservation projects in a sensitive socio-cultural, political environment have to be aware of the links between environmental struggles of indigenous populations and human rights abuses (Tauli-Corpuz \& Carino, 2004). Escobar (1998) makes a strong case for the impact of social movements and alternative development strategies in political sensitive regions on conservation, empowerment and cultural affirmation. The position among NGOs is varied ideologically, politically and economically. Southern NGOs especially pay more attention to identity politics and are more culturally embedded (Ellen, 2002). Organizations that endeavour to provide support in more politically sensitive sectors, such as human rights or governance reform, often meet with strong cultural or political resistance (Zahler, 2010). Co-management strategies require a significant amount of time to include marginalized groups in decision making processes and build strong local coalitions (Noble, 2000). An advantage of conservation NGOs towards development NGOs is that conservation NGOs work at sites for many years, trying to promote long-term changes and that they are considered to be more politically neutral (Zahler, 2010).

\subsection{Contribution to the literature}

The literature suggests the need to better understand the role that NGOs are playing as representatives of civil society in environmental concerns and in supporting local conservation governance efforts by influencing state and corporate decision making (see e.g. O'Rourke, 2004; Berkes, 2009). Despite an increasing academic interest in the role of NGOs in environmental governance in recent years, few scholars have addressed their function as bridging organizations between different conservation stakeholder groups (see e.g. Wilshusen \& Marguia, 2003; Hahn et al., 2006; Jamal et al., 2007; Berkes, 2009). In particular, the literature contains no detailed case study analyses of how networks help, what makes bridging organizations work, and why redundancy is important (Berkes, 2009). It is argued that a social network perspective holds great potential in enabling analyses of various cross-scale interactions (Bodin \& Crona, 2009). In addition, Bodin \& Crona (2009) suggest that network studies of successful cases can reveal key network characteristics that benefit governance processes.

Until recently, relatively little work has investigated the influence of NGOs on environmental governance in China. And in particular, no study has used a network analytic approach to study the role of NGOs in conservation governance in the Tibetan Plateau region. 
Addressing the increasing environmental challenges that put pressure on the local landscape of the Tibetan Plateau requires adequate governance of the regions natural resources. The growing body of work on local environmental management, land use, climate and ecological change in the Tibetan Plateau testifies the ecological importance of the region (e.g. Banks et al., 2003, Gruschke, 2006, Foggin, 2008; Morton, 2008). However, the institutional dynamics behind biodiversity conservation and cross-scale linkages between different levels of governance are poorly understood and addressed.

My study takes up the theoretical issues related to biodiversity conservation raised above on the background of Chinese conservation policies and forms of local environmental stewardship. By taking the Tibetan Plateau as a case study, this research links the theories of biodiversity conservation governance in China with practical experiences from the viewpoint of NGOs. It not only provides a current contextual picture of how the changing institutional environment is affecting local level institutional arrangements for biodiversity conservation in the Tibetan Plateau region but also exemplifies the challenges and opportunities faced at the different governance levels. It will test out the theory that multiple linkages are a key to conservation success and reveal the problems related to a wider network of stakeholders.

\subsection{Objectives and Research Questions}

Based on the wider context outlined in the previous sections, the following tasks and related research questions will be undertaken as part of this research:

\section{What are the primary forms of conservation governance currently in practice in} the Tibetan Plateau region in China?

What are the major challenges for conservation in the region?

How do government arrangements for conservation shape access to and control of natural resources?

\section{What role do NGOs play in shaping conservation governance arrangements in the} region?

How are governance arrangements constructed, and how might this be recognised so as to open up the possibility of more collaborative arrangements? 
How, if at all, do NGOs connect central levels of decision making to those of the local level?

How are local traditional resource management institutions approached by government authorities and NGOs?

\section{What are the main triggers and barriers to more collaborative approaches?}

How are networks of stakeholders and conservation governance processes shaped at different organizational levels?

Which network characteristics and learning processes foster cooperation across these groups?

What role do NGOs play in facilitating the communication between different conservation stakeholder groups? 


\section{Chapter II: Background}

\section{$\underline{2.1 \text { Introduction }}$}

The previous chapter has outlined the main theoretical background of my research by discussing the concepts of conservation governance, (adaptive) co-management and the role of cross-scale linkages in conservation. This chapter tailors the key theories to the Chinese biodiversity conservation framework and to the regional context of the Tibetan Plateau. I will provide basic geographical and social facts about the research location with particular focus on local traditional natural resource management institutions. Then I will give a brief overview over recent biodiversity conservation developments in China and in the Tibetan Plateau region to identify the major institutional setting for conservation, and then look at the connection between conservation and civil society development in China. The chapter concludes by providing an overview of the major underlying challenges for biodiversity conservation in the research location.

\section{$\underline{\text { 2.2 Research Location - The Tibetan Plateau }}$}

\subsubsection{Natural- geographic characteristics}

China is considered to be a 'mega-diversity' country due to its huge variety of natural geographic conditions. However, biodiversity conservation in China is facing many challenges. On the one hand, China is the world's most populous country and threats to the natural environment are continuously increasing along with a rapidly developing economy. On the other hand, China is surprising the international conservation community through its introduction of massive ecological restoration and conservation projects in form of large-scale protected areas. Especially China's peripheral regions in the western ${ }^{4}$ part of the country are characterized by high degrees of species richness and endemism (McBeath \& Leng, 2006). Even if there are no 'mega-diversity hotspots' in western China and fewer habitats for vertebrates, the region is nevertheless home to a unique biodiversity of global importance that also supports its local human inhabitants with wide array of ecological, economic and sociocultural functions (Harris, 2008).

\footnotetext{
${ }^{4}$ Western China is rather a political than geographical term. It describes the natural geographic, social and economic differences and inequalities between China's central and western provinces and the more developed eastern part of China (Lu \& Neilson, 2004).
} 
With an average elevation over $4500 \mathrm{~m}$, the Qinghai-Tibet Plateau ${ }^{5}$, often referred to as "the roof of the world", is the largest high altitude landmass on earth. It covers a size of 2.5 million square kilometres and accounts for approximately 25 per cent of China's geographical land area. The Tibetan Plateau is one of the most ecologically diverse landscapes on earth. Although the majority of the Plateau is dominated by high-altitude grasslands, a huge variety of ecosystems can be found in the region, including wetlands, forests, shrublands and mountain ranges (Fleming et al., 2007). The result of this diversity in ecosystems is a unique richness in various endemic and endangered species, including the iconic chiru and kiang (Zhang et al., 2002). With thousands of glaciers scattered across the Plateau and the Himalayas, the region has the most glacially stored freshwater on the planet outside of the Polar Regions. The glacier-fed rivers that originate from the Tibetan Plateau make up the largest river run-off from any single location in the world. The river network plays a significant regional role as it supplies downstream communities in most south-east Asian countries as well as huge parts of China with freshwater.

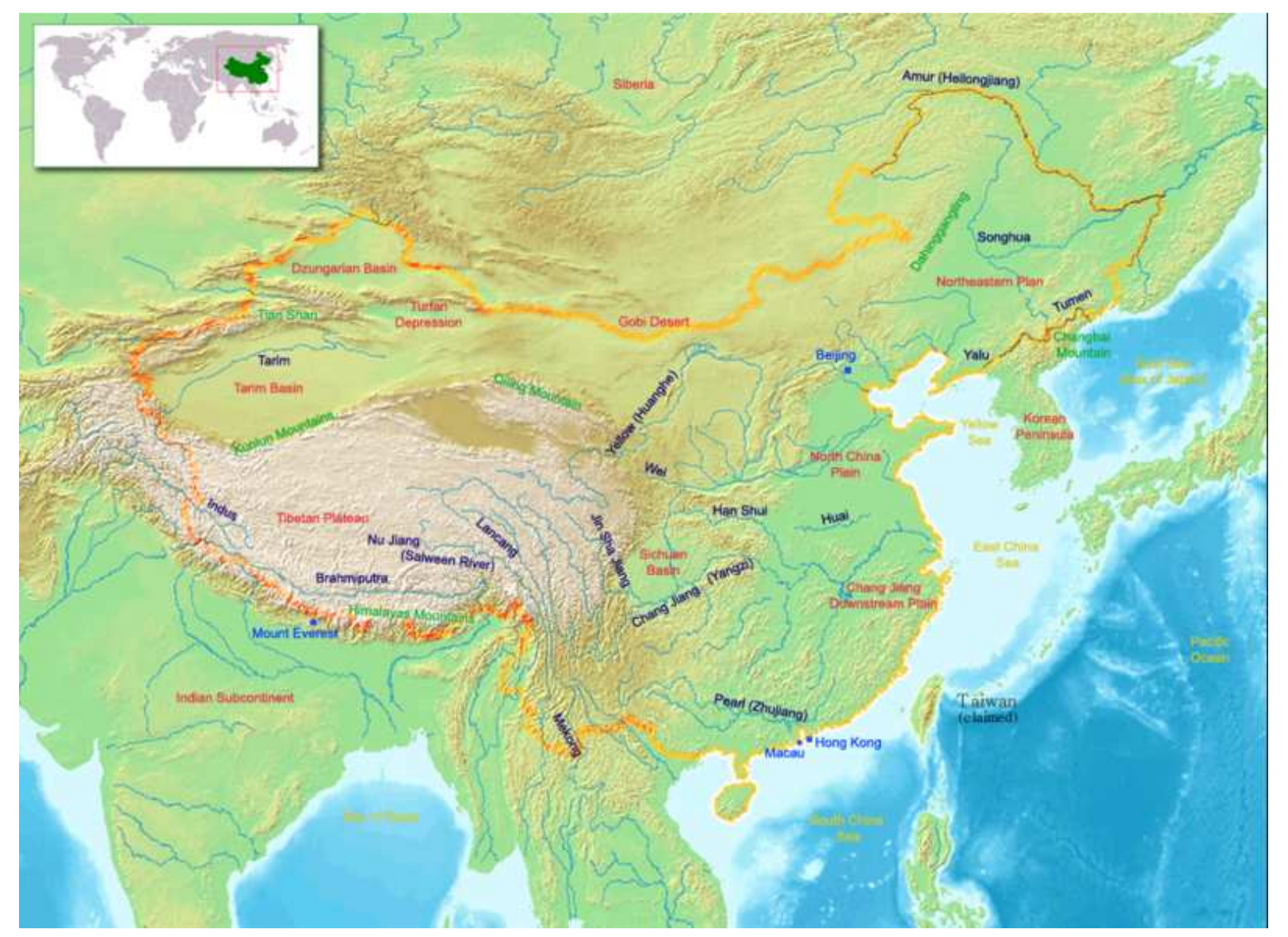

Figure 2.1: The location of the Tibetan Plateau, showing major rivers and mountain ranges (Adapted from http://commons.wikimedia.org)

\footnotetext{
${ }^{5}$ Afterwards referred to as Tibetan Plateau
} 
However, due to its unique geographical location, the environment on the Tibetan Plateau is extremely fragile. Unsustainable resource use in this region can have local, regional and even global repercussions. Recently human- induced environmental problems are rapidly increasing across the Plateau. Yak herding is an integral part of Tibetan culture, however, overgrazing is now threatening the fragile ecosystem in some parts of the region (Harris, 2008). Deforestation, increasing desiccation and soil erosion are changing the face of the landscape (Fleming et al., 2007). In addition, unregulated mining dumps and landfills established to cope with the rising waste from urban centres are threatening the healthy state of the Plateau's ecosystems (Goldstein et al., 2003). Furthermore, another significant environmental problem is the decrease in local Fauna for the most part due to illegal hunting activities for game and wildlife by poachers from outside (Foggin, 2005; Harris, 2008). Finally, growing attention has been given to the impact of climate change on the region's ecosystems. With extensive grassland ecosystems that store carbon in their plants and soil, the Plateau has the potential to modify global carbon cycles and influence climate (Morton, 2008). Despite official recognition of this ecological crisis, there is little information and long-term scientific data on the state of degradation and what degradation means by Chinese definitions (Harris, 2008).

\subsubsection{Socio-demographic characteristics and Tibetan Nomad Culture}

The impacts of regional environmental degradation are greatest on poor communities that are least able to adapt (Morton, 2008). Biodiversity provides a fundamental base for pastoralism and to the overall economic systems. Receiving the majority of their income and nutrition from livestock breeding, the people of the Plateau are highly dependent on ecosystem services. However, they also share a high vulnerability to natural disasters and changes in the ecological environment. Perceptions of people in China's densely populated, fast developing eastern provinces about the country's sparely populated, geographically larger western provinces are rather negative as they often associate it with poverty and backwardness (Harris, 2008). Supporting less than 1 per cent of China's population, the Tibetan Plateau has one of the lowest population densities in the world. The people of the Plateau are primarily of Tibetan ${ }^{6}$ ethnicity. Xining and the northern parts of Qinghai Province are more a mixture of different ethnic groups and eastern and central Sichuan is dominated by

\footnotetext{
${ }^{6}$ Tibet has a large variety of ethnic minorities (Klieger, 2007). With Tibetan minorities I refer to indigenous minority groups belonging to the larger Tibetan ethnicity.
} 
a Han Chinese culture. Rural areas in Qinghai and the western third of Sichuan province by contrast, are strongly dominated by a Tibetan culture and lifestyle with ethnic Han Chinese usually being the 'minority'. Tibetan culture shares diversity in itself and there are many small ethnic Tibetan subgroups on the Plateau that see themselves culturally distinct from the major ethnic classifications of Khampa and Amdo Tibetans (Klieger, 2007). However, an increasing diversification of the socio-cultural landscape can particularly be noticed in urban areas (Kolas \& Thowsen, 2005).

To do conservation in China, it is important to develop an understanding of local culture (Coggins, 2003; Harris, 2008). Differences in attitudes towards nature and its resources amongst different ethnic groups create complex conditions for conservation in China. A number of studies have examined the cultural ecology of Tibetan minority groups (e.g. Xu et al., 2006; Studley, 2007), supported by a few studies on regional nomadic pastoral practices and their relation to ecological change and local conservation responsibilities (see e.g. Miller, 1998; Banks et al., 2003; Sheehy et al., 2006). The Tibetan Plateau has a strong tradition of natural resource stewardship among its minority nationalities. Traditional orientations toward nature and conservation are strongly influenced by Tibetan Buddhism that respects all living beings and considers wild animals as a symbol of freedom (McBeath \& Leng, 2006; Fleming, et al., 2007).

Khampa Tibetans inhabit a large area in the eastern part of the Tibetan Plateau, which overlaps 80 percent of the Mountains of Southwest China hotspot. In traditional Khampa Tibetan cultural perspectives, every Tibetan village and monastery has its designated sacred sites nearby mountains, forests, lakes and rivers. Khampa Tibetans believe in reincarnation and safeguarding the land and the wildlife is part of protecting their past and their future (Studley, 2007). Han Chinese attitudes towards nature by contrast are predominantly utilitarian, seeing wildlife mainly as a resource (Coggins, 2003). On the other hand, consumption of wildlife is an integral part of Tibetan culture as well. Tibetan pastoralists not only largely replaced wild animals with domestic ones, but have specialized in the trade of wild species products and a wide array of wildlife products can be found on Tibetan bazaars (Harris, 2008).

The Tibetan Plateau is characterized by a complex physio-geographic environment, multidimensional topography and slope aspect (Zhang, 2002). Permafrost is widespread and solar radiation intense (Foggin, 2005). The harsh environmental conditions on the Plateau place significant limitations on farming practices. Unlike in the past when people hunted and 
migrated by following wildlife throughout the year, people now manage their own land and herd livestock, mostly sheep and yak (Miller, 2002). The development of nomadic or seminomadic pastoralism is considered one of the greatest advances in local culture. One of the most important cultural characteristics of nomad societies is their close and detailed knowledge of local natural resources (Xu et al., 2006). Nomadic herders are aware of the fragility of their environment and sensitive to any ecological destabilization of it and have adapted to the environmental conditions of the Tibetan Plateau region through a complex adaptive system of land management practices (Sheehy et al., 2006).

Claims that nomadic pastoralism is inherently self-destructive are indifferent to the long term conservation outcomes of traditional pastoralism in the region (Miller, 1998). The common property of extensive pastoralists is an institutionalized system based on checks and balances, overlapping temporal and spatial rights and constraints which regulate access to pastures. Traditionally, natural resources have been managed by institutions created by largely autonomous, self-regulating, tribal communities. Among nomadic pastoralists, the tribe is historically the basic political organization and principal power entity. These traditional institutions evolved through collective use of natural resources, and are based on efficiently implemented rules used to regulate collective ownership and maintain social-ecological resilience (Sheehy et al., 2006).

A migratory system of animal husbandry is structured around a combination of seasonal and ecological variables and environmental heterogeneity of the landscape (Wu, 2000). Herd diversification is a risk-reducing adaptive strategy supported by particular breeding and crossbreeding arrangements (Wu, 2000; Miller, 2002). The proportion of species and the size of herds differ according to grassland factors and the suitability of the landscape for different animals. Grasslands contain various herbaceous species which suit a particular domesticated and wild species, requiring a rotation and separate utilization of ecological zones. In the summer, nomads move to the best pastures on the plateau and mountains and no grazing on winter pastures is allowed. Nomad communities carry out patrols over their entire territory in summer and autumn to investigate violations against customary laws (Norbu, 1997). Traditional grazing practices also have an impact on biodiversity conservation. Much of the grazing ecosystem is intact and sustains a unique flora and wild fauna, despite centuries of grazing, indicating its remarkable resilience (Wu, 2000).

Resilience of the socio-ecological system among nomad societies is further supported by the maintenance of social relationships as the practical base of flexibility and a key to survival 
(Lancaster \& Lancaster, 1998). Nomadic societies are in general presented as passive victims, caught in their traditional way of life and unable to cope with modernization and diversification, ignoring the fact that they are not autarkic, self-sufficient and closed systems (Salzman, 1980, Khazanov, 1998). They depend on a mutual exchange with a non-pastoralist economy and have a local appreciation and acceptance of external innovations and improvements (Khazanov, 1998). The unpredictable environment demands vast networks of information and social relationships based on a moral system that takes primacy over material considerations. Conduct is governed by a respect for cohabitation norms of behaviour handed down from the past that partially stem from ancient religious laws (Norbu, 1997). However, customary natural resource management practices have been greatly transformed by government driven development and conservation projects.

\subsubsection{Socio-cultural change and regional developments}

In many regions throughout the globe the validity and applicability of indigenous practices are not only questioned because of their cultural context, but also denigrated because of the devolution of resource management authority and legitimacy to local communities (Berkes, 2008). The Tibetan people are likely to be the politically most sensitive Chinese minority. Environmental self-determination of Tibetans is a crucial issue as control over natural resources and conservation action is a question of political power (Yeh, 2009). The Chinese government considers the nomadic lifestyle as an obstacle to progress and modernization as nomadic tribes are not easy to control by the central government (Yeh, 2003; Klieger, 2007). Future pathways for conservation and sustainable development cannot ignore human preferences and decisions. Preferences of individuals and communities in land use practices are to a certain extent shaped by economic incentives and interests, but also include cultural interests (Rao et al., 2003). Tibetan cultural identity is closely associated with the grasslands. Many indigenous people on the Tibetan Plateau share a common vulnerability to environmental change, and are caught up in a battle to sustain their cultural identity and autonomy (Kolas \& Thowsen, 2005).

The ideology of development that underlies the official scheme of social and economic policies for the region is based on the assumption that the traditional Tibetan way of life and production is backward or underdeveloped (Yeh, 2009). The government has introduced policies that seek to encourage nomad herders to adopt a sedentary lifestyle by providing them with winter homes, livestock shelters and grassland fencing (Richard et al., 2002). A 
key legal document behind this policy is the Law of Rangelands of the People's Republic of China that defines tenure rights of rangeland used by the herders and outlines the duties and responsibilities to protect, construct, and manage the rangelands. Since the 1980s the government has started with the introduction of a Household Contract Responsibility System in many parts of the region. Land use contracts are granted to individual households as a longterm lease (50 years). These contracts are renewable provided that land management is satisfactory, while ownership of the land remains government property (Richard et al., 2006). However, this law was applied to the livestock sector without consideration for communal land management rights and local variations in the livestock carrying capacity of grassland ecosystems (Foggin, 2000; Yeh, 2003). In addition, livestock is supervised by local administrative departments but unclear property rights and the dominant, paternalistic role of the government is a frequent source of conflict between herders and local authorities (Foggin, 2008). Table 2.1 provides a comparison of policy implementation models for resource tenuremanagement arrangements and their relative strengths and weaknesses (Adapted from Richard et al. (2006):

Table 2.1 Comparison of resource tenure management arrangements

\begin{tabular}{|c|c|c|}
\hline Government Driven & Co-management & Customary Practice \\
\hline $\begin{array}{l}\text { Easier to provide services such } \\
\text { as credit and veterinary care }\end{array}$ & Lower risks/costs per household & $\begin{array}{l}\text { Lack financial resources } \\
\text { and technical inputs }\end{array}$ \\
\hline $\begin{array}{l}\text { Tenure more secure under } \\
\text { situations of conflict and } \\
\text { instability }\end{array}$ & $\begin{array}{l}\text { Legal rights ensured per } \\
\text { household }\end{array}$ & $\begin{array}{l}\text { Individual households lack } \\
\text { equitable rights }\end{array}$ \\
\hline Ignores community strengths & $\begin{array}{l}\text { Subsidies and technical inputs } \\
\text { provided }\end{array}$ & $\begin{array}{l}\text { High but shared labour to } \\
\text { protect traditional pastures }\end{array}$ \\
\hline Creates higher costs/risks & $\begin{array}{l}\text { Decisions regarding management } \\
\text { made by community }\end{array}$ & $\begin{array}{l}\text { Increasing external } \\
\text { encroachment }\end{array}$ \\
\hline $\begin{array}{l}\text { Creates unintended conflicts due } \\
\text { to poor allocation processes }\end{array}$ & $\begin{array}{l}\text { Communities skills are } \\
\text { strengthened (social capital) }\end{array}$ & Greater mobility for grazing \\
\hline $\begin{array}{l}\text { Does not protect large landscape } \\
\text { amenities }\end{array}$ & $\begin{array}{l}\text { More facilitation required, } \\
\text { especially with larger population }\end{array}$ & \\
\hline $\begin{array}{l}\text { Reduced flexibility during dry } \\
\text { years }\end{array}$ & Greater mobility for grazing & \\
\hline
\end{tabular}


The deep transformation or destruction of traditional social, economic, and cultural institutions can have disadvantageous consequences for nomadic people and the larger societies of which they are part (Khazanov, 1998). International development experience has shown recurring failure in both human and environmental terms in programmes that aim to promote sedentary lifestyles in pastoral systems as different case studies about pastoral communities in central Asia show (see e.g. Salzman, 1980; Ginat \& Khazanov (Eds.), 1998; Khazanov \& Wink, (Eds.), 2001). The enclosure of rangelands often leads to severe overstocking and the disappearance of "self-correcting change" based on climate-tracking strategies or risk-minimizing low-stocking approaches (Miller, 2002). Over time, restricting movements and redistribution of communal land holdings due to administrative boundaries have led to increasing human and livestock populations and consequentially to overgrazing on the Tibetan Plateau (Yeh 2003; Richard et al., 2006). In addition, pastoral intensification can also partially be motivated by environmental anxiety (Salzman, 1980). The designation of more land as national parks not only limits the access to traditional resources, but also tries to reduce pastoral activities by downsizing livestock herds, the main economic source of income and a symbol of wealth for nomad families.

However, overgrazing is not often the primary factor of environmental degradation and the resettlement of local herders and restrictions on natural resource use does not usually bring many environmental benefits (Morton, 2008; Tang \& Gavin, 2010). Environmental degradation in general and degradation of grassland conditions have at least partly been caused by natural factors such as decreasing precipitation and a rise of temperatures (Harris, 2008). The adaptation of the traditional lifestyle to changing environmental conditions may contribute to a change of public awareness of environmental changes and a loss in the ability to evaluate long-term trends in the ecosystem (Salzman, 1980; Khazanov, 1998). Further, if the development interests of local people are marginalized, they might adopt actions detrimental to the goal of conservation (Rao et al., 2003). For the Tibetan rangelands there is need for fresh perspectives and information on ecosystem dynamics and pastoral development and which aspects of indigenous knowledge systems can be used in the design of new conservation strategies (Miller, 2002; Richard et al. 2006; Sheehy et al., 2006). At the same time, it is important to improve local livelihoods by development interventions that take into account local pastoral economies (Wu, 2000).

In addition to changes in local land use rights, traditional resource management systems are challenged by the government's economic development plans for the region. The 
measurement of poverty is a highly politicized issue in lower government levels (Gransow, 2009). China has experienced a fundamental change of the national development strategy to decrease regional disparities and reduce poverty in rural areas. However, development processes in the rural areas of the Plateau show insufficient planning and little consideration of the potential impacts (Wu, 2000). As is the case for many developing countries, government and civil society initiatives for environmental conservation in China often run contrary to government initiatives for poverty alleviation, and development and resource exploitation often occurs in the name of development (Ho et al., 2004).

In 2000, the Chinese government launched its "Great Opening of the West Campaign" (xibu da kaifa 西部大开发). The main objective of this campaign is to develop the western part of China and bring living standards closer to those of the coastal region (Lu \& Neilson (Eds.), 2004). The government set out environmental protection ${ }^{7}$ as one of the five main pillars of this campaign (Zheng \& Qian, 2004). The protection of nature and biodiversity is one of five environmental frames, and environmental protection is now a key evaluation criterion for cadre performance (Martens, 2007; Lo \& Tang, 2007). No government-issued documents on the development of China's western provinces are complete without reference to either "environmental stability" or "ecological construction" (Harris, 2008). However, environmental remediation mega-projects under this campaign such as "crops to grassland" or "retire livestock, restore grasslands" focus more on the land conservation aspect and hardly engage with the interests of the local population (Foggin, 2005; Harris, 2008).

The construction of nature reserves under the "Great Opening of the West Campaign" is in most cases associated with huge infrastructural developments to improve regional transport and tourism development (Harris, 2008). Apart from Tibetan culture, the natural environment and the wildlife of the Tibetan Plateau attract Chinese and an increasing number of international tourists. Having a less developed tourism infrastructure than other popular tourist destinations in China, tourism in the Tibetan Plateau has developed at a comparatively slower pace. However, the goal of the Chinese government to increase development of the region under its 'Great Western Development' strategy through tourism becomes clear when considering large-scale infrastructure projects. China already holds the record of the highest located airports in the world and more airports are planned to be built in the region to connect even the remotest places of the Tibetan Plateau to the national transportation and tourism

\footnotetext{
${ }^{7}$ The Great Western Development programme is divided into five mega-projects that focus on the development of infrastructure, environmental protection, sectoral and economic adjustments, human capital, and foreign investment and trade
} 
network. In 2005, the construction of the Qinghai-Tibet railway was completed to connect Qinghai Province's capital Xining to Lhasa. Many of the Chinese tourists and a few international tourists I encountered in Xining were taking the train to Lhasa. Until a few years ago the only ways of transport were by plane, overland by bus or car over long distances on poorly maintained roads. As the railway runs through the main habitat of the chiru, there was much controversy about what impact it might have on the species' migration (Cai, 2008). This shows that the main challenges for protecting the region's biodiversity lie not only in recognizing the problem but also in the way these are prioritized and handled by the government.

\section{$\underline{2.3 \text { Biodiversity conservation in China and the Tibetan Plateau region }}$}

\subsubsection{State institutions for biodiversity conservation}

The ways biodiversity related problems are handled in China are strongly influenced by historical, political, economic, and social challenges (McBeath \& Leng, 2006). Conservation action follows a long period of non-action. Going through a state-led "war against nature" under the Maoist era (Shapiro, 2001), state-led activities to protect the environment began relatively late. It was not until the late 1990s that China experienced a policy shift away from pollution control towards natural resource management (Morton, 2005). A changing attitude towards biodiversity conservation as central criteria for sustainable development is particularly noticeable among Chinese scientists. However, science does not necessarily influence policy (McBeath \& Leng, 2006; Harris, 2008). China's increasing global integration has influence on domestic environmental agendas but there is still a reluctance to accept assistance under stringent environmental issues that run contrary to the country's economic development plans (Mol \&Carter, 2007).

The state apparatus remains of dominant importance in environmental protection and reform. Decisions affecting conservation are made at various administrative levels, with most significant decisions being made at the national and provincial level. The Chinese conservation governance regime is characterized by a 'fragmented authoritarianism', resulting from a lack of integration and centralized unity in the institutional framework (Coggins, 2003). Conservation tasks are often divided among numerous agencies leading to uncoordinated and conflicting policies and procedures in the horizontal and vertical administration of conservation (Morton, 2008). 
The Ministry of Environment (MoE, formerly State Environmental Protection Agency) plays the single most important role in China's environmental politics. Conservation related issues are mostly handled by its department of nature conservation that is the responsible body for nature reserves and species management. The State Forestry Administration (SFA) is responsible for a large number of reserves for the protection of forests or wildlife.

Biodiversity conservation is mainly the responsibility of Department of Fauna and Flora Conservation in the State Forestry Administration. At provincial level, the Environmental Protection Bureau (EPB) is the administrative and supervisory department for ecological and environmental protection while the Forestry Bureau is the administrative department for forestry and wildlife conservation. Wildlife management offices are housed operating under the respective forestry bureaus at provincial level. The MoE has nominal oversight over SFA's management of nature reserves. In view of the fulfilment of international conservation agreements, the Chinese Foreign Ministry has another central function in biodiversity conservation.

China has sought continuous improvements of in its biodiversity conservation regime, away from a rigid command-and-control system of environmental governance through the devolution of administrative power to provinces and autonomous regions (McBeath \& Leng, 2006; Mol \& Carter, 2007). However, there is a considerable variation in the actual on-theground implementation of conservation agendas. There is a particular need to distinguish the differences between the central and local governments, characterized by a strong relationship and interaction between provincial and local level. In case of protected areas, the central government in Beijing provides administrative rules and oversight. One-time funding provided by the government is mainly for the infrastructure, whereas the day-to-day funding responsibility for operation and maintenance of protected areas remains with provincial-, county-, and township-level governments. Financing conservation remains a major difficulty, especially as local governments in the most biodiverse-rich regions are among the poorest in China. The biggest part of practical conservation work is carried out at the prefecture, county, or township level as well. However, authorities at lower government levels are in many cases under-resourced and poorly trained and are often more busy with paperwork than fieldwork (McBeath \& Leng, 2006; Harris, 2008). Biodiversity conservation is further affected by a lack of coordination between protected areas, mismanagement and manipulation by local or regional party officials (Morton, 2008). An additional challenge is the coordination of economic development activities that often run contrary to conservation projects. 
Under Chinese law, biodiversity is considerably well protected. It is on the ground where major problems occur, partly due to a large body of laws and regulations that lacks comprehensiveness (McBeath \& Leng, 2006). The Environmental Protection Law (1979, amended in 1989) is China's basic environmental law. Though, in terms of the protection of biodiversity, the Wildlife Protection Law (1998) is a key legal document. However, the law is focussed on protecting individual animals and gives no attention to habitat conservation outside protected areas. Chinese policy based on the fundamental premises that wildlife should be completely protected from human-caused mortality by a conservation policy based on nature reserves for protection and captive breeding for consumption (Coggin, 2003; Harris, 2008). The wildlife protection law principally alienates people from the wildlife and their environment as it contains many restrictions and has little focus on the rational use of wildlife (Harris, 2008). In addition, it contributes to the erosion of traditions of sustainable use among many non-Han ethnic groups (Harris, 2008).

In terms of biodiversity conservation, China has followed western conservation models by creating large numbers of public protected areas, in which rigid standards are implemented; flagship species identified; and key ecosystems, core areas, buffers, and experimental areas defined (Zhang \& Wang, 2004). Once demarcated, all areas are appropriated by the state (if they are not government-owned already), formally becoming public property (Xu \& Melick, 2007). China has three types of nature reserves: Natural ecosystem, wildlife, and natural monuments. Central criteria for the establishment of nature reserves include diversity of biological species, species' richness, unique ecosystems, and high rates of endemism. Protected areas are proposed by scientists or government agencies, and indigenous people are rarely included in assessment, planning, demarcation, and management decisions (Xu \& Melick, 2007).

\subsubsection{Biodiversity conservation on the Tibetan Plateau}

Proposing and approving protected areas in China's western provinces has become a popular activity characterized by a movement to declare large areas of already settled territory as new nature reserves (Harris, 2008). Government supported conservation in the region began in the 1980s with the establishment of small nature -protected areas. A number of initial smaller sanctuaries have been folded into mega preserves, where they became "core zones" (Fleming et al., 2007). Since then, state-led conservation action mainly took place in 
form of the establishment of "mega-preserves" such as the Qomolangma National Nature Preserve, the Changtang National Nature Preserve, and the Four Great Rivers Ecological Environment Protection Plan (Fleming et al., 2007). Each of the mega preserves has a different conservation focus and implementation plan. According to Fleming et al. (2007), the Tibetan Plateau currently has over 40 percent of its land under conservation management, but its conservation successes are little known outside the region.

The conservation success stories on the Tibetan Plateau were in frequent cases achieved at a great social expense. The establishment of natural protected areas and reserves often has far reaching social and economic consequences, especially in remote rural areas (Martens, 2007). A centralized planning system that is focused on long-term achievements often gives less attention to the everyday demands of on-the-ground conservation. The architecture of protected areas is based on the habit to follow existing patterns rather than break new ground (McBeath \& Leng, 2006). However, a one size fits it all approach is ill suited for China's diverse west. National and international attempts to manage and conserve the various ecosystems found on the Tibetan Plateau have historically come into conflict with the lives and livelihoods of local herders. Conflicts with communities are in most cases related to the regulation of access to land and resources within protected areas, participation in decision making processes and the recognition of local culture, lifestyles and relationships with nature (Harris, 2008).

The establishment of protected areas is a leading example of how the state attempts to control indigenous populations in terms of their property rights and social and ecological interactions (Yeh, 2009). In the past, people and communities within or bordering nature reserves were considered a threat to biodiversity in China and the displacement and resettlement of indigenous people was the general government policy (Harris, 2008). By implementing a three-tiered zoning system ${ }^{8}$, people living in the core zone must be removed by law. People are allowed to live in the experimental and buffer zones on but not off the land which prohibits natural resource use by local people. This contradicts with the nomadic lifestyle of many people and the irony of these conservation efforts is that Tibetan people that have lived on the Plateau for centuries are increasingly viewed as threats to local ecosystems and removed from their ancestral lands (Foggin, 2008).

\footnotetext{
${ }^{8}$ In the three-tired zoning system that the Chinese government applies in its protected areas, a protected area is divided into core, buffer and experimental zones. The different zones allow for different degrees of human interaction with the environment.
} 
The result of these developments is a growing urbanization in mountain areas as many nomads are leaving the grasslands hoping for a better life in the city. These are challenged by the control over demographic growth and a diversification of economic structures for a sustainable performance of suspended villages. Low levels of education and widespread unemployment in the region offer few economic alternatives to nomad herders (Kolas \& Thowsen, 2005). Even though eco-tourism has been widely promoted as an ecological sound source of income, it is poorly managed in many cases. The environment of the Plateau is too harsh and vast to realize ecotourism development based on wildlife viewing and ecotourism in other parts of China rarely translated into sustained economic development for local people (Harris, 2008).

Official publications highlight the government's efforts in encouraging a "harmonious development" (hexie fazhan 和谐发展) for the region that simultaneously provides development benefits and protects the environment (State Council Information Office of the People's Republic of China, 2003; Foreign Culture Exchange Association of Tibet Autonomous Region, 2007). However, conservation governance in China is characterized by a significant gap between national policy rhetoric and its actual implementation on the ground (McBeath \& Leng, 2006; Harris, 2008). It is suggested that a pluralistic approach to environmental management that combines non-state practices with state-led regulations has a stronger potential to combine economic incentives, participatory practices, and state regulation (Morton, 2008). 


\section{$\underline{2.4}$ The role of civil society development for conservation}

\subsubsection{The Chinese NGO community}

McBeath \& Leng (2006) have identified three major linkage mechanisms to bridge the gap between national policy and local implementation. First, by central government coordination, with focus on the role of the State Council, local affairs, reports, law enforcement, development planning; second, by national fiscal policy with regard to government resources and foreign financial assistance and finally, through mobilization campaigns and transmissions channels, such as media and NGOs. The need to better understand the role that non-state actors can play in support of local governance efforts under the specific sociocultural and political context in China is put forward by several scholars (see e.g Morton, 2008; Gransow, 2009). China's economic reform and development provides new opportunities for the execution of environmental improvements by multiple actors. Studies on biodiversity conservation in China have mainly focussed on the national policy level, and the role of the NGO sector for conservation has hardly been addressed. Since the formation of the first environmental NGO (or NGO of any kind) in China, "Friends of Nature", in 1994, Chinese NGOs are becoming increasingly engaged in public debates on environmental issues and civil society participation in conservation (Wu, 2000).

The establishment and active contribution of NGOs in the environmental sector in China, has been hampered by government policies related to organizations. China has strict rules for the establishment of social organisations and the registration process is full of barriers. The government Regulations on the Registration and Administration of Social Organisations stipulate that organisations have to be subject to state control (State Council of the PRC, 1998). The regulations require that each organisation has to register under the aegis of an official state authority, party institution or a public enterprise. These institutions are held responsible for potential slips committed by the organization making the registration process arduous and unsuccessful in many cases. Many organizations decide for this reason to bypass the registration process with the Ministry of Civil Affairs by affiliating directly with research institutes or universities. Others register under false pretences as a private business entity or to remain unregistered and operate "illegally" which is more or less tolerated by the government (Gransow, 2009). This can lead to many problems like a limited access to financial and other resources, as organizations without legal status are prohibited from accepting outside donations and a lack of cooperation with relevant state organs (Zhang \& Baum, 2004). 
The formal affiliation of NGOs with government institutions can have a positive feedback effect on government policies and with direct, unmediated access to international funding sources, NGOs are able to remain financially and operationally independent of the local governments. However, Zhang \& Baum (2004) use a case study of a local NGO in Qinghai to demonstrate that even registered NGOs try to avoid dealing with state agencies directly as local authorities can have difficulties in managing financial resources effectively. The authors argue that the initiation, funding and management of projects are all strongly influenced by donor agencies and NGOs are able to maintain their autonomy and avoid intrusive government interference with donors themselves taking the place as "outside authorities". The donors' external position to the Chinese political system, and their access to major financial resources has enabled a somewhat independent societal space, in which organizations may operate comparatively free from state control given there is no major conflict of interest between the different parties (Zhang \& Baum, 2004).

The NGO community of China is diverse and significant differences exist among NGOs. The NGO community is distinct from other countries in so far that government organized non-governmental organizations (GONGOs) in China play a larger role in civil society development. GONGOs are organizations that are set up by the government to take on NGO features. Although some scholars simply view them as extended organs of the government, GONGOs do not implement projects directly through formal administrative systems but instead they function more as research centres or consultants for government agencies $(\mathrm{Wu}$, 2002). A major reason for the establishment of GONGOs was to receive assistance from multilateral, bilateral or international nongovernmental organizations, however, GONGOs now play a major role in civil society development (Wu, 2002). Compared to NGOs, GONGOs have more freedom of registration and organization due to close links with state agencies and have a major function in bridging the gap between NGOs, civil society, the state and the international community (Mol \& Carter, 2007). Environmental GONGOs such as the China Wildlife Association play a significant role in conservation through their partnerships with international organizations, and are supporting the development of a stronger independent green civil society in China (Wu, 2002). In addition, a positive trend is the development of organizations that were originally initiated by the state to independent organisations (Zhang \& Baum, 2004).

A further characteristic of the Chinese NGO community is that Chinese environmental NGOs seem to be better organized and more influential on both government and civil society 
than organizations operating in other sectors (Lu, 2005; Sun \& Zhao, 2007). Regions in China differ in their environmental performance and compliance. ENGOs can act as a major linking mechanism for central and local conservation governance coordination (McBeath \& Leng, 2006; Morton, 2008). The government promoted concept "small government, big society" concentrates on core government functions and shifts the focus to the increasing responsibilities among the Chinese society (Lo \& Tang, 2007). ENGOs increasingly take over tasks which traditionally were the responsibility of state authorities such as creating public awareness and delivering education about environmental issues (Gransow, 2009). Their major aim is to increase public participation by implementing high-profile education campaigns to influence environmental decision-making that is supported by an increased exchange between NGOs, media, and conservation experts on national and international level (Lu, 2005). However, despite these linkages mechanisms and increasing government endorsement, biodiversity conservation still lacks extensive support from the public (Lo \& Tang, 2007). Biodiversity conservation in China is a government objective. However, it is not fully endorsed by the public and most Chinese people are unwilling to sacrifice economic gains to preserve threatened ecological systems that are not in their backyard (McBeath \& Leng, 2006).

\subsubsection{Potential influence of NGOs on conservation governance}

Supported by the international literature (Ostrom, 2005; Pahl-Wostl, 2009), Lo \& Tang (2007) recommend an analysis of changing institutional and economic contexts to understand environmental management systems and conclude that a wide array of actors is a key ingredient for improving environmental governance. However, the question is how these theories apply to China where the state has ultimate political authority and non-state approaches to environmental management still require strong political commitment from the state. There is a controversy among scholars regarding what real influence NGOs can have on government policies in China (Morton, 2005; McBeath \& Leng, 2006). Public participation is a complex and politically sensitive task as it relies upon broader developments within Chinese civil society and remains heavily influenced by the power relations that are typically associated with an authoritarian regime (Martens, 2007). NGO's freedom of operation remains particularly limited in relation to minority and human rights issues (Oberheitmann, 2009). In view of these limitations, NGOs generally base their function within Chinese civil society development on a moderate ‘self-constrained advocacy' approach (McBeth \& Leng, 
2006; Sun, Zhao, 2007). Most adhere to the Confucian principle of respect for authority and status, do not aim to challenge the government and point out the importance of collaborating with state authorities and helping it with finding solutions to environmental problems ( $\mathrm{Lu}$, 2005). There has no doubt been an expanded activism of NGOs in the field of environmental conservation over the last 15 years; however, scholars argue that this is partly because of an increase in environmental problems (Lu, 2005). Environmental activists are more consciously seeking to influence government policy and decision-making, leading to a subtle transformation of China's governance institutions and a reconciliation of tension between local autonomy and vertical accountability (Lo \& Tang, 2007).

Managing environmental problems requires a long-term commitment towards building political consensus, institutions, and public awareness (Morton, 2005). 'Guangxi, understood as networks of informal social norms, rules and unwritten codes of conduct, play important role for organizing social life in China (Mol \& Carter, 2007). The type of interaction between NGOs and the government institutions responsible for them can differ greatly, with sometimes close personnel links (Gransow, 2009). The formation of informal rules and social networks around conservation programmes can also differ among different geographic regions. A major question is how, or if, a diverse range of relationships can actually strengthen capacity to pursue environmental goals more effectively. It is argued that civil society development in China is characterized by an absence of a coherent environmental movement, partly owing to a lack of ability to coordinate among different organizations, in particular a lack of cooperation with NGOs in other areas such as poverty alleviation, and pooling resources (Wu, 2002; McBeth \& Leng, 2006). This is further challenged by a competition for financial resources and a politicization of environmental issues ( $\mathrm{Lu}, 2005)$. The compliance and capacity at local level is of critical importance for success of conservation projects. In some cases village governments serve as autonomous social and political organizations to replace a poorly-develop NGO network in China (Coggins, 2003). NGOs are very diverse in size and the scale they operate, consequentially their ability to act as bridging organizations will also vary.

Environmental policy making at the national level is increasingly influenced by international and supra-national institutions and a number of NGOs at the national level are affiliated with international organizations. More recently, international assistance in the environmental sphere has shifted towards being more grassroots oriented (Morton, 2005). A reason for this shift is related to concerns about the lack of transparency of Chinese 
government partners and negative experiences with big government-led projects in other parts of the developing world (Wu, 2002). Consequently, in order to obtain more international grants and technical assistance for environmental projects, the Chinese government often has to guarantee the participation of NGOs in the project's implementation (Wu, 2002, p.50).

Direct foreign influence and involvement through international NGOs in conservation has a long history on the Tibetan Plateau. For instance, the protection of the chiru (Tibetan Antelope) received much attention in the late 1990s from both the international community and Chinese authorities after chiru populations faced a decline through poaching and illegal wildlife trade (Harris, 2008). International NGOs helped to raise awareness to the plight of the chiru and other endangered species of the Tibetan Plateau internationally, while it was the responsibility of local grassroots organizations to implement action on the ground.

In addition to the participation of grassroots NGOs, international organisations often call for the involvement of local people in conservation but China offers no structural channels for citizens to become involved in environmental policy and management (Martens, 2007; Morton, 2008). Case studies in other regions of China have described the changing socioeconomic and political context for conservation as beneficial for the introduction of participatory methods (Vernooy et al., 2006; Xu et al., 2006). The employment of local people in conservation management is considered to be a cost-effective way to harmonize economic land use practices and nature conservation (Coggins, 2003). This form of local involvement, while not directly linked to conservation governance, requires the recognition of local cultural traditions, environmental knowledge, and land use practices, and also enforces an understanding between resource utilization and biodiversity. There is a common agreement among Chinese and foreign scientists that traditional pastoral responses to ecosystem demands may be the most appropriate responses and that pastoralism can generally be pursued without great loss of biodiversity by promoting a sustainable coexistence through regulated and monitored use (Foggin \& Smith, 2000; Sheehy et al., 2006; Harris, 2008; Berger, 2010).

It was not until recently that NGOs and government agencies realized that communities can play a positive role in conservation. In more recent times, co-management had become a commonplace term in discussions of Chinese nature reserve management followed by some experimentation with community co-management of nature reserves. The Guidelines of Community-based Natural Resources Co-management in Nature Reserves prepared by the Department of Wildlife Conservation of the State Forestry Administration, defines co- 
management as "the process for sharing decision-making, implementation and evaluation of nature reserve management programs" (State Forestry Agency Department of Wildlife Conservation, 2002). However, Chinese authorities and NGOs have their own interpretation of "co-management", and local residents are in a number of projects more seen as target groups, not partners (Martens, 2007). In addition, while western scholars highlight the powersharing aspect of co-management, some Chinese scholars (e.g. Zhang \& Wang, 2004) regard the main reason for a co-management approach to provide local communities with economic alternatives to help them reduce their dependence on natural resources. Nevertheless, improvements have been made in environmental education and training local communities in conservation issues. For example, the All-Tibet Conservation Training Center in Lhasa was established to train people in various conservation tasks (Fleming et al., 2007). In addition to environmental training, communication and coordination are of very important and improvements have been made for communication infrastructures and for gathering and storing data in remote regions on the Tibetan Plateau (Fleming et al., 2007).

\section{$\underline{2.5 \text { Summary }}$}

The Tibetan Plateau provides a mixed picture of the current ecological crisis and a diversity of challenges related to biodiversity conservation and its governance. First of all, the importance of the conservation of the region's biodiversity has been acknowledged by both government and civil society. However, the administration and enforcement of conservation agendas on side of the government is challenged by a complex institutional environment and legal deficiencies. Financial and human resources needed for carrying out conservation work at lower governance levels is highly dependent on outside assistance. The common architecture of protected areas has proved to be ill suited for the region's local socio-cultural and economic requirements based on nomadic pastoralism. On the community level, traditional management practices further struggle to deal with global phenomenon such as climate change and the government's conservation and development plans for the region. Civil society is an emerging concept in China and has increasing influence on conservation issues and stakeholder communication but still underlies significant limitations in its development. Even if the various challenges that have been identified in this chapter have considerable impact on conservation, the focus of my research will be on those that belong to the conservation governance spectrum . 


\section{Chapter III: Methodology}

\section{$\underline{\text { 3.1 Introduction }}$}

The previous chapters have described the framework for biodiversity conservation on the Tibetan Plateau based on theoretical approaches towards conservation governance and empirical studies on conservation related issues in the region. An interdisciplinary approach that combines social sciences, humanities and cultural studies is indispensable for this research in view of the various ecological, social and political factors that have influence on biodiversity conservation in the region. Multiple qualitative methods were applied to triangulate and cross-check research findings. I will first describe the selection process for the Tibetan Plateau as a case study, and then I will explain how qualitative methods in form of a literature review, semi-structured interviews and information material from NGOs were used in this research. I will then elaborate on ethical considerations associated with the research and how these were addressed. In the last part of this chapter I will describe how I analyzed the data collected.

\section{$\underline{3.2}$ Research design}

\subsubsection{Site selection for case study}

The Tibetan Plateau region in China, encompassing the Tibet Autonomous Region, most parts of Qinghai and western Sichuan Province as well as small parts of Yunnan and Gansu Province, was selected for a case study approach. Although parts of the Tibetan Plateau are in Bhutan, India, Nepal and Pakistan, the majority of the Tibetan Plateau lies within the boundaries of the People's Republic of China. Therefore, I will exclusively focus on conservation arrangements within the Chinese political realm. Having an academic background in modern Chinese studies, it seemed to me reasonable to select a research environment that I am already familiar with and to build on a previous research experience in environmental conservation issues in China's west and other parts of the country. The familiarity with the country's language not only allows me to engage with key literature and other information that is only accessible in the Chinese language but also to communicate with local people as Mandarin is widely understood on the Tibetan Plateau.

The Tibetan Plateau region has been chosen as research location because it appears to display examples of successful case studies on collaboration on biodiversity conservation and 
illustrates many of the theoretical concerns of adaptive governance, co-management, and resilience in social-ecological systems. The region is further of particular interest for this research in view of current changes in socio-ecological relations and development patterns as outlined in the background chapter. The social response to these changes is not only a selforganization for conservation at the local level but it also involves interaction between organizations and institutions at national, and international levels with an increasing expansion of NGO activities. The research focuses more on the institutional dynamics behind biodiversity conservation on the Tibetan Plateau rather than on the local realities on the ground. I assumed that looking at biodiversity conservation governance at a regional scale further increases the objectivity of the research.

The following two maps show the topography of the Tibetan Plateau (Figure 3.1) and its political division (Figure 3.2). A comparison of the maps shows that the Plateau's geographical size nearly overlaps with Tibetan autonomous divisions of governance.

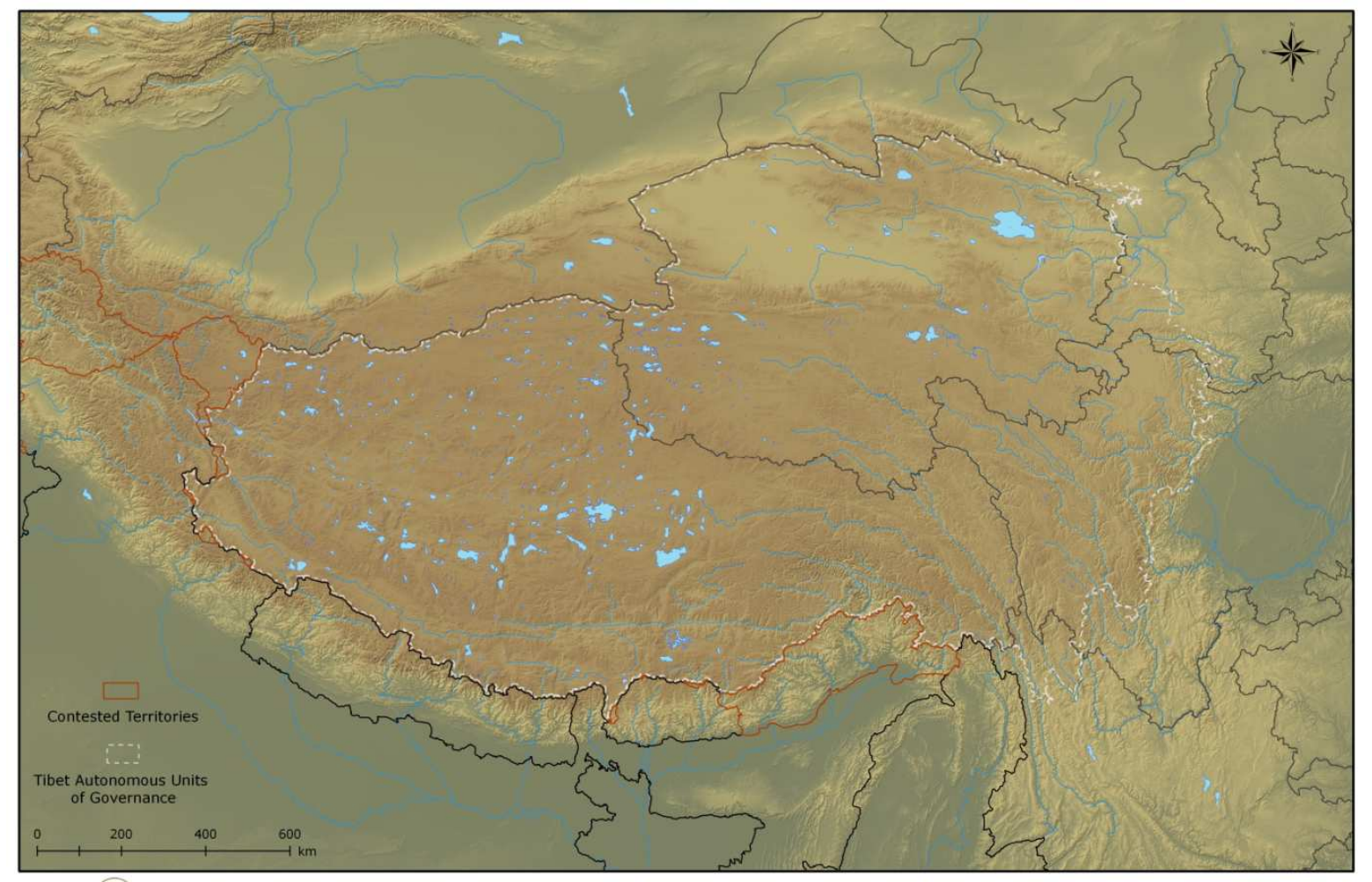

2009 (c) Tibetan \& Himalayan Library (http://www.thlib.org * THL-ID: M43)

Figure 3. 1: Tibetan Plateau and adjoining areas terrain map (Adapted from Tibetan \& Himalayan Library). 


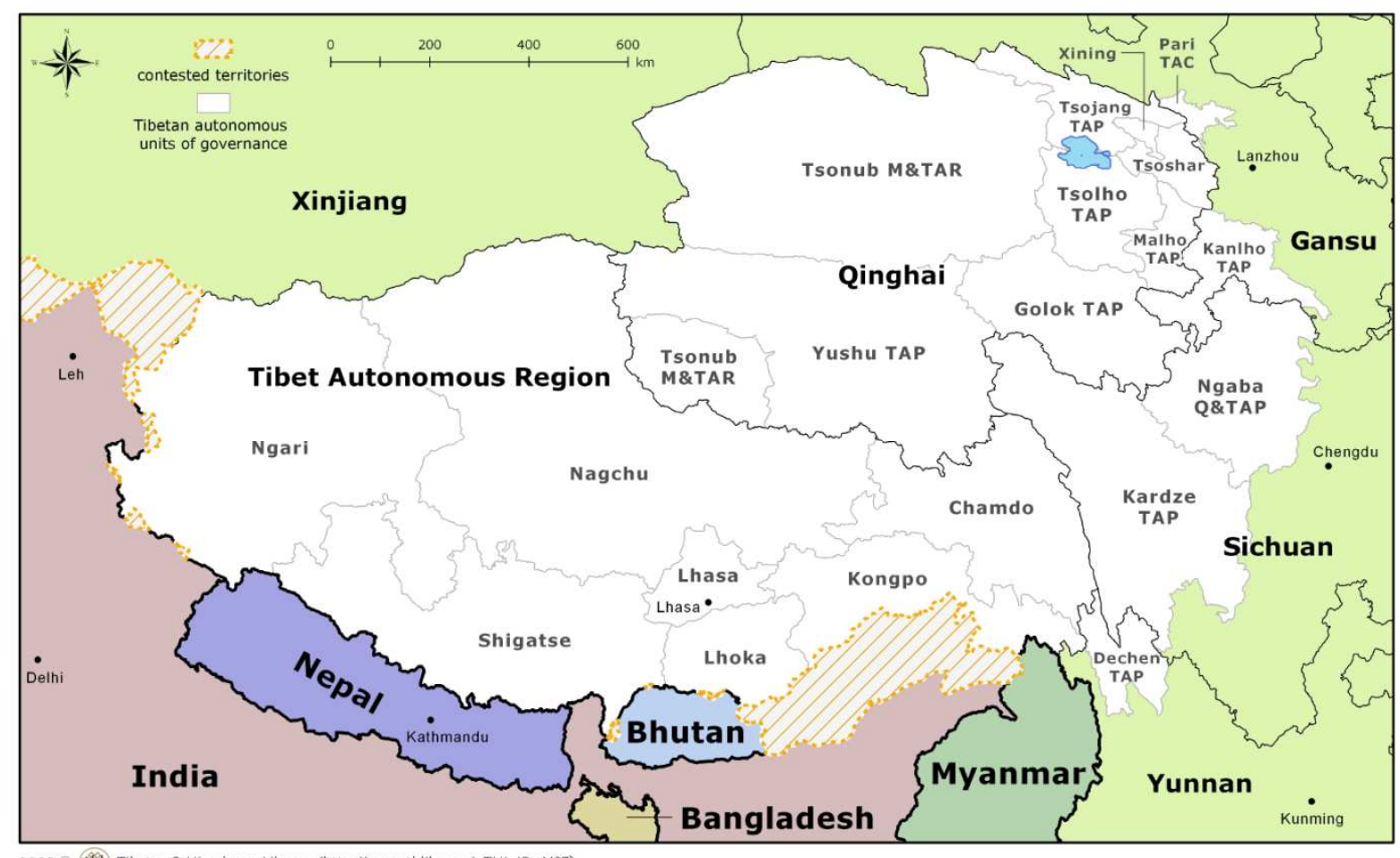

2009 (c) Thetan \& Himalayan Library (http://www, thliborg * THL-ID: M37)

Figure 3.2: Tibetan autonomous units of governance (Adapted from Tibetan \& Himalayan Library).

\subsubsection{Qualitative methods}

\section{Review of academic literature}

A literature review was used to build the conceptual framework for my research and to provide background information on the various ecological, socio-cultural, and political variables for biodiversity conservation in the research location. I used the Tibetan and Himalayan Library as a research tool for digital publications on the Tibetan Plateau region. In addition, the homepage of the Tibet Research Centre of Case Western Reserve University and Tibetanplateau.org provided a list with recent publications from different disciplines on regional development and conservation issues. I also used Google Scholar for searching scholarly literature on the relevant issues for this research. The research study sets out to contribute to an interdisciplinary body of knowledge on biodiversity conservation. As outlined in the introduction chapter, the main theories behind my research are grounded in the shared conceptual framework of biodiversity conservation governance, socio-ecological resilience, and adaptive co-management with particular focus on the role of NGOs as bridging organizations. My research builds on previous studies undertaken in the field of biodiversity 
conservation governance in China in general and of case studies on natural resource management from the Tibetan Plateau region in particular. To understand historical and more recent developments in conservation governance within China and within the Tibetan Plateau context, the literature review was used to analyse informal and formal institutional arrangements for conservation and their dynamic changes over the last years - with a particular emphasis on the role of NGOs in the conservation process. The literature review was used to answer question related to the development of conservation governance in China and the Tibetan Plateau region. It further answered questions related to civil society development in China and the potential role of NGOs in biodiversity conservation. In addition, the literature review provided insights from case studies on bridging organizations in other parts in the world (see Brown, 1991; Hahn et al., 2006, Jamal et al., 2007).

\section{Semi-structured interviews}

Field research in China was undertaken from July to September 2010 to collect data in the form of semi-structured interviews. Bernard (2002) recommends that in situations where it is likely that one will not get more than one chance to interview someone, semi-structured interviews are the most suitable approach. Even though based on clear instructions in form of an interview guide, semi-structured interview are open to interview participants input on other issues and allows following new leads (Bernard, 2002).

Compared to other regions in China, only a few NGOs are active in conservation in the region. Both national and international NGOs working in the field of biodiversity conservation in the Tibetan plateau were selected as the main informants for the research. First of all, it was assumed that NGOs are more approachable than government authorities and local communities. Second, owing to the diversity of local communities and lifestyles on the Tibetan Plateau, it is difficult to make comparative statements by selecting a communitybased approach. The NGOs that are represented in this research have work experience with a wide array of communities in different locations on the Plateau. By operating within the intermediate level between government, donor and civil society, they further provide information about the interaction between the main stakeholder groups in conservation. As outlined in the previous chapters, there exists a research gap in the understanding of the role of NGOs in conservation governance in China and in the Tibetan Plateau region in particular. In addition, there exists a gap in terms of understanding what the key triggers and barriers to bridging institutions are. These gaps will be addressed by investigating single case studies of 
conservation projects for similarities and differences in experiences of NGOs in their function as bridging organizations.

I carried out semi-structured interviews with representatives from eight non-governmental organizations that have project activities in the Tibetan Plateau region and a university-based research that could provide me with information about civil society development in China. Contact with relevant interview partners was established in advance by telephone or email or by using the snowball sampling technique (Silverman, 2010) where other research participants referred me to a person of an organization of interest. This technique was chosen as personal relationships are of high importance in China and facilitate access to key informants.

All but one of the interviews were carried out face-to-face. Interview participants were asked to provide me with information about their organization's environmental conservation or development related work in the Tibetan Plateau region or in China in general. First of all, I asked interviewees about the context specific conservation governance arrangements in their project location(s). Then, I continued with investigating the various degrees of participation of stakeholder groups in the conservation process and how authority and responsibility are shared between different stakeholders. After that, I asked research participants about the role of traditional resource management institutions in their projects and what they perceive as the main triggers and barriers to their inclusion in regional conservation approaches. My concluding questions looked at the NGO's understanding of their bridging function in regional conservation governance and their support of traditional resource management institutions. Interview partners from the China Association for NGO Cooperation and the Tsinghua University NGO Research Centre were asked more general questions related to civil society development and the role of NGOs in conservation in China, with particular focus on NGO and government cooperation on conservation issues.

Interviews were undertaken in Beijing, Qinghai and Sichuan province and lasted between 1 and 2 hours. Interview participants were in all but three cases Chinese nationals. All interviews were carried out in English after non-native speakers assured that they feel comfortable with the language. Interviews were carried out with one person from each organization in all but one case, where I interviewed two representatives. To keep the identity of all informants confidential, I will refer to research participants in the following as an official representative of the organisation. All interviews were based on an interview schedule (see Appendix 2) to lead the conversation to subjects of interest while at the same time 
leaving enough space for discussion and openness to other issues of relevance for the research (Silverman, 2010).

\section{Selection of NGOs to interview}

The priority of this research is not a generalization of research findings or providing a categorical truth based on quantity of data but rather in looking at the single cases in detail to capture a diverse range of viewpoints based on the experiences of NGOs (Silverman, 2010). To narrow the number of informants, NGOs were selected according to conservation-related project activities and years of working experience in the Tibetan Plateau region. The Bridge Fund, Friend of Rural Community Development and Plateau Perspectives are exclusively working in the Tibetan Plateau region; Future Generations and Shan Shui also work in other parts of China but mainly focus on the Tibetan Plateau region, the China Association for NGO Cooperation, the China Exploration and Research Society and Flora and Fauna International have a focus on conservation and development issues in China's Western provinces. Organizations were also selected according to their project activities i.e. their work on co-management and traditional resource management institutions.

The research focuses on issues related to biodiversity conservation; however, in view of the strong socio-ecological relationships on the Plateau it is indispensable to develop a more holistic picture of recent developments in the region. To capture a diverse range of informants, I interviewed people from environmental NGOs (ENGOs), development NGOs and from a government organized NGO (GONGO). Organizations were of local, national or international nature and officially registered with the Chinese authorities.

In addition, my sample size was limited by availability of NGO staff. The months available for my field research overlapped with the field season of many NGOs, and in some cases a large number of staff was not available for interviews. This was mainly the case of representatives from local NGOs that often have a very limited staff capacity. In addition, many NGOs at the grassroots level are not registered (McBeth \& Leng, 2006), and therefore were not included in the study. Consequently, local NGOs are less represented in this research than national or international NGOs. In addition, my selection of NGOs to interview was limited by the time available for interviews and the need to travel large distances to reach many of the organisations active in the field. 
Table 3.1 provides a list of the organizations I interviewed and some additional information about their background:

Table 3.1: List of interview participants

\begin{tabular}{|c|c|c|c|c|}
\hline Name of organization & $\begin{array}{c}\text { Nature of } \\
\text { organization }\end{array}$ & Area focus & Project focus & Project activities \\
\hline Bridge Fund & $\begin{array}{l}\text { International } \\
\text { development } \\
\text { NGO }\end{array}$ & $\begin{array}{l}\text { Works } \\
\text { exclusively in } \\
\text { Tibetan Plateau } \\
\text { region }\end{array}$ & $\begin{array}{l}\text { Rural development, } \\
\text { cultural and } \\
\text { environmental } \\
\text { conservation }\end{array}$ & $\begin{array}{l}\text { Community- } \\
\text { based } \\
\text { conservation }\end{array}$ \\
\hline $\begin{array}{l}\text { China Association for } \\
\text { NGO Cooperation } \\
\text { (CANGO) }\end{array}$ & $\begin{array}{l}\text { National } \\
\text { network for } \\
\text { NGOs } \\
\text { (GONGO) }\end{array}$ & $\begin{array}{l}\text { China's Western } \\
\text { Provinces }\end{array}$ & $\begin{array}{l}\text { NGO networking and } \\
\text { government } \\
\text { cooperation }\end{array}$ & $\begin{array}{l}\text { Participatory } \\
\text { development, } \\
\text { capacity building }\end{array}$ \\
\hline $\begin{array}{l}\text { China Exploration and } \\
\text { Research Society } \\
(\mathrm{CERS})\end{array}$ & $\begin{array}{l}\text { National } \\
\text { development } \\
\text { NGO (Hong } \\
\text { Kong) }\end{array}$ & $\begin{array}{l}\text { China's Western } \\
\text { Provinces }\end{array}$ & $\begin{array}{l}\text { Environmental and } \\
\text { cultural conservation }\end{array}$ & $\begin{array}{l}\text { Research } \\
\text { approach, } \\
\text { Community } \\
\text { participation }\end{array}$ \\
\hline $\begin{array}{l}\text { Fauna and Flora } \\
\text { International (FFI) }\end{array}$ & $\begin{array}{l}\text { International } \\
\text { conservation } \\
\text { NGO }\end{array}$ & $\begin{array}{l}\text { China's Western } \\
\text { Provinces }\end{array}$ & $\begin{array}{l}\text { Biodiversity } \\
\text { conservation }\end{array}$ & Co-management \\
\hline $\begin{array}{l}\text { Friend of Rural } \\
\text { Community } \\
\text { Development (FRCD) }\end{array}$ & $\begin{array}{l}\text { Local } \\
\text { development } \\
\text { NGO (Qinghai) }\end{array}$ & $\begin{array}{l}\text { Prefectures in } \\
\text { north-east } \\
\text { Qinghai Province }\end{array}$ & Rural development & $\begin{array}{l}\text { Community- } \\
\text { based } \\
\text { conservation in } \\
\text { planning }\end{array}$ \\
\hline $\begin{array}{l}\text { Future Generations } \\
\text { China }\end{array}$ & $\begin{array}{l}\text { International } \\
\text { environmental } \\
\text { NGO }\end{array}$ & $\begin{array}{l}\text { Project focus on } \\
\text { Tibetan Plateau } \\
\text { region }\end{array}$ & $\begin{array}{l}\text { Biodiversity } \\
\text { conservation and } \\
\text { rural development }\end{array}$ & $\begin{array}{l}\text { Community- } \\
\text { based } \\
\text { conservation }\end{array}$ \\
\hline $\begin{array}{l}\text { Tsinghua University } \\
\text { NGO Research Center } \\
\text { (NGORC) }\end{array}$ & $\begin{array}{l}\text { National } \\
\text { Research Center }\end{array}$ & National & $\begin{array}{l}\text { Research on NGO } \\
\text { development in China }\end{array}$ & \\
\hline Plateau Perspectives & $\begin{array}{l}\text { International } \\
\text { development } \\
\text { NGO }\end{array}$ & $\begin{array}{l}\text { Works } \\
\text { exclusively in } \\
\text { Yushu TAP }\end{array}$ & $\begin{array}{l}\text { Biodiversity } \\
\text { conservation and } \\
\text { rural development }\end{array}$ & Co-management \\
\hline $\begin{array}{l}\text { Shan Shui Conservation } \\
\text { Center for Nature \& } \\
\text { Society }\end{array}$ & $\begin{array}{l}\text { National } \\
\text { conservation } \\
\text { NGO }\end{array}$ & $\begin{array}{l}\text { Project focus on } \\
\text { Tibetan Plateau } \\
\text { region }\end{array}$ & $\begin{array}{l}\text { Biodiversity } \\
\text { conservation, } \\
\text { Traditional } \\
\text { institutions }\end{array}$ & $\begin{array}{l}\text { Community- } \\
\text { based } \\
\text { conservation }\end{array}$ \\
\hline
\end{tabular}




\section{Official publications}

To supplement the information NGOs provided to me in the form of semi-structured interviews, I analyzed printed and electronic material from the NGOs such as annual reports, information maps, newsletters and the organizations' webpages. Besides the project activities of the NGOs that I have interviewed, there exists a variety of conservation or development projects in the Tibetan Plateau region. I used Google as an internet research tool to investigate information related to biodiversity conservation projects implemented by other organizations.

Methods for data collection also included an analysis of official policy documents of the Chinese government. Official publications and reports were used to investigate government viewpoints and agendas on conservation and development issues on the Tibetan Plateau. This analysis was further supported with an examination of publications from Chinese government controlled media such as the Xinhua news agency, China's Tibet editorial or the Foreign Culture Exchange Association of Tibet Autonomous Region. The China and foreign webbased media network such as tibetanplateau.org, the third pole blog of chinadialogue.net, chinadevelopmentbrief.com and the China Programme of asienhaus.de were used to follow up discussions and publications on conservation issues on the Tibetan Plateau and civil society development in China. I looked in particular at what key threats for conservation the foreign media identifies for the Tibetan Plateau and in how far these are identical to the official viewpoint of the government.

Resulting from the internet- based research and from my qualitative research I developed a profile of the different actors involved in biodiversity conservation on the Tibetan Plateau. The main purpose of the profile is to provide a general overview of the different biodiversity conservation projects currently in place or that have recently been completed, and to identify key stakeholder groups in the governmental and non-governmental environment. Other projects further provided information about conservation-related challenges and on cooperation among key stakeholder groups.

\section{Field diary}

As Silverman (2010) notes, personal observation is also a critical tool in the research process. I spent an essential part of my fieldwork visiting rural areas and conservation relevant sites of the Tibetan Plateau region (particularly in the regions of Amdo and Kham) to 
develop an understanding of the natural and socio-cultural environment of the Plateau. Bernard (2002) describes field notes as the difference between fieldwork and field experience. My field diary was mainly used to note down my personal experiences from engaging with local people and develop an understanding of the different lifestyles on the Plateau.

\subsection{Ethical considerations}

My research was approved by the Human Ethics Committee of Victoria University of Wellington (Approval number: 17833; see Appendix 3). Research undertaken in a foreign cultural environment requires a huge degree of cultural sensitivity and intercultural competence. China has a huge variety of different cultures with different points of view related to their socio-cultural and ecological environment. Being a sinologist by background, I have developed my own viewpoint towards Chinese society and politics. To develop a more objective research approach, it was necessary to take a step back from what I have already learned, and to be open to different realities and new concepts. For this reason, the major part of the conceptual framework of my research was established after the conclusion of the field research.

It is obvious from my physical appearance that I am a foreigner. Consequently, I have to be aware that culturally determined thinking patterns and differences in ontology and epistemology might influence research design and outcomes (Yeh, 2006). A further consideration is the dichotomy between western science and indigenous knowledge for biodiversity conservation. The lack of its cultural context and a further acceptance of "indigenous science" requires respecting and adhering to local language, customs and values (Shackeroff \& Campell, 2007). To win participants' confidence, it was helpful to explain my background and my understanding of the socio-cultural surroundings of the research topic and why I consider it to be important.

Research was not only undertaken in different cultures, but also in a different political environment. The sensitive political environment required caution and considerate interaction with the research participants especially in view of the transmission and publication of local opinions and knowledge (Yeh, 2006). First of all, conservation governance touches upon critical issues such as land use and local political rights. Second, my research location can be classified as a politically sensitive region, which is particularly the case for parts belonging to the Tibet Autonomous Region. To protect my interview informants from any possible harm, I 
did not touch upon issues which could make the informant feel uncomfortable or put the person at any risk. I restrained from asking questions that related to human rights issues and asked interviewees to represent the position of the organization, not their personal opinion.

Research participants were fully informed about the nature of the research in advance of the interview. Participants were given an information sheet (see Appendix 4) and asked to sign an informed consent form (see Appendix 5) to meet the Human Ethics requirements of Victoria University of Wellington. In addition, reciprocity is an important concept in Chinese society. The benefit I will get from and what I will give back to the people that participated in the research has to be taken into consideration. All research participants will be provided with a summary of the major research findings.

\subsection{Data analysis}

My main unit of analysis was conservation area programs, defined as an institution of decision-making and implementation of biodiversity-related activities. The focus is herein on conservation intervention in rural areas encompassing non-urban ecosystems, agricultural villages, pastoral communities, and the system of conservation areas established at the national, local, and non-governmental level. A latent content analysis was used to search documents for themes and determination of underlying meanings. Data drawn from the literature research and review of NGO and government documents were used to triangulate the analysis of semi-structured interviews. Following a comprehensive data treatment, anomalies or deviant cases were actively sought out and addressed.

\subsubsection{Pattern coding}

Data derived from the interviews was analyzed qualitatively based on the procedures outlined by Strauss and Corbin (2008) for pattern coding. Pattern coding was used as tool for identifying the main elements in the data, examining relationships between the different elements and arranging them into a theoretical scheme. A case-oriented constant comparative approach was used to compare findings within one category to the findings within the same category of other interviews to identify similarities and differences in common themes and test out a provisional hypothesis (Silverman, 2010). Through the use of an interview schedule, the interviews were structured according to different descriptive categories and subcategories. First, I analyzed how the predefined subjects of interest were addressed. Then I examined new 
concepts and hypotheses that arose during the interview which were not on the schedule but of potential relevance to the research.

The analytic categories based on the interview sections are listed as follows:

1. Conservation and/or development objectives and related activities

2. Criteria for the selection of conservation project approaches

3. Cooperation with government authorities

4. Role of communities and traditional resource management institutions

5. Funding arrangements

6. Major project challenges

7. Communication between different stakeholders

8. Self-perception of NGOs in the conservation process

\subsubsection{Micro-analysis of social networks}

A major aim of the interviews was to investigate how networks function across the diversity of multiple scales and values in the ecological and socio-economic landscape of the Tibetan Plateau region. Social network analysis can also be used in guiding governing agencies' communication and engagement efforts to maximize efficiency and to target specific subgroups (Mertens et al., 2005). My research applies an "egocentric" network approach which concentrates on the ties surrounding and including one actor in the network, in this case NGOs, opposed to a whole network approach that investigates the complete set of ties among all actors in the network (Kilduff \& Tsai, 2003). In this study, the different governance levels are defined as local, national, and international. Stakeholders at the local level are primarily the local population, local authorities and grassroots organizations. I am applying 'local community' as a general term for the local population, despite the fact that vast parts of the rural areas on the Tibetan Plateau are characterized by nomadic tribes and the term 'community' can be misleading in this context (Rossabi, 2004; Kolas \& Towsen, 2005; Klieger, 2007). The national level includes government bodies and their regional representatives, national research institutes, the national donor society and Chinese NGOs. The international level refers to international NGOs, research associates and the international donor society. It is within this vertical setting and through a horizontal analysis, that I explore the role that NGOs are playing in the process of institutional change for biodiversity conservation on the Tibetan Plateau in favour of local community institutions. While 
investigating NGOs influence on conservation co-management, I make a distinction between collaborative arrangements that involve multiple stakeholder groups and collaborative arrangements that are focused exclusively on local community participation in conservation.

The 'reachability' of a network is the relative ease with which actors can reach other actors in the network (Kilduff \& Tsai, 2003). The first approach for analysing the regional conservation network investigates the variety of ways in which NGOs are linked vertically and horizontally with other organizations at local, national, and international level. For instance, this set includes working with local people, cooperation with government authorities, partnering with nationally and internationally recognized scientists, as well as cooperating with other NGOs. The second approach represents the institutional environment in which local institutions are linked to local, national, and international arenas through working with NGOs. I chose this approach supported by the literature showing that it is mainly through working with NGOs that local biodiversity conservation projects around the work link themselves to local, national, and international arenas (Berkes, 2009; Bodin \& Crona, 2009; Pahl-Wostl, 2009). 


\section{Chapter IV: Results}

\section{$\underline{4.1 \text { Introduction }}$}

This chapter sets out the results of my research based on the qualitative methodologies that were outlined in chapter III. The first part of the chapter outlines the different forms of conservation approaches that are currently in place in the region. I will analyse how conservation governance arrangements are negotiated among different stakeholder groups and identify the means of cooperation that exist among these groups. The second part focuses on the interaction between the different stakeholder groups. The network analysis explores how communication of conservation objectives functions across the diversity of multiple institutional scales, stakeholders and values. As part of the analysis, I will look in particular at the role that NGOs are playing in facilitating communication between different stakeholders. In this final section I will also outline the triggers and barriers to more collaborative conservation approaches associated with the diverse network of conservation stakeholders.

\subsection{Conservation governance on the Tibetan Plateau}

Conservation management must confront various sources of complexity in systems, including their ecological, economic, social, political and organizational components and interactions. Adaptive governance is a combination of disciplinary approaches based on multiple ways of knowing and understanding for solving problems by top-down control of decision making and incomplete knowledge (Gunderson et al. (Eds.), 2010). As discussed in the previous chapters, biodiversity conservation in the Tibetan Plateau region faces a diverse range of challenges and the identification and implementation of adequate conservation governance arrangements for biodiversity conservation on the Tibetan Plateau is a challenge in itself. In addition, based on the experience of the NGOs I surveyed, finding the right balance between preservation and progress, between the needs of human beings and wildlife populations and between modernization and tradition are key criteria for successful biodiversity conservation and sustainable development on the Plateau. In this part of the results, I will start with the investigation of NGOs' perspectives on mainstream government conservation approaches in the Tibetan Plateau region. Then, I will outline the different mechanisms that NGOs use for negotiating conservation governance arrangements towards more cooperative conservation approaches. This includes details on what methods NGOs use to empower communities and to represent their interests, how NGOs approach traditional 
resource management institutions in the conservation process and their efforts in reconciling conservation and development interests.

\subsubsection{Government approaches towards conservation and NGOs' responses}

The Tibetan Plateau receives much attention from the national government in terms of the need for environmental conservation, especially after being promoted the 'Water tower of China' where three of China's major rivers, the Huanghe (Yellow River), Changjiang (Yangtze) and Lancang Jiang (Mekong) have their origin (Harris, 2008). As the need for immediate conservation action on the Tibetan Plateau has been acknowledged by both government and civil society, the question is what needs to be done to protect the region's biodiversity. There is a widespread understanding that environmental conservation strategies for the Tibetan Plateau need to encompass a broad scale as well as implement programs at a level at which natural systems operate (Fleming et al., 2007). However, there is no common agreement on who should bear what conservation responsibilities. Based on the literature review and the responses from my interview partners, biodiversity conservation on the Tibetan Plateau takes place in the following variations:

1. Management by nature reserve only

2. Joint management by government and community

3. Community-based management, overseen by government

4. Management by local traditional institutions

In the first situation, the government has full authority over conservation related activities within the protected area. The second situation describes a collaborative conservation approach where conservation tasks are shared between government and communities. In the third approach, conservation is fully managed by communities and conservation tasks are usually outlined in a contract between government and community. In the last situation, local people manage their resources based on traditional institutions and the government has little or no influence on decision-making processes among local resource users.

A major question is how to balance the different scales of legitimacy and authority in regional conservation governance. Qualitative analysis of the characteristic conservation approaches currently in place in the region reveals that a discourse of administrative and financial rationality prevails across the ecological and socio-economic landscape of the region. Government biodiversity conservation policies that focus on the establishment of protected 
areas, which have mushroomed all over the region during the last decade, leave little space for the decentralization and democratization of biodiversity conservation governance (Harris, 2008).

An example of the simplistic government conservation approaches based on the protected area model is the Sanjiangyuan National Nature Reserve (SNNR) (Sanjiangyuan guojiaji ziranbaohuqu 三江源国家级自然保护区) in Qinghai Province. The Sanjiangyuan region of Qinghai province has a strategically important role in China's Great Opening of the West Campaign. According to the programme plan, development in China's Western provinces should not only create a quality natural environment for sustainable economic development, but also improve the eco-security of south-east Asia and China. Translated literally, Sanjiangyuan means "origin of the three rivers". It is home of the headwaters of the Huanghe, Changjiang and the Lancang Jiang. The SNNR was established in 2003 by the Chinese authorities to alleviate environmental deterioration with particular focus on wetland, wildlife and forest and shrubland protection (FFI, 2004). Fig. 3.1 shows that the government has implemented a three zone system for the reserve divided into core, buffer and multiple-use experimental zones. The SNNR is the largest nature reserve in China and has an area of about $363,000 \mathrm{~km}^{2}$ (about $50.4 \%$ of Qinghai). Initial plans for the establishment of the reserve focussed on a complete resettlement of its 200.000 local inhabitants by 2011 . A representative of FFI explains:

"Sanjiangyuan was suddenly "discovered" as a national nature treasure by the Chinese authorities some years ago. It was assumed that due to the impacts of climate change and the regional significance of a healthy ecological state of the Tibetan Plateau, immediate action was required. However, the government overreacted with its resettlement plans for local people. Some scientists in Beijing even believe that the Tibetan Plateau should be completely emptied of local inhabitants." 9

\footnotetext{
${ }^{9}$ Indeed, resettlement programs have already been realized in some townships and I have seen some of the resettlement housing blocks while visiting the region
} 


\section{Sanjiangyuan National Nature Reserve, Qinghai, PRC}

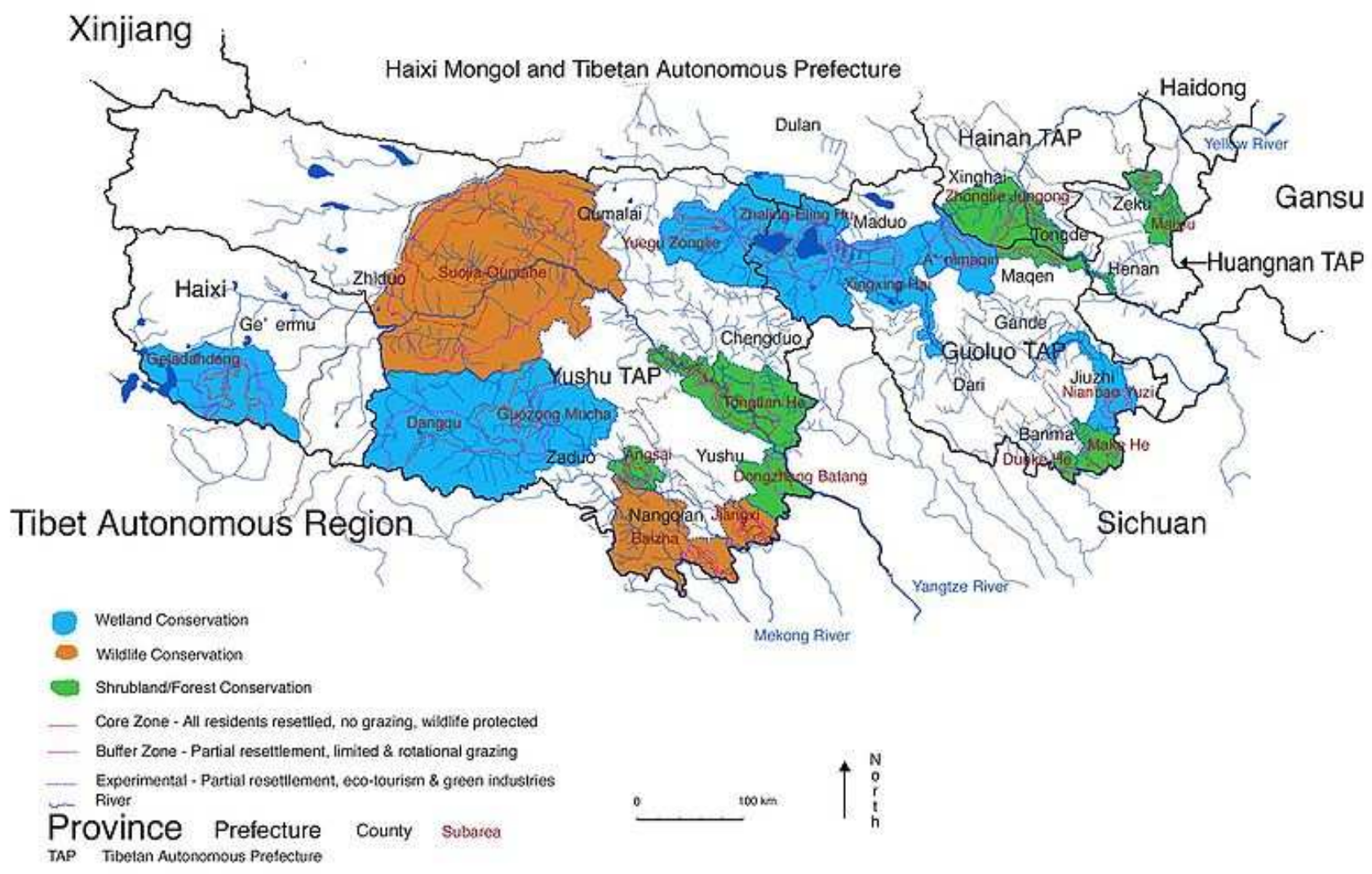

Figure 4.1 Map of Sanjiangyuan National Nature Reserve with conservation subareas and zoning system (Adapted from: http://commons.wikimedia.org/wiki/File:SNNR_Map.jpg)

It is apparent that there is much dissatisfaction among the non-governmental community regarding government conservation policies. My interview partner from the Bridge Fund, an international development NGO that is working exclusively on the Tibetan Plateau, mentioned that there is much frustration owing to a significant gap between the official government conservation policy and its implementation on the ground:

"The Chinese government still struggles to realize the shortcomings of a top-down conservation approach for the Tibetan Plateau region. Thus, despite an increasing official rhetoric of 'bottom-up' approaches, conservation on the Plateau is remarkably 'top-down'! This has a marginalizing effect on traditional resource management institutions and on the preferences of local communities, especially in view of protected area management." 
The interview partner from Fauna \& Flora International (FFI), an international conservation NGO, further explains that government approaches towards conservation in the region have been controversial in various aspects:

"Science-based, top-down decisions that are made in Beijing will not work and can have disastrous effects on local people and their environment. After the invasion of Tibet by China, people were encouraged to move to the town. Then after the introduction of the household responsibility system, they were encouraged to move back to pasture communities and land was distributed into individual household plots. However, the fencing of grassland is partially responsible for ecological degradation and has an impact on wildlife movement. Controversially, local herding practices were stigmatized as being harmful to the environment. Due to an overall growth in population, there are now more families than in the past. This has resulted in an increase in the number of livestock herds rather than an increase in the size of livestock units which more or less remained the same size."

The first main theme that surfaced in my interviews with NGOs active in the region was that there is no one blueprint approach for biodiversity conservation on the Tibetan Plateau. Instead, interviewees emphasized that the framework for a conservation project tends to be defined by local realities on the ground and is adjusted throughout the working process. Research participants pointed out that the Tibetan cultural realm is much more diverse on the ground than it appears on the surface. This diversity requires considerate project planning in advance. As one interviewee from Shan Shui, a Chinese conservation NGO that is affiliated with the Peking University, noted,

"The relationship between culture and conservation, community knowledge and conservation initiatives has to be understood before setting out a conservation framework for the area of interest. Tibetan culture is diverse in itself and a great degree of cultural sensitivity is needed when approaching conservation stakeholders on the ground. There are three different main Tibetan languages and cultural areas, Lhasa, Amdo, Kham, and Tibet's diversity has to be taken into account in any project's activities. However, China's conservation politics are affected by the centralized character of the government, and government authorities and external partners often struggle to recognize this diversity and do not know how to approach a diverse community landscape as it is found on the Tibetan Plateau." 
This argument was supported by the interview partner from the China Exploration and Research Society (CERS), a Hong Kong based NGO which focuses on the relationship between traditional minority culture and the natural environment:

"In the early days of CERS, there were many different, often conflicting perspectives about the protection and use of natural resources between government, scientists, NGOs and local communities. There are difficulties to identify local problems and needs at the central or regional government level. Chinese politics are characterized by a centralized government and decisions about biodiversity conservation on the Tibetan Plateau and any other regions are made by high officials in Beijing. This not only undermines the diversity of the region with different lifestyles among the population but also the different degrees to which people are affected by conservation approaches."

In addition to socio-cultural variations on the ground, there exist differences in the relationship between local people and government authorities. For example, as the interviewee from FFI stated, "Different cultures in different natural environments have experienced a diverse conservation history and relationship with government authorities. For this reason, a conservation approach in one region might not work in another and a diverse range of project development patterns is required."

In response to this diversity, Future Generations China, the Chinese offset of Future Generations, an international conservation and development NGO based in the U.S., applies a seed-scale approach for the selection of project communities:

"Different programs are implemented in different communities. Human influence on the environment is a major selection criterion. Communities are selected close to the area of interest, for example nearby nature preserves, where there is a high degree of mutual influence between humans and the environment, or a close human-nature relationship. Our organization assesses the potential of growth for projects within the community and provides expert inputs to local initiatives. We try to incorporate whoever is available within the community to create a commonsense of partnership. If a project proves to be successful in one case, we try to implement it in other locations."

NGOs have not only recognized the need for more adaptive conservation approaches for the Tibetan Plateau region, they also centre their approaches on the participation of local resource users. The following section outlines NGOs' influence on the transformation of local conservation governance towards more collaborative approaches. 


\subsubsection{NGO approaches towards negotiating conservation governance arrangements}

\section{Approaches towards co-management}

NGOs play a major role in the development of more inclusive and cooperative conservation approaches on the Tibetan Plateau. All interview partners affirmed that the cooperation with local communities in conservation projects is indispensable. The degree of community involvement in conservation, however, varies among the different organizations and their project activities on the Tibetan Plateau. There are various examples of community participation or co-management agreements in conservation projects. Some projects, have a clear focus on community leadership in conservation, others involve the local community mainly in the implementation process and in related activities. The representative of Plateau Perspectives, an international NGO that is working on conservation and rural development in Yushu Prefecture, explains that the Tibetan Plateau currently serves as a playground for the experimentation of different conservation approaches:

"The overlapping areas of authority and responsibility shared between different stakeholder groups are presently being defined through experimentation with different models of community involvement in conservation and management of protected areas in the region. In co-management, community is more involved in the 'implementation' aspects of conservation work such as wildlife monitoring, anti-poaching, and environmental awareness building with the nature reserve field station in a coordinating and overseeing role. In the conservation contract model, more authority in decision-making and execution, is given to the local community, with the government management of the nature reserve managed being more of an evaluation role."

In addition, the interviewee from Plateau Perspectives highlighted the contribution of community conservation initiatives to regional conservation achievements:

"Several innovative community-led initiatives have begun over the past decade in the headwaters, or source area, of the Yangtze River - from the establishment of a local grassroots NGO, to a community co-management partnership within a national nature reserve, to the creation of a herders cooperative that assists local people to mobilize their efforts and to coordinate economic development activities in rural grassland areas." 
Plateau Perspectives is the main international partner of the Upper Yangtze Organisation (UYO). UYO was the first grassroots Tibetan NGO in China, founded by local Tibetan herders in 1998. In the same year, the Upper Yangtze Organisation (UYO) introduced the concept of Local Protected Areas to the Suojia township in Sanjiangyuan with the assistance of Plateau Perspectives. On behalf of the interests of local herders, a multiple-use land management plan was designed to protect local wildlife and ecosystems, in particular the snow leopard, the Tibetan antelope, the Tibetan wild ass, the black-necked crane, the wild yak, and a large wetland (Foggin, 2005).

Under the supervision of local authorities, an Ecological Protection Monitoring Committee was set up consisting of local herdsmen who conducted regular patrolling and monitoring in the Local Protected Areas, this was integrated with their daily activities such as tending grazing herds. Since the establishment of the Ecological Protection Monitoring Committee, regulations and contracts on biodiversity conservation have been agreed upon with the different communities (FFI, 2004). It was the concept of Ecological Protection Monitoring Committees that lay the foundation for future projects in the region.

Plateau Perspectives has been working in the Suojia township since 1998, and to a lesser extent also Qumahe township (Cuochi village), all in Yushu Prefecture, since 2001. After the establishment of the SNNR, the organization initiated the Suojia Community Co-management \& Biodiversity Protection Project as a mutual collaboration project with government agencies, local civil society and local communities. The policy framework along with some financial support, are provided by the management bureau of SNNR and local authorities. Plateau Perspectives, in cooperation with the UYO, contributes technical, logistical and financial inputs (Foggin, 2005). The representative from Plateau Perspectives gives a more detailed account on how communities are involved in the conservation projects:

"Community approaches can be done both through formal processes for example workshops where many agencies or stakeholders are invited, and through long-term experience and relations with local community and government. Our work is entirely built upon close partnerships with local people and authorities at different government levels. Our projects are based on a network of community conservation wardens consisting of a group of local herders, whose main responsibilities are to manage and protect ecologically important habitats, to carry out anti-poaching patrols and to regularly monitor wildlife populations. In practice, our local partner organization 
[Upper Yangtze Organisation] and the local government decided that village leaders would serve as the monitors since they generally have a higher level of education, and travel more than other people in their communities. Also, as democratically elected leaders, they are generally held in high esteem within the community."

The participatory approach introduced in the initial formulation of a regional land-use plan for Suojia was unique in the way it scaled up the participation of local people at a regional level, across several villages, and engaged with them to consider the actual content of projects and plans, not simply the selection of which person would implement conservation policy designed by central authorities in Beijing (Foggin, 2005).

Plateau Perspectives and FFI have both been working in Sanjiangyuan with local herders and local grassroots organizations on the co-management of natural resources since well before the Chinese government began its plans of establishing a national nature reserve in the region. Similar to Plateau Perspectives, FFI has a project focus on co-management and views local people as main the stakeholders in conservation. The representative from FFI illustrated by the example of the Sanjiangyuan National Nature Reserve (SNNR) the complicated governance arrangements behind biodiversity conservation on the Tibetan Plateau and NGOs' role in negotiating these arrangements in favour of local communities.

The representative explained a major challenge in convincing the government about the benefits of co-management was the fact that various government departments were involved in the conservation process in Sanjiangyuan. These departments included the Qinghai Environment Protection Bureau, the Qinghai Forestry Bureau (QFB), the Management Bureau of the nature reserve which is under the immediate administration of QFB, and several administrative organizations at prefecture and county levels such as Animal Husbandry, Forestry \& Environment Protection Bureaus and township governments. A positive development in 2001 was the combining of the environmental protection, agriculture, animal husbandry and forestry bureaus at prefecture and county which clarified the management relationships between departments (FFI, 2004). The organization's objective of producing a co-management plan was to bring all the stakeholders together to build public and government awareness surrounding the conservation of biodiversity and the threats to its existence.

"The Environmental Protection Bureau set up an eco-functional zone at Sanjiangyuan which later became the nature reserve. Before the establishment of Sanjiangyuan, the 
community presented a list of ideas to FFI. We then approached the Environmental Protection Bureau and Forestry Bureau with the project plan of a community-based reserve. After the Chinese government founded the reserve's management bureau, there were concerns within the bureau how to run the nature reserve effectively despite the deficiencies in human and financial resources. As a result of the co-management project the residents of the project location were able to draw the attention of government experts to their commitment to environmental protection and wildlife conservation and to introduce them to the concept of co-management."

The participation of communities in natural resource management and conservation was a new approach in the project area. Under the project, FFI created local Environmental Monitoring Groups with local people as environmental stewards that were supervised by the UYO. The local community was represented by the UYO throughout the management process. The interviewee from FFI is confident about the project's success:

"The Forestry Bureau retrospectively recognized the community reserves and employed local people. Co-management is now a main management approach within the reserve with conservation initiatives based on traditional institutions continuing with outside support at the same time. Other achievements include the adoption of local village-level regulations and local contracts for biodiversity conservation."

The interviews showed that all NGOs encourage consultation and cooperation with local people and advocate for local leadership and not only just involvement in conservation projects. Community leadership is also a main objective in the projects of Future Generations and has made community-based conservation its main project approach. Future Generations started its conservation activities in 1992 with the Qomolangma ${ }^{10}$ Nature Preserve project in the Tibet Autonomous Region. It is the region's oldest 'mega preserve' and was established in 1988 by the government of the Tibet Autonomous Region as a provincial level nature preserve to protect the fragile environment around the Mount Everest. Later in 1994 the State Council upgraded it to a national level nature preserve.

Conservation management in the Qomolangma National Nature Preserve (QNNP) is separated into three types of land use: a core zone where natural resource extraction is discouraged, a buffer zone that allows sustainable resource use, and a development zone that allows more intensive land use (Fleming et al., 2007). Future Generations successfully

\footnotetext{
${ }^{10}$ Qomolangma is the official transcription of the Tibetan name for the Mt. Everest
} 
convinced the government to employ local people as preserve wardens. As a result, the QNNP, recognized in 1999 by the United Nations as one of the world's most successful examples of sustainable development, was the first major protected area in the world to be managed by local people. The interviewee from Future Generations explains the main project objectives:

"It was one of the first nature preserves to be managed by local volunteers under existing government structures. The preserve focuses on a holistic approach to management that integrates economic, social and environmental concerns into the conservation management structure to bring local communities back into the conservation process and simultaneously addresses their development needs. It allows human habitation within the park boundaries and the designated mixed land use zones. "

The government cooperates with Future Generations to train community members to take on environmental responsibilities. Local communities are trained in developing monitoring and feedback mechanisms to make use of natural resources while at the same time meet the national conservation targets (Fleming et al., 2007). The organization recognizes the importance of feedback from communities and local authorities:

“The Pendeba project selects and trains local conservation leaders [Pendebas] in each community. The training sessions in conservation work, basic healthcare and economic development issues last for about four to five days. These training sessions further serve as an opportunity for mutual sharing of experience from previous years. The Pendeba project has a one year evaluation circle and people are invited back for further training."

This close partnership between local people and the government has proved to be a great success. In 1994 the QNNP started with 14 Pendebas, now there are more than 200. The NGO cooperates closely with local authorities not only to gain permission for project activities but to assist them in addressing conservation related issues, as the interviewee from Future Generations explains:

"Government authorities often have difficulties with identifying local problems and needs. Smaller NGOs like our organisation are more flexible in approaches

\footnotetext{
${ }^{11}$ Pendeba is Tibetan and means "Worker who benefits the village"
} 
as we have a direct link to communities. We assist local authorities in arranging programs and addressing local interests. Besides, we promote new ideas and educate local stakeholders about the objectives behind the projects. Local co-ordinators from our organization are responsible for the organization of activities which are supported by local and outside expertise, local government departments and universities."

The QNNP is a leading example of the benefits of cooperation between government authorities, NGOs and local communities. Implemented in one of China's poorest regions, the project has led to significant improvements in local livelihoods such as better health care and more income opportunities. More important for conservation, it has contributed to a significant rehabilitation of wildlife populations (Fleming et al., 2007).

While in the examples mentioned above conservation arrangements mainly resulted from the interplay between government, NGO and community interests, some NGOs such as Friend of Rural Community Development (FRCD), a local grassroots NGO based in Xining whose focus is on rural development, apply a bottom-up approach where the community defines the problems:

"Our organization advocates an approach where the community comes to the organization with the problem. The problem is then evaluated by the NGO, followed by a resource capacity assessment of our organization. If we think we can provide a solution to the problem, we develop a project proposal for the community and seek donors depending on their funding interests. As a NGO, we mediate between communities, donors and the government. After funding is found, local communities and government are informed. Usually, the lowest responsible government authorities at the community level are approached with the project ideas e.g. in case of environmental projects to the local Environmental Protection Bureau. Local authorities then take over the authority and management of the project."

A contract between the village and the local government that clearly defines the role of locals in the project is signed followed by a contract with the donors. The NGO reserves the right for project supervision and inspection of quality of standards e.g. concerning school buildings provided by the NGO. So far, the organization's environmentally focussed work has concentrated on the provision of environmental solutions and training to communities. For example, FRCD introduced solar cookers to rural households to cut the use of firewood. 
"We started with the solar cookers project as a model project. The necessary facilities were provided to a selection of around five households per community that were interested in the project. These model households eventually convinced others about the benefits of a solar cooker. Solar cookers are easy to use, are clean and convenient and about $50 \%$ of the communities can afford it. The project was so successful that in some counties nearly every household now has a solar cooker. Research that was conducted on the social, economic and environmental impacts of solar cookers revealed a significant reduction in the use of firewood and in the time needed for collecting it."

In cases when projects take place at the individual household level, local authorities do not need to be approached. At the time of the interview, FRCD was planning the introduction of community-based conservation projects. Their plan was to set up community-based environmental committees. Based on a conservation contract model, decision-making authority will be delegated to local community members.

Community-based protected areas based on the conservation contract model, is also the main project approach of Shan Shui. Shan Shui has a focus on biodiversity hotspots in southwest China and on the Tibetan Plateau. This focus has emerged from Shan Shui's past working experience as a partner of Conservation International. The interview partner of Shan Shui pointed out the benefits of conservation agreements between communities and the government:

"The major question for biodiversity conservation in the region is how to improve conservation governance. Government projects such as the rigid establishment of nature reserves are usually not the economic way of conservation. There is a need for community initiatives and mutual exchange which has advantages on both sides. Conservation agreements between government and community are a first step towards reconciling different conservation objectives."

Under "Conservation Incentive Agreements" local communities (local resource users and the actual conservationists) and the government (the resource owner and de jure conservationist) sign an agreement which hands authority over to the community who take on a set of responsibilities as defined by both parties. The agreement outlines and allocates conservation tasks, benefit packages, payments, and rights. Conservation and administration rights will be authorized to a community committee. The committee organizes villagers to 
perform conservation management, is responsible for the formulation of natural resource use regulations with the community and works out compensation activities. An underlying assumption behind these agreements is the need for providing incentives for conservation approaches to be accepted by the community. The establishment of a community conservation fund encourages community conservation initiatives supported by a communication network that provides space for government, NGO and community interaction. The community's livelihood and culture receive government support in multiple areas such as education and livelihood improvement.

The literature points out the benefits of co-management as a knowledge partnership that gives access to various knowledge systems pertinent to the natural environment and encourages mutual learning (Berkes \& Folke, 2002, Berkes, 2009). There's a common agreement that the primarily science-based top-down decisions made in Beijing are neither economic nor efficient. In addition to creating community benefits, the representative from Shan Shui pointed out that the conservation agreement allows the government to align its own social development and conservation agenda with the priorities of conservation work. Still, the integration of traditional ecological knowledge and resource management institutions poses a greater challenge to conformist conservation approaches and ways to link theory with practice and science with policy.

\section{Approaches towards traditional community institutions}

The local population is without doubt the stakeholder group that is affected the most by conservation decisions. Not only do their livelihoods depend on a healthy environment but also the survival of their culture and traditions. Having lived a mainly nomadic or seminomadic way of life, a large percentage of people are not only affected by any changes in the ecosystem but also increasingly threatened by the forces of modernization, development and also by conservation policy itself. Intergenerational social and cultural change not only affects the traditional way of life but also results in a change in human environment relationships and behaviour among future generations (Kalland, 2000). For this reason, a major objective of my research was to investigate how traditional resource management institutions are currently approached in conservation projects.

CERS takes a research approach that takes into account local traditions and finds ways to bring different stakeholders together. The organisation's team is mainly composed of 
scientists that are trained in doing research about issues related to China's ethnic minority culture and the natural environment. The interviewee from CERS explained,

"The culture and livelihoods of many minority groups in China still depend on their natural resources. A main challenge is to make the government and other stakeholders understanding how community institutions are in many cases the most cost-effective and efficient way to conserve biodiversity."

This argument was supported by the representative of FFI who outlined FFI's efforts in assisting local organizations under its Tibetan community grassland project to build up trust among local resource users and maintain traditional institutions:

"In pure nomadic areas traditional institutions evolved around the "tribe". The "community" consists of up to 100 households that make common decisions about natural resource management, in particular concerning grassland and livestock management. We are working with local organizations and other groups to encourage grassland communities to form collaborative management committees based on their traditional form of social organization. These committees serve as a forum for discussions on conservation issues such as the predation of domestic stock by wildlife. The traditional Tibetan way of life does not have to be abandoned to protect the region's environment or to foster sustainable livelihoods. Traditional patterns of resource management are more adaptive to climate change, and more used to risk management than government policies."

Shan Shui addresses traditional resource management institutions by supporting sacred land conservation projects and advocates for a combination of traditional and modern conservation approaches.

"Traditional ecological knowledge is a tool that can link cultural diversity with biological diversity. Under our projects, we focus on useful knowledge from indigenous and modern societies. The government favours nature reserves as a typical modern approach to conservation. NGOs can help to combine both traditional and modern conservation methods and make modern methods acceptable to local people. Monitoring can be helpful to show the usefulness of local knowledge. Monitoring is nowadays introduced as "modern" technique but might already have been used in some ways by communities themselves for many years and has just not been recorded. We need to assist communities in recording useful knowledge as part of their cultural 
heritage. Communities might learn to adapt to new circumstances but we need to learn more about their culture and the religious and spiritual connotations of knowledge need to be considered. To update our own skills, we organize scientific expeditions with team members from various research institutes, government levels, and NGOs and hold training sessions on stakeholder coordination."

In 2007, Shan Shui started the project "Through their eyes" that trained local people in the rural areas of Yunnan, Tibet and Qinghai in making documentaries about environmental protection. The aim of this project was, based on the perspective of local indigenous people, to document the interaction between local traditional culture and environmental protection, the conflicts and changes local inhabitants feel confronted with as well as participatory forms of discussion (Homepage Shanshui).

Movements to more inclusive conservation approaches that honour local knowledge and support traditional institutions can especially be attributed to a number of local pioneer organizations or individual pioneer thinkers that build the bridge between local traditions and the modern world, as the interviewee from the Bridge Fund notes:

"Guanxi [network of personal relationships] and local networks are essential for NGOs working in the Chinese conservation context. In the Tibetan herding context, local grassroots NGOs help to give local resource management institutions a voice and ensure that the local herder community is not seen as a problem but as part of the solution.”

Even when NGOs, both national and international, have been involved in, and promoted, a fuller inclusion of local communities and traditional institutions in conservation and the sustainable use of natural resources in the Tibetan plateau region, the progress has been slow. A spokesperson from Plateau Perspectives explains:

"Traditional resource management institutions are formally not much addressed. Successful inclusion of traditional institutions needs government support, which is now partially forth-coming. But proper integration at regional scale needs careful thought, not simply an edict." The interview partner also stressed the importance of changes in human-nature relationships: "A sense of being closely dependant on nature has led people to want to protect the wildlife of the region; but some of this interest or desire is also now eroding as human-wildlife conflicts increase." 
The increasing impact of novel, outside factors such as climate change, urbanization and tourism development further challenge this. The representative from Shan Shui puts it this way: "The own knowledge of communities on sustainable resource management might in view these factors not be enough and needs a back up from outside. However, little is known about local traditional resource management institutions and we actually don't know how communities are able to cope with these new challenges."

NGOs are increasingly becoming conscious of the change in local socio-cultural structures and the representative from Future Generations explains what challenges this creates for conservation:

"The preservation of grasslands and conservation of natural resources is important for the survival of Tibetan culture identity but the effects of changing settlement patterns on traditional livelihoods are continuously spreading. There are various underlying reasons for natural resource degradation that have to be identified and some even date back to centuries."

Biodiversity conservation is related to a wide spectrum of activities in view of traditional natural resource management. Local land use is one of the most important factors affecting grassland biodiversity but scientific ambiguity exists on its impact. The representative from Shan Shui mentioned that knowledge and scientific data related to the region's ecosystems and socio-ecological systems remains incomplete despite ongoing research by Chinese and international scientists. This is further challenged by an incomplete understanding of community knowledge about nature and their impact on the environment:

"The factors that are influencing decision-making by local herders related to the use of natural resources come from several directions. The role of the NGO in this process is first of all an analysis of the traditional and non-traditional community conservation initiatives. Even if own cultural concepts about conservation exist on the ground, local communities also accept ideas coming from outside. To find better ways for conservation, government support is needed and local ability needs to be assessed. Finding the right way of negotiating interests with the government and communities prepares the ground for conservation projects and related activities."

Even if traditional natural resource management institutions are still on the verge of playing a major role in conservation projects, the preservation of traditional culture and 
knowledge has made some progress in other areas such as education and rural economic development. NGOs' attention for local traditional institutions, even if they not directly related to conservation, shows that NGO are determined to take a more holistic project approach towards conservation so as to build up trust at the community level.

Cultural preservation has become a major project activity of NGOs, mainly as part of projects related to the generation of economic income, as a representative from CANGO explains:

"Cultural aspects and knowledge of environmental issues are not much addressed so far but more progress has been made in approaching ethnic minority culture in aspects related to rural development. Projects that include community knowledge of ethnic minority groups focus on handcrafts production for encouraging local economic development and finding income alternatives while at the same time protecting their cultural heritage."

FRCD combines cultural preservation with its education projects. The organization's representative explains what further benefits this could have for environmental conservation:

"The knowledge of herders is not taught in schools and gets lost within one generation if people abandon their nomadic way of life. Immediate action is needed to maintain traditional ways of acquisition and communication of knowledge to other members of society. There is an increasing focus on sustainable education that includes environmental and cultural education within the non-governmental sector. Cultural preservation activities with focus on Tibetan traditional culture centre on the preservation of language. Folk tales for example contain a huge amount of knowledge and ethics related to the natural environment. In our cultural preservation projects, we focus on the collection of local proverbs that among others tell people how to interact with their social and natural environment."

In terms of what mechanisms can be used to revitalise and strengthen traditional knowledge and why communities rely on the support of external actors such as NGOs to preserve or strengthen their traditional institutions, the representative of FFI explains:

"We have helped to set up livelihood training centres for rural communities. Training focuses on handcraft production and stone-carving founded on traditional knowledge 
that is in danger of being lost to the younger generation. These skills can not only help herders diversify their sources of income but also help maintain their cultural heritage. Rural economic development is affected by supra-local market forces and communities lack the business know-how and organization to gain access to national or international markets. To assist communities in their economic development, our organization set up contacts with a NGO in Shanghai that is helping with selling local handcrafts in other parts of China."

The last examples shows that many of the activities related to the preservation of traditional resource management institutions are combined with rural development activities. Indeed, all interview partners have pointed out the importance of a holistic project approach for the Tibetan Plateau that combines conservation with the socio-economic development of project communities. 


\section{Reconciling conservation and development}

The reconciliation of conservation and development objectives is a complex issue in many conservation locations and in developing countries in particular (Zimmerer, 2006; Fisher et al. 2008). Environmental conservation and rural development have long been treated as separate sectors in China. More recently, sustainable development that preserves the fragile environment of the Tibetan Plateau and also creates prosperity for its population is outlined in many government agendas, NGO programmes and donor priorities. The challenge is to put this common objective into effect. The majority of my interview partners have expressed the importance of a holistic approach towards conservation that simultaneously addresses the development interests of local communities and guarantees cultural sustainability. For instance, the representative from Shan Shui notes,

"More knowledge is needed about Tibetan areas in various aspects. Some areas are without human inhabitants or have a small population; others show a more significant population. Different lifestyles can be found among the population and therefore communities are affected by conservation approaches by different degrees. The complicated development requirements on the ground often produce conflicts with conservation objectives."

When they first started out, a number of the NGOs that I interviewed more focussed on rural development issues such as poverty alleviation, health issues and primary education than being interested in environmental issues, partly because of the development priorities of local communities. However, environmentally focussed projects developed within the first years of their operation as they realized that only a holistic approach that encompasses considerate Initially CANGO focussed on poverty alleviation but now many projects are connected with environmental protection for example community vulnerability towards climate change:

"Projects have long time focussed on rural development and poverty alleviation. However, communities are showing an increasing awareness towards environmental issues. To support the development of adaptive capacity of communities and we organize funding support for local projects as for example tree planting activities against desertification." 
At the same time, ENGOs that who implemented projects primarily based on motivations for biodiversity conservation and on ecological selection criteria, also discovered the need for a holistic approach that simultaneously addresses social, ecological and economic concerns. In its initial conversation projects in the Tibetan Plateau region, FFI applied a species based approach with focus on endangered species and protection of their habitat by community reserves with communities as the main stakeholders. However, while implementing a project, other problems emerged within the project community that needed to be addressed first, as FFI's representative explains:

"While approaching communities with our ideas other problems showed up and local communities had different priorities concerning which problems needed to be addressed first. While local herders were sympathetic to wildlife conservation, they were also interested in improving their living standards, so discussions of conservation had to be coordinated with discussions of livelihoods development."

Social and economic development is unbalanced throughout the Tibetan Plateau region and the few development benefits are unequally distributed between rural and urban areas (Balla \& Qiu, 2006). Pastoral and traditionally nomadic communities often struggle with low levels of education and healthcare. All NGOs that I have talked to have now made the provision of alternative or supplementary models for economic development to local people a central part of their project activities and consider it a prerequisite for successful biodiversity conservation. They affirm that people nowadays are more open towards modernization and livelihood improvements and do not reject alternative patterns of development if it is for their benefit. Providing local communities with a new model of economic development is an integral part of the project work of Future Generations:

"As as a consequence of ecological and cultural transformation, traditional knowledge alone is no longer sufficient. Our Pendeba model has proved to be a highly successful approach for the active inclusion of communities in conservation and development projects and to balance local community development and local ecosystems. Pendebas provide their communities with education about environmental conservation, health issues and alternatives for economic income." 
Conservation touches upon critical social issues such as land rights, restricted access to natural resources and the availability of development alternatives. Besides the forced sedentarization policy of the government, an increasing percentage of nomad families voluntarily decide to settle down in urban centres. It is notable that a number of local grassroots NGOs concentrate their project activities on the vocational training of (former) nomad herders. The representative of FRCD mentioned that in the case of conservation of environmental resources, the provision of alternatives to rural communities and a diversification of income sources are essential:

"The selection of project communities depends on various aspects. An environmental selection criterion, for example the loss of forest cover, is often related to the economic development of rural communities. Communities in our solar cooker project location faced restricted access to natural resources after the introduction of strictly enforced government policies against the cutting of trees. As unrestricted access to resources such as wood was in this case too far from the homes of the community members, alternative resources were needed to redeem the loss of income. On the other hand, in regions that provide little natural resources, livelihoods mainly depend on paid work. In this case, income needs to be derived from other economic activities."

Adaptive governance recognizes the importance of creating incentives for people and economies to act in harmony with essential ecosystem processes and dynamics (Gunderson et al., (Eds.), 2010). Shan Shui created "Special Ecological Zones" to test out integrated conservation and development approaches. The interviewee from Shan Shui explains their function:

"We support using the market mechanism, and not allocating government funds, to encourage conservation action in China's rural areas. Conservation agreements are the basis for Special Ecological Zones. In Special Ecological Zones build on the foundation of the inherited traditional livelihoods in an area. We develop sources of income that derive from environmentally-oriented industries, including tourism, forest carbon sequestration, freshwater conservation, and community-based production of traditional goods. Special Ecological Zones serve as a concrete case study for regional policy planning and development and large-scale infrastructure projects, and as a standard for government review of its own ecological conservation programs." 
Putting the ecological and ecocentric motivations behind biodiversity conservation aside, the Plateau's various natural resources themselves remain an important source for the economic development of the region. Local products from the Plateau such as yak products, medical herbs or fabrics enjoy a high reputation throughout the country and are a favourite souvenir amongst Chinese tourists. Among these products it is one particular item that boosts the local economy - the caterpillar fungus (Winkler, 2008). Traders selling this important ingredient for traditional Chinese and Tibetan medicine can be found everywhere and many local people are involved in this highly profitable business. Speaking to a group of young people in Jiegu (tib.: Gyêgu), the main urban centre of Yushu Tibetan Autonomous Prefecture which was almost completely destroyed by the 7.0 magnitude earthquake in April 2010, I was informed that they and many others are now trying their luck in the caterpillar fungus business after the earthquake left them unemployed. 


\subsection{Conservation networks and stakeholder communication}

The previous part of this chapter has outlined different approaches towards more collaborative conservation arrangements on the Tibetan Plateau. By looking at the different conservation projects currently in place in the region, it is apparent that stakeholder diversity is a common feature, and that the interaction between different actors defines the framework for regional biodiversity conservation. Networks exist as structures of constraint and opportunity negotiated and reinforced between interacting individuals or groups of individuals (Kilduff \& Tsai, 2003). In the following subsections, I will investigate the key triggers and barriers to more collaborative conservation approaches by looking at the cooperation between government and NGOs, cooperation among members of the NGO community, and the cooperation between NGOs and local communities. In the last section of this chapter, I will outline the opportunities and challenges towards more cooperative approaches relating to the financial dimensions of conservation. In all sections I will explain how NGOs take advantage of the triggers or, conversely, approach the barriers to support local community institutions. I will conclude this part of the results with a brief summary of the main triggers and barriers to collaborative conservation approaches.

\subsubsection{Cooperation between government and NGOS}

The conservation network on the Tibetan Plateau has special characteristics that serve as windows of opportunity for public-private partnerships. The representative from the NGORC highlighted the role of NGOs in facilitating the formation of these partnerships:

"An important contribution of NGOs to the improvement of conservation governance is their effort in enhancing the communication between different stakeholder groups. This enables learning, facilitates the understanding of different positions and provides opportunities for negotiating different interests."

A major question for improving conservation governance is to investigate under what circumstances do NGOs and government cooperate and what network characteristics foster and hamper cooperation. This section will discuss the main triggers and barriers to cooperation between government and NGOs and their impact on conservation governance arrangements for the Tibetan Plateau region. 
Even though biodiversity conservation in China is faced with many institutional shortfalls at the policy level, NGOs agree on the necessity of working within existing government structures and not against them. The interviewee from NGORC explains:

"NGOs see their main function in raising attention to particular problems, providing solutions and presenting them to the government and affected communities. The government's attitude towards public-private cooperation on environmental issues is changing too. It now feels that it needs the assistance of NGOs and has created some legal basis for NGO-government cooperation. NGOs need to find out how to seize these opportunities for cooperation and need to understand the operational mode and procedures of the government and to achieve a balance of interests."

NGOs deal primarily with provincial and local level authorities. Of the NGOs that I have talked to, only CANGO and Shan Shui sustain a steady direct linkage to central government authorities through their special consultative status. CANGO was founded in 1992 and is registered with the Ministry of Civil Affairs. It is an umbrella organization for Chinese NGOs with currently over 130 members. The organization's focus is on the development of NGO \& government cooperation. CANGO also facilitates international linkages via its special consultative status with the UN and as a member of the IUCN network. Despite having a close working relationship with higher government levels, the interview partner from CANGO points out the importance of working with local authorities:

"Organisations need to discuss their plans with all stakeholders and especially need the support of local authorities. Community committee offices have their own development plans and so does the [central] government. As NGOs and local authorities both focus on local community development, they can learn from each other. Besides, NGOs can encourage the personal communication between local authorities and the public. Local authorities are evaluated for their performance and their use of the budget. Environmental protection is one performance indicator. NGOs can provide authorities with new ideas and encourage a move towards more cooperation on environmental issues."

It was pointed out in a number of the interviews that some level of personal relationship with government officials or a long working history of the NGO in the region are useful or even necessary for getting the government on board and for facilitating the cooperation between NGOs and the government. Shan Shui cooperates with the government at different 
institutional levels but benefits particularly from its advisory role within the central government:

"We advocate policies on the national and local levels to achieve eco-equity"

Scientific and policy support to authorities comes from our research centre based at Peking University. We demonstrate conservation methods and then try to sell it to the government. Our organization further provides advice on the drafting of conservation laws and has an advisory role at the People's Committee's Environmental Conservation and Social Development Centre. A reason for the close cooperation with the government is without doubt that Shan Shui's founder Prof. Lu Zhi had his own personal relationship with the government and is a member of the government's Scientific Committee. We further invite outside experts from China and abroad to support conservation knowledge. On the other hand, our projects require government support and we rely on making use of government data and information to identify conservation problems and strategies."

The interview partner from Future Generations explained that personnel relationships with government authorities also proved to be useful when Future Generations China started work in 1992 with the Qomolangma Nature Preserve Project in Tibet:

"The Qomolangma Nature Preserve was initiated by Dr. Daniel Taylor, the founder of Future Generations. He proposed the idea of establishing a nature preserve to officials in Beijing. Strong personal relationships of his family with local communities and authorities helped to gain support for the project. The project was further supported by the current Prime Minister Hu Jintao who was the Provincial Committee Secretary of the Tibetan Autonomous Region at that time."

It became evident from the interviews that the degree of cooperation with the government might vary at different institutional levels and among the different government agencies involved in biodiversity conservation. The introduction of more participatory forms of environmental governance is empirically easier at the local level where government staff may be more accountable and closer to the people most affected (Tyler, 2006). A representative

\footnotetext{
${ }^{12}$ Shan Shui believes that to conserve China's ecological environment we need to work with local communities to ensure that nature conservation brings them tangible benefits i.e. eco-equity.
} 
from FFI explained the difficulties the organization had with different government authorities during the co-management project in Sanjiangyuan:

"The conservation project was impacted negatively by a deep level of mistrust between some stakeholders from the NGO community and authorities at the local level. There was friction with the Environmental Protection Bureau about different conservation values and ideas in the beginning. The Qinghai Provincial Forestry Bureau had to be approached because of its responsibility for wildlife conservation but turned out to be less approachable than the management bureau of the reserve which seemed to be more open to new ideas. This was partly because it was staffed with locals that know better how to deal with local people."

To get the government on board, FFI cooperated with the UYO which had previous experience with coordinating among different government agencies. The interviewee mentioned that the local village and township government had a much closer relationship with communities than the provincial agencies. It was further helpful that some members of the local partner organization UYO have been working for the local authorities in the past.

Local governments may be closer to society but also more susceptible to the pressure of various interest groups (Sun \& Zhao, 2007). For instance, the representative from FRCD noted, "We sometimes receive different responses for our projects from different government levels. Local authorities are not necessarily more approachable than provincial authorities." The representative from NGORC supported this argument and explained that the understanding of and attitude towards conservation projects are often defined by local politics, leading not only to different responses at different institutional levels but in different fields of conservation work: "Cooperation with the government is easier in some areas than in others. Consensus is relatively easy to achieve in the area for environmental education. Other areas, for example, when NGOs expose the environmentally irresponsible practices of local governments, NGOs and the government are more likely to come into conflict."

Another common theme noted by interviewees was that NGOs when seeking cooperation with the government, NGOs should not move too fast to put pressure on authorities. Instead, NGOs have found that the preferred method is to gain the government's understanding through a step-by-step process, as for example explained by Shan Shui's representative: 
"The conservation process involves a period of adjustment and agreement at different stakeholder sites. During our projects we received different opinions about the projects at different government levels such as acceptance at provincial level but disagreement at the county or district level or the other way round. Our organization especially endeavours a good working relationship with local government bureaus. Government support at the local level is needed to ensure the stability of community organization and the sustainability of projects."

Despite positive developments in cooperation between NGOs and the government, it has to be taken into account that significant constraints remain at the government level in terms of NGO involvement in the decision making processes and the ability of NGOs to encourage more collaborative conservation efforts. One interviewee, representing an international NGO, explained it by asking: "Who is the biggest NGO in China or even in the world? The Chinese Communist Party (CCP). Founding its strength on the three pillars of mass organisation, public involvement and mobilisation, the CCP shares some characteristics with a NGO." The statement was intended to be ironic, and to emphasise that the Chinese government is too afraid of its authority to loosen the control over civil society development in the country and especially in a politically sensitive region such as Tibet. "In view of the immediacy of many environmental problems and the international attention on the Tibetan Plateau, the government expresses an increasing openness towards multiple solutions but remains the power [behind all] decision making."

At the same time, NGOs sometimes overestimate their contribution to the conservation process. As one interviewee from FFI noted, "In some cases NGOs take the credit for major conservation achievements and undermine role of the government. However, pioneer thinkers and conservationists can also be found at the government level. The bigger question for conservation governance in China is where and how to bring in communities."

To develop a better understanding of the processes behind the interaction between the government and the NGO community, it is important to understand the network dynamics among the different NGOs. The following section will explain the potential influence of these dynamics on regional conservation governance processes. 


\subsubsection{Networks within the NGO sector}

Conservation in the Tibetan Plateau region is not only confronted with a diverse ecological and socio-cultural environment, but also by a diverse range of NGOs and their projects. The international NGO community has been strongly involved in biodiversity conservation issues in the region for more than two decades and a number of international NGOs share a long working experience on conservation projects. During the last decade, conservation on the Tibetan Plateau has increasingly been supported by the involvement of local and national NGOs. Still, the majority of NGOs, both Chinese and international, operating in the Tibetan Plateau region can be classified as development NGOs working on general development issues rather than ENGOs that are specializing in environmental and conservation issues.

The literature describes the NGO network in China as rather badly organized and uncoordinated, with many NGOs not being officially registered and therefore missing out on opportunities to cooperate with the government (McBeth \& Leng, 2006; Gransow, 2009). CANGO addresses this problem by providing a platform for NGO development where information about NGO related regulations and policies can be shared for mutual exchange and learning:

"We provide training sessions for our members for example on participatory methods introduced by foreign experts and arrange NGO development seminars with local officials, officials from the civil affairs bureau, and at central level from the Ministry of Civil Affairs. We encourage grassroots democracy capacity building by stakeholder involvement based on the concepts of mediation and mutual learning. We further promote cooperation between business enterprises and NGOs and encourage enterprises to incorporate Corporate Social Responsibility strategies. On the other side, the NGO network is open to suggestions from other stakeholders. On behalf of the Ministry of Civil Affairs, we participate actively in international negotiations relevant to environmental issues."

During the interviews it became apparent how important a wide network of conservation experts and scientists from China and abroad is. Cooperation with national and international universities and research institutes is a common feature of all the NGOs I talked to. They agree that especially in view of scientific uncertainty, lack of information and official data on environmental change, a diverse range of conservation and development experts is needed to increase the chances of successful conservation approaches. The interviewee from Future 
Generations mentioned in this context the aspect of mutual learning: "We maintain a network of national and international conservation experts to provide communities with expertise on the local environment and on the other hand to learn what we can do to improve our conservation projects."

Mutual learning and exchange are especially important when a large and complex system of government administration leads to difficulties in disseminating acquired knowledge through different hierarchical levels (Tyler, 2006). As the interviewee from NGOREC explained, "NGOs can support vertical and horizontal information channels in various ways. NGOs with a good linkage to local and central government levels can provide higher government authorities with information which is not easy to obtain from local Environmental Protection Agencies."

A horizontal transfer of knowledge, (e.g. from herders to herders or local grassroots NGOs to communities), however, is considered to be less complicated (Tyler, 2006). Smaller NGOs see their strength in being more flexible in project approaches as they have a direct link to local communities. The example of FRCD is representative of many grassroots NGOs in China. Local grassroots NGOs such as FRCD are primarily composed of staff members coming from the project area themselves. They can easily assess the dimension of the required activities to achieve conservation objectives and translate them across the stakeholder network, as explained by FRCD's representative: "Our work is focussed on the household and community level. Coming from rural areas in Qinghai, we are familiar with the local situation and related problems and ways of thinking. This close relationship facilitates the interaction with local people and the introduction of new concepts to them."

However, locally-based NGOs are challenged by their limited staff capacity. Whereas international and some national NGOs are staffed with Chinese and foreign experts, local NGOs are mainly composed of a few staff members with local backgrounds and thus have a more limited resource base. FRCD for example has just three permanent staff members who are occasionally supported by volunteers and the "office" is based in the director's home.

In some cases, shortfalls in resources and capacity can be compensated by cooperating with other NGOs. For locally-based NGOs, linkages with national and international NGOs can be particularly critical. But also national and international organizations profit from cooperating with grassroots NGOs. One common characteristic of the national and international NGOs represented in this research have is that they work in partnership with one 
or several local grassroots organizations. The informant from Shan Shui explains the benefits of cooperating with local NGOs in terms of the transfer of knowledge:

"As a NGO based in cities, we encountered difficulties to keep regular communication with local communities in remote areas. To overcome this challenge, we developed a close partnership with local NGOs [...]. This approach has been proved to be a winwin model for both our organization and local partners. Local NGOs shared the responsibility to manage the grants in each region and own the rights to grant decision making. Capacity building was also provided to local partner NGOs to support their ability on project management. What's more, local NGOs developed their own community small grant program after the completion of the project."

The representative from Shan Shui revealed that the organization's shortfalls mainly lie in issues relating to rural development as their expertise is more linked to the biodiversity conservation aspect of projects.

“These shortfalls are addressed by working together with other organizations, mainly small local NGOs, which support our work in other fields such as education or economic development. An advantage of local grassroots NGOs is that they have already established a level of trust with the community. The use of existing networks, data, and experience makes it easier for a 'new' NGO to present their concepts to the community."

Cooperation and mutual support between NGOs also takes place at the international level. The Bridge Fund had an initial project focus on rural development but is now carrying out community-based conservation projects in cooperation with the World Wide Fund for Nature. However, the representative from the Bridge fund noted that it is the cooperation with local grassroots organizations that is essential for establishing partnerships within the Chinese conservation context.

At the same time, the history of some of the local NGOs operating on the Tibetan Plateau reveals how important links with international NGOs are in facilitating their establishment. Two critical aspects of these relationships are training for local NGO staff and the development of close stakeholder relationships with government authorities and donor society. For example, as a result of the success with the Pendeba Project of Future Generations, a local grassroots NGO named Pendeba Society was established to increase networking opportunities for project volunteers (Pendebas) in the Qomolangma Nature Preserve. The Pendeba Society 
is the first non-profit organization to be created in the Tibet Autonomous Region of China. The organization was established by a local Tibetan who worked with Future Generation's on the Pendeba Programme and completed a Master's Degree in Applied Community Change and Conservation from the Future Generations Graduate School in the USA. The organization applies a model of "Three-Way Partnership" that mobilizes the community to participate in the development of the protected area. This Three-way partnership involves "bottom-up" initiatives from communities, "top-down" support from government agencies, and "outsidein" training and support from organizations (Pendeba Society, 2010).

In addition to providing support in form of international expertise and funding, cooperation with international organisations further provides the international community with a broader picture about civil society development within China, as the representative from CANGO explains:

"Many NGOs in China have a long history but not much is known about it in the West. A common public opinion of strong government control over environmental issues also exists within China itself. However, the Chinese government is making progress in building more development space for NGOs through the introduction of new policies, moving to a more cooperative approach instead of control. For example, the government will pay for services provided by NGOs to the public. However, a problem is that many international NGOs think that China because of its recent economic growth is not a developing country anymore. On the other side, the Chinese government also does not want to show the bad site of the country's development.

Participation at international conferences is an opportunity to show a more comprehensive picture of China and draw the attention of the international community to China's NGO network and its activities."

While investigating the Chinese NGO network, it becomes evident that there are some specific characteristics of local NGOs and community features that are instrumental in conservation work and indispensable for its success. Various interview partners pointed out the importance of guanxi (informal relationships), networks, and pioneer thinking at the local level and how these lead to the formation of local grassroots organizations. Interview representatives from national and international organizations highlighted the efforts of the Upper Yangtze Organisation (UYO) in building the bridge between local communities and the wider conservation network. In some cases it is beneficial that key personnel of local NGOs come from the local government and that organizations are subordinated to specific 
government departments. This was also the case for UYO, as explained by the interviewee from FFI:

“UYO’s founder Zhaxi Duojie [Zhaduo] is widely known within the conservation network on the Tibetan Plateau. Zhaduo came out of the government and knows how to talk to people at various government levels. He is a good example of those local individuals that have an entrepreneurial spirit and pioneer way of thinking in terms of conservation approaches. He successfully convinced government officials about the need for including local people in conservation management. Zhaduo even tried to make foreigners feel "guilty" as they focus not enough on community benefits and care more about research based on western values and concepts."

However, an initial challenge for UYO was achieving legitimacy in the eyes of the government. Furthermore, it took time for people to understand how a local NGO could help them. Once local leaders came on board they conveyed the goals of the organization to local people and then membership increased among nomad families (Foggin, 2005).

The last two sections have shown that a good degree of cooperation with both government authorities and other NGOs is important for improving conservation governance in the Tibetan Plateau region. However, the sustainability of conservation projects heavily depends on the cooperation with local communities that are affected the most by conservation decisions (Naeem et al. 2009).

\subsubsection{Cooperation between NGOs and local communities}

In this section I will outline NGOs' efforts in establishing cooperative conservation partnerships with local communities. This will include detailing approaches towards mutual learning and dialogue, trust-building processes and methods for strengthening community intuitions.

Decisions are more widely accepted and supported if they are based on a common understanding of issues and stakeholders have the chance to voice their concerns (Prager, 2010). As the sustainability of conservation achievements relies heavily on local knowledge and understandings of conservation, NGOs play a major function in building environmental awareness, providing environmental education to local communities and thus, encouraging 
local participation. However, Chinese and Western perceptions about nature and wildlife sometimes differ - and so do the perceptions of local people. Consequentially, NGOs have a potential bridging function not only between different stakeholder groups but also between different cultures, ideas and concepts, as the interviewee from Shan Shui explains: "On the one side, knowledge at the grassroots level needs to be accepted and supported by higher government levels. On the other side, participatory methods and modern knowledge need to be introduced to and accepted by local communities."

In addition, as the representative from CANGO explains, sometimes it is more a question of one's own responsibility and what the individual can do rather than understanding the concepts of conservation: "NGOs major work in China is to promote public involvement and education about certain environmental issues that are usually discussed between government and scientists, for example issues related to species conservation or climate change, and what the individual can do about it."

Six out of nine interviewees emphasized the importance of trust building among the different stakeholders, especially among NGOs and local communities. To establish collaborative research and common conservation interests, outside actors also need to learn ways to negotiate with local peoples on how "our" research and interests can benefit "their" communities (Posey, 2002). Asking what criteria NGOs are adhering to for the selection of project locations or communities, the responses showed that this is a complex issue. For instance, the representative from Future Generations noted:

"It is important to identify the underlying causes of environmental degradation and biodiversity loss before implementing a project. These causes can be of a diverse nature and might at the first glance not directly relate to biodiversity conservation but need to be addressed to build the necessary level of trust with the community."

The interviewee explained that these causes often relate to problems in the rural development spectrum such as poor health care:

"As part of our Pendeba project, we trained local people in basic healthcare. There is often no access to basic healthcare facilities in remote rural areas and local people have to travel to urban centres to see a doctor. Local people usually don't have the money for travel expenses and for paying the doctor. In some cases, people try to get the money from selling wildlife products such as furs from wild animals." 
The importance of providing communities with clear conservation benefits as a means of trust building has been put forward by all interview partners. But what might be perceived as a community benefit by external actors might be of little importance to local people, as the experience of Plateau Perspectives shows. After a long discussion about how to best serve the community of their conservation project, the local government, Plateau Perspectives and its local partner organization agreed that education could play a central role in the community's development. However, when the issue was first raised with local herders, the idea of sending their children to school received mixed reactions (Foggin, 2005). The projects of FFI faced in their early phase various challenges on the local level too, as in the following explained by a representative from FFI:

"Initially, the project designed and provided capacity building training for local herders, but they did not understand the value or accept much of this. Some herders expressed the view that foreigners were only interested doing research for their own interests such as species conservation."

For this reason, the co-management planning process in Sanjiangyuan included activities such as setting up workshops to solve local herders' problems and build up their trust. An important first step was to make multiple stakeholders aware of the project to facilitate the involvement of wider stakeholder groups. A workshop with relevant stakeholder groups was set up to provide training to local herders and lay the foundation for the creation of local protected areas. In addition, a festival was organized in Yushu to convey the importance of conservation to local people and to get them on board.

"The community reserve concept received good reception from local people. They supported the need for the protection of wildlife, partially based on their strong belief system. There was also a strong involvement of local monasteries that have already been working together with the government on other issues and established close working relationships. Local monasteries further have a strong influence on local communities which was helpful for convincing them about the benefits of conservation."

Based on the experience of the NGOs I interviewed who work on conservation issues, biodiversity conservation introduced as an outside concept is supported by a local responsibility for land founded on a strong spirituality of the people and related ecocentric 
environmental ethics. However, NGOs are also aware of the ongoing socio-cultural change in the region and what challenges this might create for the sustainability of conservation projects.

CERS implements projects that build social cohesion and mobilize communities through local action, as explained by the organization's representative:

"The Tibetan context shows that many traditions such as horse riding festivals are kept alive as a part of shared cultural identity. Keeping the community sense alive can be done through projects focussed on traditional culture, exchange of values and the concept of socio-ecological sustainability. We supported for example a local archery festival in Yunnan. Whereas old people still remembered the crossbow skills, younger generations had to be taught how to use a crossbow. The festival as an integrated community activity not only supported tourism development within the community but also welded local people together and supported their traditions."

Building trust and the expansion of social networks are closely related to investments in social capital (Folke et al., 2005). Apart from relations of trust, social capital includes reciprocity, common rules, norms, sanctions, and connectedness in institutions (Pretty \& Ward, 2001). The internal weakness of communities may be complemented by exchange with other communities. The representative from Shan Shui outlines the important function of horizontal linkages among their project communities:

"To promote community conservation capacity and conservation effectiveness at the grassroots level, it is also important to promote the sharing of experience among the different project communities. There are similarities among different regions and in lessoned learned from conservation projects. Community exchange and sharing has been proved to be a good approach to enhance capacity and increase conservation knowledge. On the other side, communities need to develop a feeling and understanding for science, and there is no need to make them feel strange about it."

Apart from workshops, conferences are a popular occasion to bring different stakeholder groups together. The representative from CANGO explains the benefits:

"Conferences are organized under the concept of action learning and open space and involve apart from NGOs local authorities, ministries, media, enterprises, research institutes and student groups. They are a good opportunity for local communities and authorities that lack knowledge and expertise to exchange knowledge with 
conservation experts. But more important for our members is the expansion of their own network and receiving suggestions from the network how to address conservation issues."

Promoting dialogue between different interest groups requires the development of codes of conduct, standards of practice, and principles for equitable partnerships (Posey, 2002). It is worth noting that interviewees highlighted the importance of creating 'partnerships' with local stakeholder groups, as the representative from Future Generations explains: "Local scepticism challenges the support of outside conservation concepts and with already having the government playing a dominant role, it is important that the NGO is being regarded as a partner and not instructor and that mutual learning is encouraged."

However, partnerships with external organizations, whether it is with government agencies or the non-governmental conservation community, are not always beneficial to local communities. Problems partly result from diverse interpretations of the concept of participation. Participation can be understood as involving people in all stages of project planning, implementation and evaluation but it can also be used by external agents as a tool for utilitarian purposes such as mobilizing the masses for predefined purposes (Parfitt, 2004; Leal, 2007). The representative from Shan Shui explains what consequences this has for the inclusion of local traditional resource management institutions:

"There is a slow trend towards more inclusive approaches that empower local people and their resource management institutions. Institutional support is still lacking and multidisciplinary and multicultural responses are needed. It is assumed that most communities lack the capacity and opportunities to make cautious development and conservation decisions. In addition, the Chinese government and outside experts usually make such decisions, excluding local communities from important decisions that directly affect them and their natural resources."

In the same way, the concepts of local ownership and partnership can be interpreted and applied in different ways. "One danger for external organizations is that (knowingly or unknowingly) they appropriate for themselves ownership of work that in fact is the intellectual property of other people, often the local or indigenous people. In Suojia, this problem has already occurred on at least two occasions when external agencies acted as though the community had undertaken no previous work of its own and all the efforts made by the community were futile without the external assistance" (Foggin, 2005, p.146-147). 
In addition to problems related to community participation, external partners have a considerable impact on conservation projects through funding related rules and interests which will be described in the following section.

\subsubsection{Financial dimensions of conservation and relationships with the donor society}

The availability and management of resources, in particular funding, puts a major constraint on the implementation of conservation projects on the Tibetan Plateau. Being less affected by the national economic boom than China's eastern and south-eastern provinces, financial support is essential for the general development of the Tibetan Plateau region and realizing conservation objectives. Funding was named by all interview partners as a major challenge for operating efficiently and for achieving conservation objectives and sustainable development. In this section, I will describe the opportunities and challenges related to the financial dimensions of conservation. I will describe how these influence the relationships between different stakeholders and their potential impact on conservation governance arrangements.

A thorough assessment of the operation costs and the feasibility of projects are crucial in view of the limited capacity of NGOs. As is the case for most grassroots NGOs, the representative from FRCD noted that the organization's operation is highly dependent on external financial support: "A major role of NGOs is the provision of funding to local communities. Very often we have to turn down project proposals when we realize that our organization does not have the capacity and enough financial resources to implement a project." FRCD is planning the introduction of solar heaters to further reduce the amount of firewood needed for heating rooms. The interviewee from FRCD explained that as solar cookers are outside of the building, coal is still needed for heating the rooms. Besides, solar cookers can only be used during daytime. Whereas solar cookers are quite affordable at 20 US\$, solar heaters will cost about 300US\$ which a normal household cannot afford. The organization also plans to provide communities with biogas systems but is reliant on finding experts for the project implementation and funding resources. Considering the low capacity in view of human and financial resources, small NGOs often have no other choice than to focus on small-scale projects within selected communities. As a result of financial and human resource limitations, large scale conservation projects are mainly in the hands of big 
international NGOs or coordinated between different international NGOs and the Chinese government.

In some cases it is more the accessibility of funding sources than their availability that causes difficulties for NGOs, as explained by the interviewee from CANGO:

"Funding is a major problem for NGOs which is often supported by international NGOs. Local grassroots NGOs often have difficulties in gaining access to these funds. By taking fundraising as a major field of our work, we try to connect these NGOs to different funding channels."

The representative from Shan Shui supported this argument and noted:

"Operational costs have to be calculated and the availability of support from various sides has to be ensured to put a project into effect. In addition, funding for non-conservation related activities such as health and education care increases the costs of projects. However, Tibetan areas now attract more interest, both national and international, for conservation and hundreds of grants are available for the region. In order to gain access to these financial resources it is important to establish networks."

In addition to the operational costs related to conservation projects, NGOs and authorities need to work out systems that compensate villagers for the loss of domestic stock from wild predators and other costs related to human wildlife conflicts (Fleming et al., 2007; Foggin 2008).

The interview partner from international NGOs noted that it is for a large part the availability of international financial resources that motivates government action for conservation. For instance, the interviewee from FFI explains,

"The Chinese government even set up its own NGOs or 'GONGOS' to attract international funding for conservation projects. One of the best examples is the China Wildlife Conservation Association that was established initially to attract funding for the conservation of the Giant Panda. In addition to international financial resources, funding needs for conservation are increasingly met from in-country assistance and supported by government assistance in regional infrastructural development." 
Besides cooperation with government authorities and other NGOs, networks with business enterprises are increasingly supporting civil society movements by financial and technical means. The NGOs I surveyed also revealed that money for particular projects has increasingly been provided by national and foreign enterprises over the last years as part of the corporate social responsibility agenda or because of personal interest. As, the representative from Shan Shui noted, especially projects that focus on the protection of flagship species and their habitat such as the Panda attract huge amounts of money. Lufthansa for example, supports biodiversity conservation projects in China for cranes, the company's emblem, which have their main habitat in China's western provinces. Apart from this, business enterprises can have a lobbying function as well and convince the government of the necessity of action by providing funding. State-business relationships in China tend to be more intimate than in western capitalist countries, and the absence of a basic consensus on issues opens up opportunities for negotiation and bargaining until consensus is reached (McBeath \& Leng, 2006).

However, NGOs' experience with the donor society is mixed. This is partly due to a diverse range of actors within this stakeholder group. Foreign funding partners have their own individual history of establishment, have particular connections to the state or to political institutions and thus could be each in their own way politically or financially bound (Sausmikat, 2009). As an interviewee from CERS explains,

"The donor community is a mix of smaller and bigger enterprises, and different government levels such as large-scale development aid or foreign embassies." Although donors are critical to ensuring NGO operations in most cases, some donor priorities can also limit NGO activities.

For example, as the same interviewee from CERS noted, "In our experience individual donors are more flexible than for example governments or multilateral organizations that often have fixed agreements on the distribution of expenditures." These fixed agreements on expenditures cause dissatisfaction among both NGOs and communities, as explained by the interviewee from Shan Shui:

"NGOs have their own, individual strategies, some just work at community level, some in a wider network influenced by the individual interests of NGOs and donors. In the latter case, NGOs often have to adjust their project proposals to the changing interests of donors. Some of our main funding partners had a 
primary interest in economic development, now they show more consideration for environmental issues."

NGOs welcome an increasing diversification of funding interests among an increasingly diverse donor society but also point out the importance of providing community benefits. The representative from FRCD criticized that donor interests do not always benefit local communities:

"Money for our projects is provided by foreign embassies in Beijing, China-based and foreign enterprises and private donations. [...] Some donor projects such as waste management training for example for garbage removal from rivers, or reforestation projects improve the local environment and bring about clearly evident community benefits. For other projects that focus for example on the protection of wildlife species or their habitat, it is more difficult to explain what the benefits for local communities are."

Donor priorities and funding interests can be another reason why local culture and knowledge may only be touched upon on the surface in conservation projects. An interview partner of CERS raised the issue that many funding partners still focus too much on the conservation aspect of the ecosystem or species at issue and neglect the social dimensions of conservation:

"Donors often follow their individual interests and have pre-set priorities for funding before making a final decision about supporting a project. Some funding partners just focus on the wildlife and scientific aspects of conservation and the human dimension of conservation are less considered. Donors show too little respect and understanding for local traditions. Projects that build on local traditions such as 'environmental purification through incense sticks' sound for that reason less interesting to donors than the conservation of an endangered flagship species such as the Panda."

In addition, the interview partner of FFI noted another negative aspect of funding mechanisms via a criticism of how the competition for financial resources impacts the relationship among different stakeholders:

"There is a high competitiveness for funding, especially among big international conservation NGOs. However, these organizations are often very good in presenting, talking about problems and criticizing government but there is little action on the 
ground. NGOs often speak up for the different kind of endangered species but also local people need someone to speak up for them on the round table. “

As the interview partner further continued, donors are often not familiar with local realities and local partner organizations and on the other hand, local stakeholders are not familiar with the world of the donors:

"There is a high level of mistrust and misunderstanding among everyone: NGOs, government, donors and local people. Some donors treat Tibetan partners not good and try to put through fixed contracts because money has been peculated in the past. The donor world is very remote from local realities, leading to misunderstandings between donors and local partners. Donors are not familiar with and therefore don't support a bottom-up approach that lets communities pick NGOs and approach them with their problems. They have difficulties to understand community-based decision making process about projects and their priorities. However, projects will fail and a lot of money gets wasted if they are solely based on donor priorities and lack community support. "

The interview partner explained that some projects are difficult to attract funding as the donors show no understanding for them. In addition, the organization's Tibetan partners struggle to find their place in the donor world and foreign NGOs often have to play a role as translators that explain the donor world to Tibetan partners.

On the other side of the story, foreign organizations that want to work in China are confronted with a particular socio-political environment and sometimes do not know how to establish contacts and develop cooperation with local communities and Chinese NGOs (Sausmikat, 2009). The interviewee from Future Generations explains the difficulties that international NGOs working in China can have:

"The management skills of NGO employees, the unfamiliarity with the working environment as well as diverse local capacities create additional challenges for conservation projects. This is further complicated by the registration process for NGOs and a high degree of political sensitivity in the working environment. To decrease scepticism and increase the local adaptive capacity to new ideas, it is important that the NGO has to be understood as a partner that cooperates with the government and supports local participation." 
One of the interviewees explained that in view of the numerous conflicts and administrative efforts related to the funding of projects, a new approach of direct grants is currently being tested out with money going directly from donors to local communities and the NGO level being left level out. He is sceptical about whether this brings about a solution to the problem:

"Conservation is very much affected by a short-sightedness of some stakeholders. NGOs not only need to know their power and influence on other stakeholders but also how to use it efficiently. More important than testing out different channels of funding is to find ways to bring different stakeholders together and achieve a balance of interests."

\subsubsection{Summary of key triggers and barriers}

The results of my research illustrate a diverse range of opportunities and challenges related to the development of more collaborative conservation governance frameworks (see Appendix 1). An investigation of the key triggers reveals that the creation of partnerships, flexibility in conservation approaches, mutual learning, and trust building processes are seen as the most effective means to more collaborative conservation approaches. On the contrary, unequal power relationships, differences in the understanding of the concept of conservation and its governance and a diversity of interests and priorities are identified as the main barriers. The following chapter will provide a detailed discussion of the main triggers and barriers to conservation co-governance on the Tibetan Plateau in relation to the findings from the literature review. 


\section{Chapter V: Discussion}

\section{$\underline{5.1 \text { Introduction }}$}

This chapter provides a discussion of the major research findings in relation to the study's aim and objectives and the key themes that resulted from the literature review. I will discuss the key network characteristics that serve as triggers and barriers to more cooperative conservation approaches in favour of local resource management institutions. In particular, I will assess the role of NGOs as bridging organizations in taking advantage of these triggers or respectively approaching the barriers. Then, I will address the limitations of my research and how these might have impacted the results and give suggestions for future research. The chapter will conclude with a synthesis of NGOs role as bridging organizations in biodiversity conservation on the Tibetan Plateau and discuss the major steps that have to be undertaken to improve local conservation governance arrangements.

\subsection{Role of NGOs as bridging organizations}

The overall aim of my research was to investigate the potential role of NGOs as bridging organizations in the context of conservation governance in the Tibetan Plateau region. My objectives aimed to investigate (1) the different arrangements for conservation governance that are currently in place in the region, (2) the influence of NGOs on a movement towards more collaborative conservation approaches and (3) the main triggers and barriers to more collaborative conservation governance arrangements.

The literature review illustrated several key attributes of social networks and bridging organizations that have potential to influence conservation governance processes. Bodin $\&$ Crona (2009) argue that social networks can improve collaborative governance processes by facilitating the generation, acquisition and diffusion of different types of knowledge and information about the systems under management. I addition, significant variations in governance processes and outcomes can be expected among networks experiencing structural differences in terms of density of relations, degree of cohesiveness, subgroup interconnectivity, and the extent to which a network is centralized around one or few actors (Kilduff \& Tsai, 2003; Bodin \& Crona, 2009).

Hahn et al. (2006) concluded in their study on the role of a bridging organization in wetland conservation in Sweden that an approach to collaboration includes the following key 
characteristics: (1) a flexible 'bridging organization' and 'adhocracy', (2) leadership through trust-building and conflict resolution, (3) generating and communicating ecological knowledge, (4) collaboration and value formation conditional on fixed objectives, and (5) the interplay between formal and informal institutions. These aspects have also been identified to be relevant in connection with the role of NGOs as bridging organizations and their influence on conservation governance on the Tibetan Plateau. In addition, cross-scale linkages and partnerships between different organizational levels were identified as main triggers to collaborative stakeholder partnerships. Conversely, the barriers towards more collaborative conservation governance relate to (1) unequal power relations among different stakeholders, (2) differences in the understanding of how to approach conservation issues, (3) negotiation of a diversity of interests and priorities and (4) issues related to technical and financial resources. In the following, I will give a more detailed discussion of these attributes in relation to the research findings and main objectives.

\subsubsection{Conservation governance arrangements on the Tibetan Plateau}

Scholars have put forward the advantages of decentralizing and democratizing conservation governance and emphasized the benefits of a pluralistic approach that combines non-state practices with state-led regulations in both theory and for the Chinese conservation context (Ostrom, 2005; Fennel et al., 2008, Morton, 2005, McBeath \& Leng, 2006).

Polycentric systems are characterized by more diverse governance structures and there is no central authority dominating the other units of governance (Ostrom, 2005; Pahl-Wostl, 2009). In case of China, however, the state formally has the most central role and decision making power and most conservation-related decisions are made by the central government in Beijing. State conservation efforts suggest that the Chinese government has acknowledged the need for conservation action in the Tibetan Plateau region but conservation on the regional scale is still characterized by large government investments of low conservation effectiveness (Harris, 2008; Yeh, 2009). Interview participants have pointed out that the simplistic and draconian responses to conservation are ill-suited to the ecological and social realities on the ground.

However, while conservation governance on the Tibetan Plateau has long time been dominated by the top-down conservation model, the results show an increasing diversification of conservation approaches. The conservation governance spectrum for the Tibetan Plateau region includes the top-down nature reserve model, co-management agreements between 
government authorities and local resource users within and outside protected areas, community-based natural resource management based on a contract between the government and local communities, and the management of natural resources by traditional resource management institutions. Non-governmental organizations have had a significant influence on this development.

The literature shows that the Chinese government is becoming more tolerant towards the involvement of various forms of societal forces in conservation and NGO have endeavoured to cooperate with government authorities (Lu, 2005; McBeth \& Leng, 2006; Sun \& Zhao, 2007). On the contrary, some scholars described a more hostile political environment for the involvement of civil society in conservation in the Tibetan Plateau region by highlighting the impact of Chinese minority politics and development plans on conservation governance and related disadvantages for local livelihoods and resource management institutions (e.g. Sautman, 2001; Goldstein et al., 2003; Yeh, 2009). As the central government sets the framework for NGOs' freedoms and limitations in operation, it has been questioned how NGOs can act as major agencies of change in a political environment such as that found in China (Morton, 2005; Mc Beath \& Leng, 2006). There is a common agreement in the literature and among NGOs that the current political climate is not suitable for confrontation and differences between cooperation and power sharing between state and non-state actors have to be taken into account.

\subsubsection{NGOs’ role in negotiating conservation governance arrangements}

\section{Network position of NGOs}

The literature argues to understand the impact that bridging organizations have on the governance of natural resources, it is necessary to assess how these organisations span the social gaps among key conservation stakeholders to enable coordinated action. (Berkes, 2009; Bodin \& Crona, 2009). NGOs appear to have a central position in the conservation network on the Tibetan Plateau by sustaining steady linkages to major conservation stakeholders government, local communities, donors and research institutes. However, this central position does not necessarily facilitate communication between all stakeholders nor does it lead to a higher degree of authority among non-state actors. 
Due to differences in power between different parties, building vertical linkages for example between the grassroots level and national government authorities can be a volatile process (Brown, 1991). In addition, the results show that differences in power and influence also exist between NGOs at local, national, and international levels. Even if NGOs attempt to establish cooperative relationships with government authorities at various organizational levels, there exist comparatively weaker ties with the central government than with local and provincial level authorities. However, seeing that the Chinese government is in the process of devolving decision-making authority to regional and local governments (see Chapter II for details), the focus of NGOs on establishing close working ties with these lower levels of government may have the greatest long-term potential in terms of influencing conservation governance arrangements.

According to Brown (1991), bridging organisations might in some cases encourage others to organize to deal with external threats, such as powerful social and political interests. By addressing issues of poverty and economic marginalization, contested perceptions of environmental problems, and the ways in which political and economic systems serve as obstacles to sustainable development, some scholars see social movements as part of Tibetans struggle for cultural self-determination to preserve their national identity (Smith, 2008). During the interviews, the NGOs' representatives have only moderately addressed issues related to the political climate in the Tibetan Plateau region. NGOs do not organize to challenge government authorities but organize to cooperate with them. The representatives affirmed that they do not encourage a large-scale political system change but rather a change in ways of thinking and offer consultation on alternative conservation strategies. They stressed the importance of greater inclusion of local people throughout the conservation process, reconciling conservation and development interests, and improving mutual exchange between the different stakeholder groups.

\section{Approaches towards (adaptive) co-management}

A major question is how to bring stakeholders within the different forms of multi-level governance together and balance the different scales of legitimacy and authority. Berkes (2002) distinguishes between real co-management, with shared management authority, and multi-stakeholder bodies that are often used by government agencies to increase legitimacy and manage conflicts without devolution of power. In the latter case, state officials or NGOs 
present local communities and other resource users with a pre-established plan, leaving little opportunity for negotiating co-management plans (Castro \& Nielsen, 2001). For this reason, scholars argue that bottom-up planning of cross-scale conservation should start at the lowest organizational level (Berkes, 2003; Olsson et al., 2004). Still, not all of the NGOs represented in this research employ a bottom-up approach that lets community choose NGOs and approach them with their project ideas or encourage participation of local communities in the planning process of conservation projects. In addition, NGOs themselves have sometimes little input in national or regional level problem solving processes.

Brechin et al. (2002, p. 45) outline three broad principles for social justice in conservation, which include (1) the right to participate at all levels of the policymaking process as equal partners, (2) the right to self-representation and autonomy, and (3) the right to political, economic, and cultural sovereignty. While the latter two principles are very controversial in view of Chinese minority politics, NGOs are more likely to have influence on the first principle. Comparing the different conservation approaches currently in place in the region, there are different forms and levels of public participation and a diverse involvement of social organisations in regional conservation projects. Despite not having formal authority over other actors, NGOs have been successful in creating institutional arrangements for more collaborative conservation approaches in form of formal agreements between the government and local resource user groups. Still, the individual cases have not yet become institutionalized practices.

It is argued that the international NGO community operates with relatively greater independence from the central government than China-based NGOs, facilitating the progress of democratizing conservation governance through the introduction of western concepts and understandings of participation (Morton, 2005). However, the example of FFI's comanagement project in Sanjiangyuan shows that even though the central government is comparatively tolerant towards international NGOs, problems might show up while dealing with provincial and local government authorities. Though, the literature argues that sometimes a certain degree of conflict is even needed to enter into co-management negotiations (Castro \& Nielsen, 2001; Ostrom, 2005) and the results from my study illustrate that NGOs have the ability to find ways to facilitate communication with both government authorities and communities.

Brown (1991) argues that while the advocacy network of some NGOs has the primary objective of knowledge transfer, it can also be used to influence government policy and 
educate other stakeholders such as donors. Through NGOs influence on information sharing, conservation on the Tibetan Plateau experiences an increasing awareness towards socioecological resilience and to a lesser extent towards traditional resource management institutions. Westley \& Vredenburg (1991) distinguish between egoistic and altruistic bridging organizations. Egoistic organizations tend to be voluntary, self-serving and maintenance oriented, while altruistic organizations are designed or mandated, problem focused, and transformative. NGOs on the Tibetan Plateau act as altruistic organizations so far as they have a focus on the immediate impact of conservation on local livelihoods.

\subsubsection{Key triggers and barriers to collaborative conservation partnerships}

\section{Process-oriented conservation approaches and flexibility}

The way that stakeholders are identified and represented in management regimes is critical to ensure local participation (Castro \& Nielsen, 2001). Local knowledge systems and their embodying communities are not typically homogenous and conflict resolution must be a central part of knowledge integration (Purcell, 2002). In addition, potential differences in culture, ethnicity, social status among participants and the often subtle power dynamics that these differences produce can make negotiation a highly complex undertaking (Brown, 2001; Brechin et al., 2002). Research participants have pointed out the relevance of a diverse sociocultural and economic landscape on the Plateau for conservation. Several interviewees noted government's and donors' struggles with recognizing this diversity and what challenges this creates for transmitting conservation objectives across the network. The literature (Gjertsen \& Barrett 2004; Ostrom, 2007) supports NGOs' argument that there is no blueprint conservation for the region. The research findings illustrate that NGOs have the flexibility and ability to take a more holistic conflict resolution approach to comprehend the socio-cultural, economic and political diversity on the ground and underlying conservation related issues.

\section{Trust building processes}

While investigating social networks, it is also important to understand the ways in which the relatively invisible bonds that bind individual actors together in the absence of legal contracts operate (Kilduff \& Tsai, 2003). Recent research has lead to the important insight that the relationship between stakeholders in conservation is influenced by different factors 
such as levels of trust, transparency, and accountability, benefit and power sharing, or historical grievances (Folke et al., 2005). Trust makes social life predictable, it creates a sense of community, and it makes it easier for people to work together (Shannon, 2000).

Conservation on the Tibetan Plateau is partially characterized by personal, trust-based governance systems that substitute for formal legal ties in and between stakeholder groups. On the one hand, the results showed that personal relationships with government authorities can be useful for getting official permission for projects. On the other hand, the results provide several examples of how NGOs have invested in conflict resolution and trust building mechanisms such as mutual learning, provision of community benefits and a commonsense of partnership to foster collaboration with local communities.

\section{Mutual learning and knowledge partnerships}

Co-management is not only about sharing of power and responsibilities between different groups, but the governance system must also continuously learn and generate experience about ecosystem dynamics and bridging organizations may act as a driving force behind the formation of information networks (Folke et al., 2005; Berkes, 2009). The lack of a common vision of conservation objectives was one of the most significant factors challenging collaborative conservation partnerships. The role of NGOs as facilitators of knowledge exchange between different stakeholders has shown to be significant in the regional conservation context. NGOs see themselves playing a significant role in building and increasing public awareness towards conservation issues with some even having an advisory role at the central government level. Moreover, NGOs deal with scientific ambiguities, a limited knowledge base, and a complex administrative system by providing new information and communication channels through cooperation with national and international conservation experts.

In addition to conservation awareness, the processes responsible for generating community commitments and incentives for conservation are strongly influenced by local conservation capacities and social cohesion (Berkes, 2008). Socio-cultural change in the Tibetan Plateau region has an increasing impact on human-nature relationships and consequently on biodiversity conservation (Goldstein et al., 2003). In terms of weak local institutions, rebuilding the social system is a prerequisite for developing or restoring effective linkages with the ecosystem and for the protection and regulated use of natural resources (Jodha, 2000). 
A crucial challenge for adaptive governance during periods of socio-cultural change is the mobilization of social memory ${ }^{13}$ to link past experiences with present and future policies (Folke et al., 2005). The results illustrated by several project examples NGOs' efforts in the preservation of cultural traditions and traditional resource management institutions. However, it became apparent during the course of most of the interviews that while the inclusion and preservation of traditional culture and resource management institutions is considered to be imperative for conservation, it is challenged by a lack of acknowledgement and support by government and the donor society. In addition, there are significant other problems related to the broader rural development spectrum and the priority of most projects still lies on addressing these issues.

\section{Reconciling conservation and development}

From a social perspective, managing the access to ecosystem services and the decision regarding whose needs are given priority is a fundamental problem (Azqueta \& Delacámara, 2006; Fennel et al., 2008). Both theory and practice have highlighted the importance of providing community benefits in conservation as a prerequisite for conflict resolution and trust-building. The case study of Jamal et al. (2007) on the role of bridging organizations in sustainable development and conservation in Paraguay demonstrated that while local NGOs are valued by the community and local government, tensions can also exist between NGOs and various local stakeholders who perceived the conservation priorities of NGOs cannot be reconciled with local well-being.

NGOs pointed out how important a holistic, process oriented project approach is for the sustainability of biodiversity conservation projects. NGOs' experience from the different projects shows that an acceptance of conservation approaches by local people is often associated with the negotiation of interests and making commitments at different sites. Frequently, conservation goals as long-term communal interests conflict with short-term individual interests and conservation objectives have to be negotiated with livelihood improvements. The reconciliation of conservation and development objectives further demonstrates that bridging organizations can play a critical role in inter-organizational partnerships that enable cooperation on problems that cannot be solved by a single agency and

\footnotetext{
${ }^{13}$ Folke et al. (2005) define "social memory" as the arena in which captured experience with change and successful adaptations, embedded in a deeper level of values, is actualized through community debate and decision-making processes into appropriate strategies for dealing with ongoing change.
} 
even several organizations may share the various roles of a bridging organization in a network (Berkes, 2009).

\section{Creation of cross-sectoral and cross-scale partnerships}

A major theoretical assumption is that inter-organizational relations and collective action are largely driven by the need for power and resources (Cook, 1977). It has been criticized in the literature that the Chinese NGO network is underdeveloped, lacking cooperation between NGOs and in particular between NGOs working in different sectors such as conservation and development (Wu, 2002; McBeth \& Leng, 2006). However, my research results show NGOs rely on networks and there is a notable degree of cooperation between NGOs across different sectors and institutional levels. To solve shared problems, NGOs increasingly expand their project activities to achieve a holistic conservation or development approach through the cooperation with other NGOs working in other sectors.

In their role as bridging organizations, NGOs have the potential to hold together loose associations of organizations that are similar to one another in interests and concerns (Astley \& Fombrun, 1983). By developing tighter and more centralized organizational forms, it is assumed that these coalitions increase organization's potential in influencing regional or national policies (Brown, 1991). The example of CANGO illustrates an important characteristic of a bridging organization. The organization has the necessary leadership and management skills to link the dispersed and diverse constituencies of the network. The organizations experience in acting as a bridge between grassroots activists and conservation experts has built its credibility to promote and support advocacy networks. In addition, CANGO provides its members with communication channels that have potential influence on national policy making.

Apart from the articulation of shared values and visions there are several other factors that influence inter-organizational partnerships (Brown, 1991). The case of the Tibetan Plateau shows that international and national NGOs contributed in supporting local organizations for their own purposes. There can be a difference between a NGO's power and influence. More powerful players such as international conservation NGOs might be influential to the central government but their conservation interests are met with refusal at the community level. By contrast, some stakeholders are more influential on others despite not having a central position in the conservation network. This is for example the case of local grassroots NGOs 
that have a strong linkage to the community level. Several interview partners pointed out the importance of guanxi (informal relationships) and networks at the local for building conservation partnerships. NGOs can be subject to diverse, partially conflicting interests and co-management frequently involves a long process of trust building, negotiation of power and responsibilities to achieve key institutional and organizational changes (Brown, 1991; Olsson et al., 2004). To by-pass the long period of trust-building with local communities or authorities, national and international organizations represented in this research collaborate with local indigenous NGOs. Conversely, even small in capacities, local grassroots organizations can widen their influence through networking, for example by creating partnerships with other NGOs at different hierarchical scales.

Jamal et al. (2007, p.102) demonstrate that building global-local collaborative bridges with grass-roots NGOs can be a useful mechanism for effective local action and change: "[These bridges] act as local conduits for international funding and technology transfer, so that these resources are directed towards preserving threatened ecosystems and local capacity-building activities." The results show that the effectiveness of NGOs to create constructive change and environmental protection is often hampered by factors such as lack of funding, technical training and expertise. Local NGOs profit from the cooperation with NGOs at international and national level through gaining access to higher level government authorities and the national and international network of conservation experts and donors.

While NGOs can play a catalytic role in bringing different stakeholders together, the persistence of newly created networks and the long-term sustainability of improvements generated by projects implemented by external agencies depends on the individual actors in the network (Brown, 1991). The literature argues that local organizations not only help to identify the problems related to conservation on the ground but also have the ability mobilize local resources over the long term (Brown, 2001; Posey, 2002). By bringing in new players from the grassroots level, especially local NGOs, local communities are increasingly given a voice in regional conservation issues. Ostrom (2005) describes the creation of associations of community-governance entities as methods for dealing with threats to sustainability. The representative from Shan Shui has mentioned the organization's effort in creating linkages between different project communities for mutual support and learning. However, the different project examples offer less information about indigenous proactive leadership of dialogue and projects initiated and developed by the community itself. 
However, the problems that are related to a diverse network of actors shows that some of the triggers can at the same time be barriers to more cooperative conservation approaches. Global-local partnerships can empower the local by means of transnational connections or weaken local confidence, resulting in enhanced dependence on those acting in the name of communities (Shih, 2002). Interviewees have mentioned the impact of external priorities for conservation and what consequences this might have for creating partnerships with local stakeholders from civil society. Despite positive developments in the involvement of local interests, conservation approaches are considerably influenced by government and donor interests. In addition, it has to be taken into consideration that NGOs also have their own interests and priorities and try to push them through. Nevertheless, a wider range of donors than in the past has diversified the sources of funding for conservation as well as funding interests. These factors have created functional institutions that not only changed the debates surrounding conservation towards being more focussed on the social aspects of conservation but also enabled a more open discussion of conservation issues and negotiation of interests by addressing a wider spectrum of stakeholders.

\subsection{Research limitations and suggestions for future research}

There are many complex variables that simultaneously affect biodiversity conservation governance and conservation performance. Although my research has provided some significant insights into biodiversity conservation governance in the Tibetan Plateau region, and the role of NGO's as bridging organizations, it leaves considerable room for related studies. This case study was limited to the viewpoint of NGOs. A comparative analysis of different conservation projects based on a limited view of informants of one category has to be aware of a biased position of research participants and does not allow for generalizations. In addition, to get a more in-depth view of the complex network of institutions at play in the region, future research will also need to overcome two key shortcomings of the present study. First of all, interviews were carried out in English which was a foreign language for both the researcher and a large part of the interviewees; second, the research is based on a limited number of research participants.

As there is little research done by independent analysts validating the effects of NGOs efforts on conservation governance in China, it is advisable to investigate and compare the perceptions of other stakeholders such as communities and government authorities on NGOs' 
role in the conservation to validate their role as bridging organizations. Equally, it is worth investigating what potential other state or non-state actors such as research institutes or religious institutions have for acting as bridging organizations. For instance, Khoryug is a conservation network of monasteries in the Himalaya region that aims to develop partnerships with community based organizations and NGOs.

An important question is if there are any noteworthy examples of cooperation on conservation issues between the Chinese government and local communities without NGOs being involved. Thus, additional research is needed that gives attention to local selfgovernance and its relationship to state governance arrangements by investigating the linkages between communities and government authorities at different organizational levels. Research also needs to be done on institutional linkages within and among communities and on the factors that give rise to the alliances and oppositions that shape the dynamics of conservation movements at the grassroots level.

Co-management is not simply the interaction of a unitary state and a homogeneous community (Carlsson \& Berkes, 2005). In my research, I have made a distinction between horizontal and vertical linkages. However, this distinction does completely capture the diversity within the different stakeholders groups and the micro-processes behind negotiating conservation approaches. First of all, my research does not depict the diversity of local resource users and how these are individually affected by conservation decisions. In this research, local voices are only partly represented through the working experience of NGOs. However, there also exist differences in NGOs' interests, capacities and their relationships to individual stakeholder groups.

It has been mentioned by research participants that different opinions and attitudes towards conservation exist within the government and that conflicting interests can be negotiated. Thus, official publications only give a broad idea about the government's attitude towards the issues of interest and are less informative about regional or local variations. For this reason, there is potential for future research on who and what influences conservation decisions at different governmental levels and according to what criteria conservation objectives are constructed.

A major question is in how far the research findings are unique to the Tibetan Plateau region. In this chapter, I compared some of the research findings to case studies from other countries. However, lessons learned from these case studies might not apply to the socio- 
cultural and political context of China. A comparison with conservation developments in different regions in China can provide insight into what lessons have been learned in other regions, and to what extent these can be relevant to conservation issues on the Tibetan Plateau. In addition, the Tibetan Plateau region is recently experiencing significant changes in its economic, social and ecological structures, through both internal developments and external factors. In view of the recent development processes in the region, it has to be questioned if inadequate governance arrangements are still the main problem for conservation, or if rather the new challenges pose a bigger threat for the region's ecosystems. Future research is needed to analyze the role that local institutions (formal and informal) play in causing and confronting to local environmental change.

\subsection{Conclusions}

Specific governance regimes emerge under certain historical, political, cultural, economic and environmental conditions (Pahl-Wostl, 2009). This study offers a glimpse into the multifaceted nature of the networks behind conservation on the Tibetan Plateau. The research findings help to build an understanding of the development of more robust conservation institutions based on co-management that could go a long way for biodiversity conservation in the Tibetan Plateau region. It further gives an insight into the stages government and civil society have completed so far to this development and the challenges encountered on the way.

China is slowly moving from government to governance and the window for NGOs' participation in China's public affairs has opened (Ma, 2007). Given the growth in their number and capacity, NGOs are serving both the state and civil society by acting as a bridge between government agencies, local communities and other organizations across different scales and sectors. NGOs are not only assisting government agencies by acting as policy consultants and service providers, but these organisations also facilitate communication with the international conservation community. My research has also illustrated that biodiversity conservation on the Tibetan Plateau is characterized by an interplay between multiple bridging organizations. Regional and international cooperation has become an important asset for driving the conservation agenda forward. Simultaneously, some environmental NGOs are empowering grassroots groups by sharing their expertise and facilitating access to international support. The research further showed that NGOs have a central function, but not necessarily a central position in the network. An important conservation movement 
accentuates at the grassroots level and its impact on conservation governance has yet to be seen.

While these developments are promising, the extent of improvements that need to be accomplished and the challenges yet to overcome have to be taken into account. These issues have important implications for modelling the impact of NGOs in the improvement of conservation governance arrangements on the Tibetan Plateau. Co-management has increased in scope but is not yet a common practice. Successful partnerships depend on mutually accepted rules to share decision making and to ensure the participation of all partners, including traditionally marginalized groups. The Chinese government has yet to fully recognize the benefits of localizing conservation governance through collaborative partnerships. In addition, more attention needs to be paid to the function of traditional resource management institutions in conservation. The research has shown that NGOs have great potential through their bridging ability and facilitative role in encouraging mutual learning to contribute to a further improvement on regional conservation governance. But rather than seeing power sharing as a starting point as suggested by the literature (Carlsson \& Berkes, 2005), NGOs are more contributing to a subtle transformation of conservation governance regimes.

Despite the many benefits co-management provides, it also has to be questioned if it needed in every single case. The complexity of the issues and the involvement of multiple stakeholders first of all require the creation of a common vision of conservation objectives and integrative approaches. Problems among the different stakeholders are often related to the way of communication and negotiation of interests. Thus, while NGOs are playing a significant role in transforming local conservation regimes, it is equally important to assess what role NGOs are playing in facilitating communication across different organizational levels. In addition, challenges for good governance can only be met in a process-oriented and holistic manner (Folke et al., 2005). While the government still struggles to adopt more adaptive conservation approaches, many NGOs have been be able to build trust and partnerships among key stakeholders by first listening to the needs and interests of their potential partners, and then communicating an attractive vision for their project. These social aspects of conservation are not as easy to quantify as, for example, the numbers of trees protected, but they make a significant contribution to the social dimensions of conservation and sustainable development. 
My case study contributes to the literature by giving an insight into the key triggers and barriers involved in negotiating governance frameworks and developing conservation partnerships. The creation of cross-scale and cross-sectoral partnerships, flexibility, mutual learning and trust-building processes have been identified as key triggers. Unequal power relationships, different understandings of how to approach issues related to conservation and a diversity of partially conflicting interests and priorities create major challenges for collaborative conservation approaches. In addition, my research supports the argument that networks and bridging organizations have an important influence on shaping conservation processes. It further shows that NGOs have a significant potential to act as bridging organizations that coordinate collaborative conservation action. 


\section{Appendix 1: Key triggers and barriers to collaborative conservation approaches}

\begin{tabular}{|c|c|c|}
\hline $\begin{array}{c}\text { Stakeholder } \\
\text { groups }\end{array}$ & Triggers & Barriers \\
\hline $\begin{array}{l}\text { Government } \\
\text { authorities and NGOs }\end{array}$ & $\begin{array}{l}\text { - Identification of local conservation } \\
\text { related issues } \\
\text { - Consultation not confrontation } \\
\text { - Provision of financial resources and } \\
\text { conservation related expertise } \\
\text { - Informal relationships } \\
\text { - Increasing openness to new ideas } \\
\text { - Conservationists at government level } \\
\text { - International reputation of China } \\
\text { - Flexibility in project approaches }\end{array}$ & $\begin{array}{l}\text { - Registration process for NGOs } \\
\text { - Centralized decision making processes } \\
\text { - Complex administrative system } \\
\text { - Little understanding for local traditions } \\
\text { - Power inequalities } \\
\text { - Top down conservation model } \\
\text { - Sensitive political environment } \\
\text { - Government development plans } \\
\text { - Scientific ambiguities } \\
\text { - Level of initial mistrust }\end{array}$ \\
\hline $\begin{array}{l}\text { NGOs and local } \\
\text { communities }\end{array}$ & $\begin{array}{l}\text { - Mutual learning } \\
\text { - Trust building processes } \\
\text { - Comprehensive research approach } \\
\text { - Flexibility in project approaches } \\
\text { - Support of community leadership } \\
\text { - Approaches towards traditional } \\
\text { resource management institutions } \\
\text { - Provision of development alternatives } \\
\text { - Process oriented, holistic approach } \\
\text { - Informal relationships }\end{array}$ & $\begin{array}{l}\text { - Linking communities and government } \\
\text { - institutions at different scales } \\
\text { - Diversity on the ground } \\
\text { - Understandings of the concept of } \\
\text { conservation } \\
\text { - Individual interests } \\
\text { - Changing socio-cultural environment } \\
\text { - Reconciling conservation and } \\
\text { development } \\
\text { - Level of initial mistrust } \\
\text { - Power relationships }\end{array}$ \\
\hline NGOs and NGOs & $\begin{array}{l}\text { - Creation of inter-organizational and } \\
\text { cross-sectoral partnerships } \\
\text { - Links to international } \\
\text { community/grassroots level } \\
\text { - Financial support and expertise } \\
\text { - Local pioneer thinking }\end{array}$ & $\begin{array}{l}\text { - Differences in capacities } \\
\text { - Differences in project priorities } \\
\text { - Cultural differences between northern } \\
\text { and southern NGOs }\end{array}$ \\
\hline $\begin{array}{l}\text { NGOs and donor } \\
\text { society }\end{array}$ & $\begin{array}{l}\text { - Financial support } \\
\text { - Funding related requirements such as } \\
\text { stronger involvement of civil society } \\
\text { in conservation } \\
\text { - Diversification of funding resources } \\
\text { and interests } \\
\text { - Flexibility in project approaches }\end{array}$ & $\begin{array}{l}\text { - Funding priorities } \\
\text { - Little understanding for local traditions } \\
\text { - Levels of mistrust } \\
\text { - Unequal power relationships through } \\
\text { dependency on funding }\end{array}$ \\
\hline Role of NGOs as & - Flexibility and adhocracy & - Differences capacities and resources \\
\hline
\end{tabular}




\begin{tabular}{|l|l|l|}
\hline bridging & - Wide network of funding resources, & - Dominant actors and conservation \\
organizations & information channels & priorities \\
& - Building cross-scale linkages & - Diversity of actors and interests \\
& - Mutual exchange and trust building & - Dependency on funding \\
& - Creation of partnerships & - Own interests and priorities \\
& & - Modern concepts and traditional \\
& & understandings \\
\hline
\end{tabular}




\section{Appendix 2: Interview Schedule}

1. What arrangements for conservation management are currently in practice in your side-specific context?

2. What type of conservation management does your organization advocate for your particular local context?

3. How are key interest groups identified and consulted before and during the implementation process?

4. What role do different stakeholders play in the conservation process? How are conservation-related tasks allocated?

5. What overlapping areas of authority and responsibility are shared between different stakeholder groups?

6. What role do traditional resource management institutions play for the conservation of the ecosystem/natural resource you are focussing on and how, if at all, are these approached under your management plans?

7. What are the main challenges for conservation in the region? And what do you perceive as the main triggers and barriers to the inclusion of traditional institutions in conservation approaches in the region?

8. Where do you see the role of non-state actors such as NGOs in supporting traditional institutions and creating linkages between different levels of conservation governance? 


\section{Appendix 3: Human Ethics Approval, Human Ethics Committee, Victoria University of Wellington}
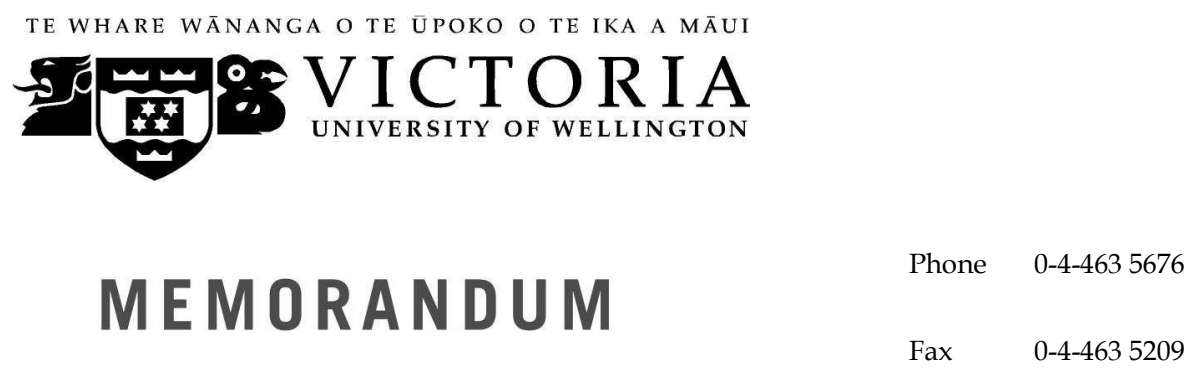

\begin{tabular}{l|l}
\hline TO & Katharina Schlick \\
\hline COPY TO & Michael Gavin \\
\hline FROM & Dr Allison Kirkman, Convener, Human Ethics Committee \\
\hline DATE & 19 July 2010 \\
\hline PAGES & 1 \\
\hline SUBJECT & $\begin{array}{l}\text { Ethics Approval: No 17833 Strengthening local biodiversity } \\
\text { conservation through diverse cross-scale institutional } \\
\text { linkages: traditional resource management institutions and } \\
\text { future pathways for conservation in the Tibetan Plateau. }\end{array}$ \\
\hline
\end{tabular}

Thank you for your applications for ethical approval, which have now been considered by the Standing Committee of the Human Ethics Committee.

Your applications have been approved from the above date and this approval continues until 30 September 2010. If your data collection is not completed by this date you should apply to the Human Ethics Committee for an extension to this approval.

Best wishes with the research.

Allison Kirkman

Convener 


\section{Appendix 4: Participant Information Sheet}

\begin{tabular}{|c|c|c|}
\hline \multicolumn{3}{|c|}{ Participant Information Sheet } \\
\hline \multicolumn{3}{|c|}{$\begin{array}{c}\text { "Strengthening local biodiversity conservation through diverse cross-scale institutional link } \\
\text { Traditional resource management institutions and future pathways for conservation in the } T \\
\text { Plateau" }\end{array}$} \\
\hline$[$ July, 2010] & & \\
\hline $\begin{array}{l}\text { Researcher: } \\
\text { Telephone: } \\
\text { Mobile: } \\
\text { Supervisor: } \\
\text { Email: }\end{array}$ & $\begin{array}{l}\text { Katharina Brigitte Susanne Schlick } \\
\text { 0064-4-4635233-7537 } \\
\text { 0064210327930, 00861376035457 } \\
\text { Dr. Michael Gavin } \\
\text { 0064-4-4635195-5195 } \\
\text { katharinaschlick@gmail.com }\end{array}$ & $\begin{array}{l}\text { School of Geography, Environment and Earth } \\
\text { Sciences (SGEES) } \\
\text { Victoria University of Wellington } \\
\text { PO BOX } 600 \\
\text { Wellington } 6140 \\
\text { (04) } 463-5337\end{array}$ \\
\hline
\end{tabular}

Dear Project Participant,

You are being asked to take part in an interview for my master's thesis at Victoria University of Wellington. The project will investigate the multiple institutional networks behind biodiversity conservation management in the Tibetan plateau region and their impact on the support of traditional resource management institutions in conservation approaches. The key words for this research include: conservation governance, Tibetan Plateau, traditional institutions. The study will answer the following major questions:

1. What governance arrangements for biodiversity conservation are currently in place in the Tibetan Plateau region?

2. What are the triggers and barriers to the inclusion of local traditional resource management institutions into conventional conservation approaches?

3. What role do non-state actors such as NGOs play in facilitating linkages between different institutional levels of conservation governance to support local traditional institutions?

The study will use a variety of qualitative research methods including literature reviews, data analysis, semistructured interviews.

I am inviting you to participate in a semi-structured interview to investigate current developments in conservation governance and institutional networking in the Tibetan Plateau region or in China in general that will not exceed more than 1 hour.

\section{Confidentiality}

At the beginning of the interview, you will be asked in clear, non-technical language whether you wish your identity to remain confidential. I will explain in detail how the results of the research may be used, including the potential for publication in academic journals. The Informed Consent Form (attached) will ask you whether you require confidentiality or not, and how you wish to identify yourself in the research. If you require confidentiality you will be referred to as an official representative of an organisation, or in any other way you request (see \#3. on the Informed Consent Form). 
If you do not require confidentiality, you will be asked to circle the relevant statement (see \#4 on the Informed Consent Form), and only then will your identity be published. No names will appear on the transcripts. A letter-number code will be assigned to each interview respondent, and only this code, or other title specified by you, will appear in the published results (unless confidentiality is waived as described above).

The real names associated with the codes will be kept securely by me, and never made public without the permission of the individual(s) involved.

\section{Storage and Disposal of Data}

Access to the written and electronic material will be restricted to me. All written material will be kept in a locked file, and all electronic material will be password protected. Any interview material or similar will be destroyed and the audio recordings of the interviews will be electronically wiped five years after conclusion of the research.

The results collected will be reported in my thesis, and will be potentially presented in academic journals and conferences. The thesis will be submitted for marking to the School of Geography, Environment and Earth Sciences, Victoria University of Wellington.

\section{Right of Withdrawal}

During the course of the interviews, you will have the right to withdraw from the interview or refuse to answer any question(s) at any time. You may request that the transcript of your interview be destroyed and not used in the study, for any reason. You must inform me of your withdrawal no more than 8 weeks after the date of the interview.

\section{Provision of Feedback}

I will send you a copy of the interview transcript by email for checking its accuracy and to give you the opportunity to make any corrections at any time, prior to final analysis of data. If you would like to make any comments or suggestions to the research in general, please send your feedback by email to katharinaschlick@gmail.com.

\section{Community Access to Research Results}

In order to ensure that the results of this research project are accessible to the participants, a summary of the completed research output will be available. You may request it by circling 'Yes' in Question 7 of the 'Informed Consent Form' (attached), or on later request (by email to katharinaschlick@gmail.com with "Master thesis research results" as subject line). Furthermore, copies of the completed research output will be available from the School of Geography, Environment and Earth Sciences Library, and in the Victoria University of Wellington electronic database of theses.

If you have any other questions about this project, please feel free to ask me now, or contact me, or my supervisor Dr. Michael Gavin later, from the contact details provided on the first page of this sheet.

Thank you for participating!

Sincerely,

\section{Katharina Schlick}




\title{
Appendix 5: Informed Consent Form
}

\author{
Master's Thesis Research Project: \\ "Strengthening local biodiversity conservation through diverse cross-scale institutional linkages: Traditional \\ resource management institutions and future pathways for conservation in the Tibetan Plateau"
}

\section{INFORMED CONSENT FORM}

\begin{tabular}{|ll|l|}
\hline Researcher: & Katharina Brigitte Susanne Schlick & School of Geography, Environment and \\
Telephone: & 0064-4-463-5233-7537 & Earth Sciences (SGEES) \\
Mobile & 0064-210327930 & Victoria University of Wellington \\
Supervisor & Dr. Michael Gavin & PO BOX 600 \\
Tel. & 0064-4-4635195 & Wellington 6140 \\
Email: & katharinaschlick@gmail.com & $(04) 463-5337$ \\
\hline
\end{tabular}

\section{Introduction}

This research for my master thesis in Development Studies at Victory University of Wellington will investigate current developments in biodiversity conservation governance in the Tibetan Plateau region and how traditional resource management institutions are approached in conventional conservation approaches. It will especially focus on the role that non-state actors play in supporting these institutions by facilitating networks between different conservation stakeholders. Approval for this research has been granted by the Victoria University of Wellington Human Ethics Committee. The approval number is 17833. The purpose of this 'Informed Consent Form' is to inform all research participants about the nature of the project and their participation in the research process.

\section{Purpose of the Study}

This study is intended to answer the following main questions:

4. What governance arrangements for biodiversity conservation are currently in place in the Tibetan Plateau region?

5. What are the triggers and barriers to the inclusion of local traditional resource management institutions into conventional conservation approaches?

6. What role do non-state actors such as NGOs play in facilitating linkages between different institutional levels of conservation governance to support local traditional institutions?

Please also see the attached 'Information Sheet'

\section{Research Format}

Qualitative field research will be undertaken in form of semi-structured interviews with research participants who are experts in the field of biodiversity conservation in the Tibetan Plateau region in different locations in China. Participants are being asked to consent to use of information they provide in interviews relating to biodiversity conservation governance and/or traditional resource management institutions in the Tibetan plateau region from them. Questions are not of a personal nature. The duration of the interviews will not exceed one hour. 


\section{Please circle one}

1. I agree to an audio recorded interview with Katharina Schlick

$\mathrm{Y} / \mathrm{N}$

2. I consent to information or opinions that I have given being attributed to me in any reports on this research.

If yes, please answer 3 . If no, please skip to 4 .

$\mathrm{Y} / \mathrm{N}$

3. When opinions and information are attributed to me, I would like to be identified as (please circle one):

a) An 'official' from the organisation I represent, or

b) A letter code, or

c) Other, please specify:

4. I understand that I will have an opportunity to correct the transcript of the interview before publication.

$\mathrm{Y} / \mathrm{N}$

5. I understand that the data I provide will not be used for any other purpose than that described in the 'Information Sheet', and that data will not be released to others without my written consent.

$$
\mathrm{Y} / \mathrm{N}
$$

6. I would like to receive a summary of the results of this research when it is completed.

$$
\mathrm{Y} / \mathrm{N}
$$

If yes, My address is:

My email is:

In case I like to withdraw from the research project and do not wish the transcript of my interview to be published, I must inform the researcher of my withdrawal no more than 8 weeks after the date of the interview.

I agree to take part in this research

Participant:

Name:

Date:

Signature: 


\section{References}

Agrawal, A., Gibson, C. B. (1999). Enchantment and disenchantment: The role of community in natural resource conservation. World Development, 27 (4), 629-649.

Armitage, D. (2005). Adaptive capacity and community-based natural resource management. Environmental Management, 35 (6), 703-715.

Armitage, D., Marschke, M., Plummer, R. (2008). Adaptive co-management and the paradox of learning. Global Environmental Change, 1, 86-98.

Astley, W.E., Fombrun, C.T. (1983). Collective strategy: social ecology of organizational environments. Academy of Management Review, 8 (4), 576-587.

Azqueta, D., Delacámara, G. (2006). Ethics, economics and environmental management. Ecological Economics, 56 (4), 524-533.

Banks, T., Richard, C., Li, P., Yan, Z. (2003). Community Based Grasslands Management in Western China. Mountain Research and Development, 23 (2), 132-140.

Berger, J. (2010). Impressions: How special is Tibet's Changtang? China's Tibet, 21 (3), 6470.

Berkes, F., Folke C. (1992). A systems perspective on the interrelations between natural, human-made and cultural capital. Ecological Economics, 5, 1-8.

Berkes, F., Colding, J., Folke, C. (2000). Rediscovery of traditional ecological knowledge as adaptive management. Ecological Applications, 10 (5), 1251-1262.

Berkes, F., Folke, C. (Eds.) (2000). Linking social and ecological systems: Management practices and social mechanisms for building resilience. Cambridge: Cambridge University Press.

Berkes, F. (2002). Cross-Scale institutional linkages: Perspectives from the bottom up. In E.T. Ostrom, T. Dietz, N. Dolšak, P.C. Stern, S. Stonich, and E. Weber (Eds.), The Drama of the Commons, pp. 293-321. Washington, D.C: National Academy Press.

Berkes, F., Colding, J., Folke, C. (Eds.) (2003). Navigating social-ecological systems: Building resilience for complexity and change. Cambridge: Cambridge University Press.

Berkes, F. (2004). Rethinking community-based conservation. Conservation Biology, 18 (3). 621-630.

Berkes, F., Turner, N.J. (2006). Knowledge, learning and the evolution of conservation practice for social-ecological system resilience. Human Ecology, 34 (4), 479-494.

Berkes, F. (2008). Sacred Ecology, $2^{\text {nd }}$ Edition. London: Routledge.

Berkes, F. (2009). Evolution of co-management: Role of knowledge generation, bridging organizations and social learning. Journal of Environmental Management 90, 1692-1702. 
Bhalla, A.S., Qiu, S. (2006). Poverty and inequality among Chinese minorities. New York: Routledge.

Bodin, Ö., Crona, B.I. (2009). The role of social networks in natural resource governance: What relational patterns make a difference? Global Environmental Change (19), 366-374.

Brechin, S.R., Wilshusen, P.R., Fortwangler, C.L., and P.C. West (2002). Beyond the square wheel: Toward a more comprehensive understanding of biodiversity conservation as social and political process. Society and Natural Resources, 15, 41- 64.

Brosius, J.P., Lowenhaupt Tsing, A., Zerner, A. (2005). Communities and conservation: Histories and politics of community-based natural resource management. Lanham: Alta Mira Press.

Brown, D. (1991). Bridging organizations and sustainable development. Human Relations, 44 (8), 807-831.

Burt, R.S. (1992). Structural Holes: The social structure of competition. Cambridge, MA: Harvard University Press.

Burt, R.S. (2004). Structural holes and good ideas. American Journal of Sociology (110), 349-399.

Cai, R. (2008). Is wildlife adapting to the Qinghai-Tibet railway? Retrieved 22 February, 2011, from: http://www.chinadialogue.net/article/show/single/en/2080.

Carlsson, L., Berkes, F. (2005). Co-management: Concepts and ecological implications. Journal of Environmental Management 75, 65-76.

Carter, N.T., Mol, A.P.J. (Eds.) (2007). Environmental governance in China. Oxon: Routledge.

Castro, A.O., Nielsen, E. (2001). Indigenous people and co-management: implications for conflict management. Environmental Science and Policy Volume, 4, (4/5), 229-239.

Clarke, G. E. (Ed.) (1998). Development, society, and environment in Tibet: Papers presented at a panel of the 7th Seminar of the International Association for Tibetan Studies, Graz 1995. Wien: Verlag der Österreichischen Akademie der Wissenschaften.

Coggins, C. (2003). The tiger and the pangolin: Nature, culture and conservation in China. The University of Hawai'i Press.

Common, M. \& Stagl, S. (2005). Ecological Economics: An Introduction. Cambridge University Press.

Cook, K. (1977). Exchange in power and network in inter-organizational relations.

Sociological Quarterly, 18, 62-82.

Cook, K. (2003). Trust in Society. New York: Sage. 
Escobar, A. (1998). Whose knowledge, whose nature? Biodiversity, conservation, and the political ecology of social movements. Journal of Political Ecology, 5, 53-82.

Fabricius, C., Folke, C., Cundill, G., Schultz, L. (2007). Powerless Spectators, Coping Actors, and Adaptive Co-managers: A Synthesis of the Role of Communities in Ecosystem Management. Ecology and Society 12(1): 29. Retrieved 22 March, 2010, from: http://www.ecologyandsociety.org/vol12/iss1/art29/.

Fauna \& Flora International (FFI) (2004). Co-management Plan for Biodiversity Conservation and Community Livelihoods in the Suojia Area: Including results of surveys and analysis of local biodiversity and community socio-economics.

Beijing: Flora \& Fauna International.

Fennel, D. Plummer, R., Marschke, M. (2008). Is adaptive co-management ethical? Journal of Environmental Management 88, 62-75.

Fisher, R. et al. (2008). Linking conservation and poverty reduction: Landscapes, people and power. London: Earthscan.

Fleming, R.L., Tsering, D., Lu, W. (2007). Across the Tibetan Plateau: Ecosystems, wildlife and conservation. New York: W. W. Norton.

Foggin, M., Smith, A. T. (2000). Rangeland utilization and biodiversity on the Alpine grasslands of Qinghai Province, PRC. In L. Zhi and J. Springer (Eds.), Tibet's biodiversity conservation and management, pp. 120-130. Beijing: China Forestry Publishing House.

Foggin, J.M. (2005). Highland Encounters: Building new partnerships for conservation and sustainable development in the Yangtze River headwaters, heart of the Tibetan Plateau. In J. Velasquez, M. Yashiro, S. Yoshimura and I. Ono (Eds.), Innovative Communities: Peoplecentred Approaches to Environmental Management in the Asia-Pacific Region. Tokyo, Japan: United Nations University (UNU) Press. 360 pp. Retrieved 15 February, 2010, from: http://www.plateauperspectives.org/downloads/Highland\%20Encounters.pdf.

Foggin, J.M. (2008). Depopulating the Tibetan grasslands: National policies and perspectives for the future of Tibetan herders in Qinghai Province, China. Mountain Research and Development, 28 (1), 26 -31.

Folke, C., Carpenter. S., Walker, B., Scheffer, M., Elmqqvist, T., Gunderson, L. and C.S. Holling (2004). Regime shifts, resilience, and biodiversity in ecosystem management. Annual Review of Ecological Evolutionary Systems, 35, 557-581.

Folke, C., Hahn, T., Olsson, P., Norberg, J., 2005. Adaptive governance of socialecological systems. Annual Review of Environment and Resources 30 (8), 1-8.

Foreign Culture Exchange Association of Tibet Autonomous Region (2007). Population of the Tibet Autonomous Region. Beijing: China Intercontinental Press. 
Ginat, J., Khazanov, A. M. (1998). Changing nomads in a changing world. Brighton: Sussex Academic Press.

Gjertsen, H., Barrett, C.B. (2004). Context-dependent biodiversity conservation management regimes: Theory and simulation. Land economics, 80 (3), 321-339.

Goldstein, M. C., Jiao, B., Beall, C. M., Tsering, P. (2003). Development and change in rural Tibet: Problems and adaptations. Asian Survey, 43 (5), 758-779.

Goodland, R. (2008). Conservation in sensitive biodiversity areas. In L. Rockwood, R. Stewart and T. Dietz (Eds.), Foundations of environmental sustainability: The coevolution of science and policy, pp.67-80. New York: Oxford University Press.

Granovetter, M. (1973). The strength of weak ties. American Journal of Sociology, 78, 13601380 .

Gransow, B. (2009). Institutional change in China's anti-poverty policy. In Heberer, T., Schubert, G. (Eds.), Regime legitimacy in contemporary China: institutional change and stability, pp. 245-267. Oxon: Routledge.

Gruschke, A. (2006). Nomaden ohne Weide? Eurasisches Magazin, 5-6. Retrieved 20 February, 2010, from:

http://www.eurasischesmagazin.de/artikel/?artikelID=20060510\&marker=nomaden\%20ohne \%20weide.

Gunderson, L.H., Allen, C.R., Holling, C.S. (Eds.) (2010). Foundations of ecological resilience. Washington, D.C.: Island Press.

Hahn, T., Olsson, P., Folke, C., Johansson, K., 2006. Trust-building, knowledge generation and organizational innovations: the role of a bridging organization for adaptive comanagement of a wetland landscape around Kristianstad, Sweden. Human Ecology, 34, $573-592$.

Harris, R.B. (2008). Wildlife conservation in China: preserving the habitat of China's Wild West. Armonk, NY: M.E. Sharpe.

Ho, P., Eyferth, J., Vermeer, E.B. (2004). Rural development in transitional China: The new agriculture. London: Frank Cass Publishers.

Holling, C.S., Berkes, F., Folke, C. (2000). Science, sustainability and resource management. In F. Berkes, C. Folke (Eds.), Linking social and ecological systems: Management practices and social mechanisms for building resilience, pp. 342-362. Cambridge: Cambridge University Press.

Jamal, T., Kreuter, U., Yanosky, P. (2007). Bridging organisations for sustainable development and conservation: a Paraguayan case. International Journal of Tourism Policy, 1 (2), $93-110$. 
Janssen, M.A., Walker, B.H., Langridge, J., Abel, N. (2000). An adaptive agent model for analysing co-evolution of management and policies in a complex rangeland system.

Ecological Modelling, 13, 249-268.

Jodha, N. S. (2000). Reviving the social system-ecosystem links in the Himalayas. In F. Berkes, C. Folke, C. (Eds.). Linking social and ecological systems: Management practices and social mechanisms for building resilience, pp.285-310. Cambridge: Cambridge University Press.

Kalland, A. (2000). Indigenous knowledge: Prospects and limitations. In R. Ellen, P. Parkes, A. Bicker (Eds.), Indigenous environmental knowledge and its transformation. Critical anthropological perspectives, pp.319-335. London: Routledge.

Khazanov, A. M. (1998). Pastoralists in the contemporary world: The problem of survival, Ginat, J., Khazanov, A. M. (Eds.). Changing nomads in a changing world, pp.7-23. Brighton: Sussex Academic Press.

Khazanov, A. M., Wink, A. (Eds.) (2001). Nomads in the sedentary world. Richmond, Surrey: Curzon.

Khazanov, A. M. (2001). Nomads in the history of the sedentary world. In A.M. Khazanov, A. Wink (Eds.), Nomads in the sedentary world, pp.1-23. Richmond, Surrey: Curzon.

Kilduff, M., Tsai, W. (2003). Social networks and organizations. London: Sage.

Klieger, C. (2007). The people of Tibet. In B. Brower, B.R. Johnston (Eds.). Disappearing Peoples? Indigenous groups and ethnic minorities in South and Central Asia, pp.219-241. Walnut Creek, CA: Left Coast Press.

Kolas, A., Thowsen, M. (2005). On the margins of Tibet: Cultural survival on the SinoTibetan frontier. Seattle, WA: University of Washington Press.

Kooiman, J. (2003). Governing as governance. London: Sage.

Lai, H., China's Western Development Program: Its rationale, implementation, and prospects, Modern China, 28 (4), 432-466.

Lancaster, W., Lancaster, F. (1998). Who are these Nomads? What do they do? Continuous change or changing continuities? In J. Ginat, J., A.M. Khazanov (Eds.). Changing nomads in a changing world, pp. 24-37. Brighton: Sussex Academic Press.

Leal, P.A. (2007). Participation: the ascendancy of a buzzword in the neo-liberal era. Development in Practice, 17 (4-5), 539-548.

Liu, J. Dietz, T., Carpenter, S.R., Alberti, M., Folke, C., Moran, E. Pell, A.N., Deadman, P., Kratz, T., Lubchenco, J., Ostrom, E., Ouyang, Z., Provencher, W., Redman, C.L., Schneider, S.H.,W.W. Taylor (2007). Complexity of coupled human and natural systems. Science, 317, $1513-1516$. 
Lo, W., Tang, C. (2007). Institutional reform, economic changes, and local environmental management in China: the case of Guangdong Province. In N.T. Carter, A.P.J. Mol (Eds.), Environmental governance in China, pp. 42-62. Oxon: Routledge.

Low, B.S., et al., (2003). Redundancy and diversity in governing and managing commonpool resources. In F. Berkes, J. Colding, C. Folke (Eds.), Navigating Social-Ecological Systems: Building Resilience for Complexity and Change, pp.83-114. Cambridge: Cambridge University Press.

Lu, D., Neilson, W.A. (Eds.) (2004). China's West Region Development - Domestic Strategies and Global Implications. Singapur: World Scientific Publishing Company.

Lu, Y. (2005). Environmental civil society and governance in China. Chatham House Asia Programme Briefing Paper 05/04. Retrieved 22 January 2011 from:

http://www.chathamhouse.org.uk/files/10225_300107workshop.pdf.

Ma, S.K. (2007). What Can We Learn from Recent Developments in China's Public Administration? Public Administration Review, 67, 1088-1091.

Ma, X., Ortolano, L. (2000). Environmental regulation in China: Institutions, enforcement, and compliance. Lanham: Rownman \& Littlefield Publishers.

Martens, S. (2007). Public participation with Chinese characteristics: Citizen consumers in China's environmental management. In N.T. Carter, A.P. Mol (Eds.), Environmental governance in China, pp.63-82. Oxon: Routledge.

McBeath, G.A., Tse-Kang Leng (2006). Governance of biodiversity conservation in China and Taiwan. Northampton M.A.: Edgar Elgar Publishing, Inc.

McGinnis, M. (2000). Polycentric Governance and Development. Ann Arbor, MI: University of Michigan Press.

Miller, D. (1998). Nomads of the Tibetan Plateau Rangelands in Western China, Part One: Pastoral History. Rangelands, 20(6), 24-29.

Miller, D. (2002). The importance of China's nomads. Rangelands, 24 (1), pp. 22-24.

Mol, A. P.J., Carter, N.T. (2007). China's environmental governance in transition. In N.T. Carter, A.P. Mol (Eds.), Environmental governance in China, pp. 1-22. Oxon: Routledge.

Morton, K. (2005). International aid and China's environment: Taming the yellow dragon. New York: Routledge.

Morton, K. (2008). China and environmental security in the age of consequences. Asia Pacific Review, 15 (2), 52-67.

Naeem, S., Bunker, D.E., Hector, A., Loreau, M., Perrings, C. (Eds.) (2009). Biodiversity, ecosystem functioning, \& human wellbeing: An ecological and economic perspective. Oxford: Oxford University Press. 
Noble, B.F. (2000). Institutional criteria for co-management. Marine Policy, 24, 69-77.

Norbu, N.(1997). Journey among the Tibetan nomads: An account of a remote civilization. Dharamsala: Library of Tibetan Works \& Archives.

Oberheitmann, A. (2009) Environmental policy reform in China. In T. Heberer, T., G. Schubert (Eds.). Regime legitimacy in contemporary China: institutional change and stability, pp. 268-296. Oxon: Routledge.

Olsson, P., Folke, C., Berkes, F. (2004). Adaptive co-management for building resilience in social-ecological systems. Environmental Management, 34 (1), 75-90.

O'Riordan, T., Stoll-Kleemann, S. (Eds.) (2002). Biodiversity, sustainability and human communities: Protecting beyond the protected. Cambridge: Cambridge University Press.

Ostrom, E. (1990). Governing the Commons: The evolution of institutions for collective Action. Cambridge University Press.

Ostrom, E. (2005). Understanding institutional diversity. Princeton, New Jersey: Princeton University Press.

Ostrom, E. (2007). A diagnostic approach for going beyond panaceas. Proceedings of the National Academy of Sciences (PNAS) 104 (39), 15181-15187.

Pahl-Wostl, C. (2009). A conceptual framework for analysing adaptive capacity and multilevel learning processes in resource governance regimes. Global Environmental Change, 19, 354-365.

Parfitt, T. (2004). The ambiguity of participation: A qualified defence of participatory development. Third World Quarterly, 25 (3), 537-556.

Pendeba Society (2010). 珠穆朗玛峰国家自然保护区 潘得巴协会 简报 (Report of the Pendeba Society of the Qomolangma National Nature Preserve).

Plummer, R., Fitzgibbon, J. (2004). Co-Management of natural resources: A proposed framework. Environmental Management, 33, (6), 876-885.

Plummer, R., Armitage, D. (2007). A resilience-based framework for evaluating adaptive comanagement: Linking ecology, economics and society in a complex world. Ecological Economics, 61, 62-74.

Prager, K. (2010). Local and Regional Partnerships in Natural Resource Management: The Challenge of Bridging Institutional Levels. Environmental Management, 46 (5), 711-724.

Pretty, J., Ward, H. (2001). Social capital and the environment. World Development, 29, 209227.

Rammel. C., Stagl, S., Wilfing, H. (2007). Managing complex adaptive systems - A coevolutionary perspective on natural resource management. Ecological Economics 63, 9-21. 
Rao, K. S., Semwal, R. L., Nautiyal, S., Sen, K.K., Singh, K., Chandrasekhar, K., Saxena, K. G. (2003). Indigenous ecological knowledge, biodiversity and sustainable development in the central Himalayas. Tropical Ecology, 44 (1), 93-111.

Rhodes, R.A.W., (1997). Understanding governance: Policy networks, reflexivity and accountability. Buckingham: Open University Press.

Richard, C. Yan, Z., Du, G. (2006). The paradox of the individual household responsibility system in the grasslands of the Tibetan plateau, China. In D.J. Bedunah, E.D. McArthur and M. Fernandez-Gimenez (Eds.), Rangelands of Central Asia, pp. 83-91. USDA Rocky Mountains Research Station Proceedings RMRS-P-39.

Rootes, C. (2008). Acting locally: the character, contexts and significance of local environmental mobilisations. In C. Rootes (Ed.) (2008), Acting locally: Local environmental mobilizations and campaigns, pp. 2-21. Oxon: Routledge.

Rossabi, M. (Ed.) (2004). Governing China's multiethnic frontiers. Seattle: University of Washington Press.

Salzman, P.C. (Ed.) (1980). When nomads settle: Process of sedentarization as adaption and response. New York: Bergin Publishers.

Sausmikat, N. (2009). Civil society dilemmas in dealing with China. EU-China Civil Society Forum. Retrieved 12 February, 2011, from:

http://www.eu-china.net/web/cms/upload/pdf/materialien/eu-

china_2009_hintergrund_04.pdf.

Sautman, B., Eng, I. (2001). Tibet: Development for whom? China Information, 15 (20), 2074.

Sautman, B. (2002). Resolving the Tibet question: problems and prospects. Journal of Contemporary China, 11 (30), 77-107.

Scholz, J. T., Wang. C. (2006). Cooptation or transformation? Local policy networks and federal regulatory enforcement. American Journal of Political Science, 50(1), 81-97.

Scott, R.W. (2001). Institutions and Organizations, $2^{\text {nd }}$ Edition. Thousand Oaks, CA: Sage Publications.

Shackeroff, J.M., Campell, L.M. (2007). Traditional ecological knowledge in conservation research: Problems and prospects for their constructive engagement. Conservation and Society, 5 (3), 343-360.

Shannon M.A. 1990. Building trust: the formation of a social contract. In R.G. Lee, D.R. Field, W.R. Burch jr. (Eds) Community and Forestry: Continuities in the Sociology of Natural Resources, pp. 229-40. Boulder, CO: Westview.

Shan Shui “Through their eyes project” (xiangcun zhi yan 乡村之眼). Retrieved 12 February, 2011, from: http://www.shanshui.org/Landscape/Project/ngs/. 
Shapiro, J. (2001). Mao's war against nature: Politics and the environment in revolutionary China. Cambridge: Cambridge University Press.

Sheehy, D. P., Miller, D, Johnson, D. A. (2006). Transformation of traditional livestock systems on the Tibetan steppe. Sécheresse, 17 (1-2), 142-151.

Sillitoe, Paul, Marzano M. (2009). The future of indigenous knowledge research in development. Futures, 41, 13-23.

Simmons, L. (2002). Tibetans: Moving Forward, Holding On. National Geographic.

Retrieved 17 March, 2010, from:

http://ngm.nationalgeographic.com/features/world/asia/tibet/tibetans-text?source=ge-asia.

Silverman, D. (2010). Doing qualitative research, $3^{\text {rd }}$ Edition. London: Sage Publications.

State Council of the People's Republic of China (1998). Regulations on the Registration and Administration of Social Organisations. Beijing: State Council of the People's Republic of China. Translation retrieved 12 December, 2010, from:

http://www.chinadevelopmentbrief.com/node/298.

State Council Information Office of the People's Republic of China (2003). White Paper on Ecological Improvement and Environmental Protection in Tibet. Beijing: State Council Information Office of the People's Republic of China. Retrieved 18 October, 2010, from: http://english.peopledaily.com.cn/whitepaper/tbpaper/tb.html.

State Forestry Agency Department of Wildlife Conservation (2002). Guidelines of Community-based Natural Resources Co-management in Nature Reserves. Beijing: State Forestry Press.

Steinberg, P. (2001). Environmental leadership in developing countries: Transnational relations and biodiversity policy in Costa Rica and Bolivia. Cambridge: The MIT Press.

Strauss, A., Corbin, J. (1998). Basics of qualitative research: techniques and procedures for developing grounded theory, $2^{\text {nd }}$ Edition. Thousand Oaks, CA: Sage Publications.

Studley, J. (2007). Hearing a Different Drummer: A new paradigm for the "keepers of the forest". London: International Institute for Environment and Development. Retrieved 5 April, 2010, from: http://www.iied.org/pubs/pdfs/14541IIED.pdf.

Sun, Y., Zhao, D. (2007). Multifaceted state and fragmented society: Dynamics of environmental movement in China. In D.L.Yang (Eds.), Discontended miracle: Growth, conflict, and institutional adaptations in China, pp.111-160. New Jersey: World Scientific.

Tang, R., Gavin, M. (2010). Traditional ecological knowledge informing resource management: Saxoul conservation in Inner Mongolia, China. Society \& Natural Resources, $23(3), 193-206$. 
Tibetan \& Himalayan Library, Tibet and adjoining areas terrain map. Retrieved 10 February, 2011, from: http://www.thlib.org/places/maps/collections/show.php?id=275.

Tibetan \& Himalayan Library, Tibet autonomous prefectures. Retrieved 10 February, 2011, from: http://www.thlib.org/places/maps/collections/show.php?id=125.

Tsomo, T. (2010). Vanishing nomads of Tibet. Border Affairs. 12 (1). Retrieved 1 March, 2011, from: http://tibetspeak.wordpress.com/2010/10/13/vanishing-nomads-of-tibet/.

Tyler, S.R. (2006). Comanagement of natural resources: Local learning for poverty reduction. Ottawa: International Development Research Center.

Vernooy, R., Qiu, S., Xu, J. (2006). The power of participatory monitoring and evaluation: Insights from south-west China. Development in Practice, 16 (5), 400-411.

Waddel, E., Connell, J. (2007). Between global and local: the contest for development. In J. Connell, E. Waddell (Eds.), Environment, development and change in rural Asia-Pacific, pp.1-15. London: Routledge.

Westley, F., Vredenburg, H. (1991) 'Strategic bridging: the collaboration between environmentalist and business in the marketing of green products', Journal of Applied Behavioral Science, 27 (10), 65-90.

Wikimedia, China relief map. Retrieved 2 May, 2010, from:

http://commons.wikimedia.org/wiki/File:ChinaGeography.png.

Wikimedia, Sanjiangyuan National Nature Reserve map. Retrieved 21 March, 2010, from: http://commons.wikimedia.org/wiki/File:SNNR_Map.jpg.

Wilshusen, P.,Murguía, R. (2003). "Scaling up from the grassroots: NGO networks and the challenges of organizational maintenance in Mexico's Yucatán Peninsula. In Brechin, Wilshusen, Fortwangler and West, (Eds). Contested Nature: Toward International Biodiversity with Social Justice in the 21st Century, pp. 195-215. SUNY Press.

Wu, N. (2000). Man's impact on biodiversity in Tibetan nomadic societies in Western Sichuan. In L. Zhi, J. Springer (Eds.), Tibet's biodiversity conservation and management, pp.113-119. Beijing: China Forestry Publishing House.

Wu, F. (2002). New partners or old brothers? GONGOs in transnational environmental advocacy in China. China Environment Series, 5, 45-58.

Zhang, X., Baum, R. (2004). Civil society and the anatomy of a rural NGO. The China Journal, 52, 97-107.

Xu, J., Ma, E., Tashi, D., Fu, Y., Lu, Z., Melick, D. (2006). Integrating sacred knowledge for conservation: Cultures and landscapes in south-west China. Ecology and Society, 10 (2), 7.

Xu, J., Melick, D. (2007). Towards community driven conservation in southwest China: reconciling state and local perceptions. World Agroforestry Centre Working Paper Number 52. Beijing: ICRAF. 
Yang, S., Bleisch, W.V., and D. Mallon (2006). Community participation in planning and action for conservation on the Qinghai-Tibet Plateau. In McNeely, J.A., T. M. McCarthy, A. Smith, L. Olsvig-Whittaker, and E.D. Wikramanayake (Eds). Conservation Biology in Asia. Kathmandu: Society for Conservation Biology Asia Section and Resources. Retrieved 12 January, 2010, from:

http://www.conbio.org/sections/Asia/docs/Chapter\%208_Shao_Qinghai-Tibet.pdf .

Yeh, E. T. (2003). Tibetan range wars: Spatial politics and authority on the grasslands of Amdo. Development and Change, 34(3), 499-523.

Yeh, E.T. (2005). Green governmentality and pastoralism in Western China: 'Converting pastures to grasslands'. Nomadic Peoples, 9 (1-2), 9-30.

Yeh, E. T. (2009). From wasteland to wetland? Nature and nation in China's Tibet. Environmental History, 14 (1), 103-137.

Young, O., (2008). Building regimes for socio-ecological systems: Institutional diagnostics. In O.Young, L. King, H. Schroeder (Eds.), Institutions and Environmental Change, pp.115144. Cambridge, MA: MIT Press.

Zhang, B., Chen, X, Li, B., Yao, Y. (2002). Biodiversity and conservation in the Tibetan Plateau. Journal of Geographical Sciences, 12 (2), 135-143.

Zhang, M., Wang, S. (2004). Co-management: transformation of community affair model in Chinese nature reserves. Journal of Forestry Research, 15 (4), 313-318.

Zheng, Y., Qian Y. (2004). Eco-Environmental Protection and Poverty-Alleviation in West China Development. In D. Lu, W. Neilson (Eds.), China's West Region Development Domestic Strategies and Global Implications, pp. 27-40. Singapur: World Scientific Publishing Company.

Zhi, L., Springer, J. (2000). Tibet's biodiversity conservation and management. Beijing: China Forestry Publishing House. 